Universidade de São Paulo

Instituto de Física

\title{
Efeito Rashba em Isolantes Topológicos
}

\author{
Oscar Andres Babilonia Pérez
}

Orientador: Prof. Dr. Adalberto Fazzio

Dissertação apresentada ao Instituto de

Física da Universidade de São Paulo para obtenção do título de Mestre em Ciências

Banca Examinadora:

Prof. Dr. Adalberto Fazzio (IF-USP)

Prof. Dr. Koiti Araki (IQ-USP)

Prof. Dr. Gustavo Martini Dalpian (UFABC)

São Paulo

2016 
FICHA CATALOGRÁFICA

Preparada pelo Serviço de Biblioteca e Informação do Instituto de Física da Universidade de São Paulo

Babilonia Pérez, Oscar Andres

Efeito Rashba em isolantes topológicos. São Paulo, 2016.

Dissertação (Mestrado) - Universidade de São Paulo. Instituto de Física. Depto. de Física dos Materiais e Mecânica.

Orientador: Prof. Dr. Adalberto Fazzio

Área de Concentração: Física da Matéria Condensada.

Unitermos: 1. Propriedades dos sólidos; 2. Cálculo de primeiros princípios; 3. Teoria do funcional da densidade; 4. Efeito Rashba; 5. Isolantes topológicos. 
University of São Paulo

Institute of Physics

\title{
Rashba effect in Topological Insulators
}

\author{
Oscar Andres Babilonia Pérez
}

Advisor: PhD Adalberto Fazzio

\begin{abstract}
Dissertation submitted to Institute of Physics of the University of São Paulo to obtain the title of Master in Sciences
\end{abstract}

\section{Examining Committee:}

Prof. Dr. Adalberto Fazzio (IF-USP)

Prof. Dr. Koiti Araki (IQ-USP)

Prof. Dr. Gustavo Martini Dalpian (UFABC)

\section{São Paulo}





\section{Agradecimentos}

Primeiramente agradeço a Deus por colocar sempre as probabilidades ao meu favor.

Aos meus pais, Damaris e Marcos por ser sempre uma fonte de força e motivação.

Aos meus irmãos, Javier e Marcos pelo apoio moral, econômico, e a confiança incondicional.

Ao meu orientador Adalberto Fazzio por me permitir trabalhar no grupo SAMPA, pelas discussões acadêmicas e os bons conselhos.

Ao Carlos Mera que foi quase um coorientador, pela amizade e exemplo.

Aos colegas do grupo SAMPA, especialmente ao Alexsandro e Leonardo que foram irmãos.

Aos professores do grupo SAMPA, José Roque Da Silva quem me ajudou com a carta de recomendação para conseguir minha bolsa de estudo e ao professor Caetano Miranda pela amizade e por me permitir ser seu orientando na minha próxima etapa acadêmica.

A minha grande amiga Olivia, por me ajudar nas correções do português.

A Karla pelas correções, pelo carinho e os bons papos.

Aos meus compatriotas, Alex, Shirley, Carlos Mario, Anuar e Eder pela amizade e as batalhas lutadas em tempos difíceis. Especialmente ao Eder quem me ajudou grandemente na minha chegada ao Brasil.

À agencia CNPq pelo financiamento. 
"The science of today is the technology of tomorrow"

\section{-Edward Teller}




\section{RESUMO}

Neste trabalho de mestrado apresentamos um estudo sobre a manifestação do efeito Rashba em isolantes topológicos na ausência de simetria de inversão estrutural. Os cálculos das propriedades atomísticas, energéticas e as estruturas eletrônicas são abordados através de métodos de primeiros princípios baseados na teoria do funcional da densidade. E seus resultados foram utilizados para o desenvolvimento de hamiltoniana efetiva baseado no modelo de Zhang.

Realizamos o estudo de dois sistemas:

1) $\mathrm{Bi}_{2} \mathrm{Se}_{3}$ com átomos de $\mathrm{Sn}$ depositados na superfície: Este sistema pode ser entendido através da manifestação do efeito Rashba sobre um isolante topológico dada a quebra de simetria de inversão estrutural. Para um sítio de deposição específico, os átomos de Sn causam uma reconstrução da superfície e um terceiro cone de Dirac é observado na estrutura eletrônica. Este terceiro cone é não localizado na superfície e pode ser entendido como a manifestação do efeito Rashba.

2) PbBiI: Reportado aqui como um novo isolante topológico $2 \mathrm{D}$ com efeito Rashba. Descobrimos este sistema por um estudo sistemático sobre uma família de materiais formados por átomos tipo IV, V, e VII, cuja estrutura cristalina é hexagonal e não centrossimétrica. Mostramos que o PbBiI possui: i) Estabilidade mecânica, ii) Spin-splitting Rashba de $60 \mathrm{meV}$, iii) um gap de energia não trivial de $0.14 \mathrm{eV}$, iv) retroespalhamento proibido entre os estados de borda e v) retroespalhamento proibido entre os estados do bulk no entorno do nível de Fermi. Estas propriedades fazem do PbBiI um candidato para construção de dispositivos de spintrônica que atenua a perda de energia.

Palavras chave: Propriedades dos sólidos, Cálculo de Primeiros Princípios, Teoria do Funcional da Densidade, Efeito Rashba, Isolantes topológicos. 


\section{Abstract}

In this work, were studied the Rashba effect in topological insulators without structural inversion symmetry. We performed a first principles study based on density functional theory to calculate the atomistic properties, formation energy and electronic structure. These results were used to development a effective Hamiltonian based on Zhang model.

They were studied two systems:

1) $\mathrm{Bi}_{2} \mathrm{Se}_{3}$ with $\mathrm{Sn}$ atoms deposited on the surface: This system can be seen as the Rashba effect manifestation on a topological insulator due to the structural inversion symmetry breaking. For a specific deposition site, the Sn atoms cause a reconstruction of the surface and display a third Dirac cone in the electronic structure. This third cone is not located on the surface and can be understood as the giant Rashba effect manifestation.

2) We propose a new non-centrosymmetric honeycomb-lattice QSH insulator family formed by the IV, V, and VII elements. The system formed by $\mathrm{Bi}, \mathrm{Pb}$ and $\mathrm{I}$ atoms is reported here as a new $2 \mathrm{D}$ topological insulator with Rashba effect. We show that this system has: i) Mechanical stability, ii) textit spin-splitting Rashba of $60 \mathrm{meV}$, iii) nontrivial energy gap of $0.14 \mathrm{eV}$, iv) backscattering forbidden for both edge and bulk conductivity channels in the nanoribbon band structure. These properties make PbBiI a good candidate to construct spintronic devices with less energy loss.

Keywords: Properties of solids, Ab-initio calculations, Density functional theory, Rashba effect, Topological insulator. 


\section{Conteúdo}

1 Introdução $\quad 2$

I Fundamentos 5

2 Teoria do funcional da densidade $\quad 6$

2.1 Equações de Kohn-Sham . . . . . . . . . . . . . . . . 9

2.2 Aproximações para o funcional de troca e correlação $E_{x c} \quad \ldots$. . . . 11

2.2.1 Aproximação da Densidade Local (LDA) . . . . . . . . . . . 11

2.2.2 Aproximação Generalizada do Gradiente (GGA) . . . . . . . . 12

2.3 Sistemas periódicos . . . . . . . . . . . . . . . . 13

2.3.1 O método de ondas planas . . . . . . . . . . . 15

2.3.2 O método de bases localizadas . . . . . . . . . . . 16

3 Efeito Rashba e Isolantes topológicos 18

3.1 Equação de Dirac . . . . . . . . . . . . . . . . . . . . . . 19

3.1.1 Limite de baixas energias . . . . . . . . . . . . 21 
3.1 .2 O acoplamento Spin-Órbita ............... 23

3.1.3 O acoplamento spin-órbita em átomos de hidrogenóides . . . . 24

3.1.4 O acoplamento spin-órbita em estruturas cristalinas . . . . . 27

3.1.5 O acoplamento spin-órbita Rashba . . . . . . . . . . 29

3.1.6 Efeito Hall de spin e Hall quântico de Spin . . . . . . . . . . . 31

$3.2 \mathrm{Bi}_{2} \mathrm{Se}_{3}$ e Família de Isolantes Topológicos (3D) . . . . . . . . . . 33

3.2.1 Propriedades estruturais . . . . . . . . . . . 35

3.2.2 Propriedades eletrônicas . . . . . . . . . . . . 38

3.2 .3 O modelo de Zhang . . . . . . . . . . . . . . 41

$\begin{array}{ll}\text { II Resultados } & 48\end{array}$

4 Sn adsorvido na superfície do $\mathrm{Bi}_{2} \mathrm{Se}_{3} \quad 49$

4.1 Propriedades estruturais do $S n / B i_{2} S e_{3} \ldots \ldots \ldots$. . . . . 50

4.2 Propriedades eletrônicas do $S n / B i_{2} S e_{3} \ldots \ldots$. . . . . . . . 53

4.3 Conclusão . . . . . . . . . . . . . . . . . 6 60

5 Um Novo Isolante Topológico - 2D $\quad 61$

5.1 Propriedades estruturais do PbBiI . . . . . . . . . . . 62

5.2 Propriedades eletrônicas do PbBiI . . . . . . . . . . . . . . . 64

5.3 Conclusão . . . . . . . . . . . . . . . . . . . . 72

6 Conclusões gerais $\quad 73$ 
$\begin{array}{ll}\text { A Aproximação de Born Oppenheimer } & 76\end{array}$

$\begin{array}{ll}\text { B Teoremas de Hohenberg-Kohn } & 78\end{array}$

$\begin{array}{ll}\text { C Variação do parâmetro da rede } & 81\end{array}$

$\begin{array}{ll}\text { D Estabilidade mecânica do PbBiI } & 83\end{array}$

E Artigo publicado

$\begin{array}{lr}\text { REFERENNCIAS } & 84\end{array}$ 


\section{Lista de Figuras}

3.1 Ilustração do splitting devido ao acoplamento spin-órbita (SOC) para um átomo hidrogenoide. Sem SOC (à esquerda; somente depende do número quântico principal $n$ ) e com SOC (à direita depende de $n$ e do número quântico de momento angular total $j$ ). . . . . . . . . 27

3.2 Ilustração do spin splitting devido ao efeito Rashba para um gás de elétrons livres. a) Sem SOC b) Esquerda: splitting para pequenas contribuições do efeito Rashba. Direita: splitting para grandes contribuições do efeito Rashba, também chamado "Giant Rashba spin

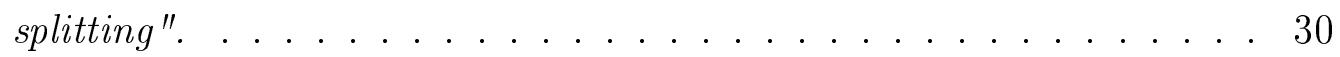

3.3 Ilustração do efeito Hall de spin [34]. . . . . . . . . . . . . . . 31

3.4 Ilustração da construção de um sistema com efeito Hall quântico de spin (isolante topológico) como uma superposição de dois subsistemas com efeito Hall de spin, um com topologia $N=1$ com spin up e outro com topologia $N=-1$ com spin down [34]. . . . . . . . . . 32

3.5 Ilustração dos estados de borda de isolantes 2D. a) Isolante trivial e b) Isolante topológico. As linhas sólidas e tracejadas denotam os estados de borda (um para cada borda) com spin opostos e velocidades opostas, devido à simetria de reversão temporal. [34]. . . . . . . . . . 34 
3.6 a) Supercélula hexagonal do $\mathrm{Bi}_{2} \mathrm{Se}_{3}$, com 15 camadas atômicas (3 QLs). Os vetores primitivos da célula unitária são representados por $t_{1}, t_{2}$ e $t_{3}$. b) Recorte no eixo $z$ da rede triangular, onde é observado três diferentes tipos de sítios não equivalentes. c) Primeira zona de Brillouin que contém 4 pontos TRIM (Time-Reversal-InvariantMomentum) e a superfície 2D (projeção da zona em 3D) da zona de Brillouin que está representada em linhas azuis. Imagem da referência $[23] \ldots \ldots \ldots \ldots \ldots \ldots \ldots$. . . . . . . . . . . . . . . . . .

3.7 Representação esquematica da inversão de banda dada no ponto $\Gamma$ para o bulk do sistema $B i_{2} S e_{3}$. A figura mostra a evolução dos orbitais atômicos $p_{x, y, z}$ do Bi e Se quando são considerados i) os efeitos das ligações químicas mais ii) a formação dos estados ligantes e antiligantes dada pela simetria de inversão mais iii) o campo cristalino e iv) interação de spin-órbita (SOC). Nota-se que existe uma inversão de bandas entre os estados $p_{z}$ do Bi e do Se quando o SOC é levada

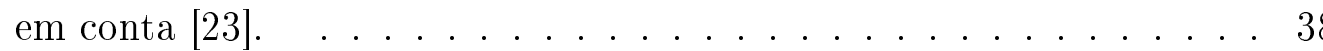

3.8 Estrutura de banda do bulk do sistema $\mathrm{Bi}_{2} \mathrm{Se}_{3}$ ao longo do caminho $\Gamma \rightarrow Z \rightarrow F \rightarrow \Gamma \rightarrow L$. a) Sem acoplamento spin-órbita (SOC). b) Com SOC. Em ambas são representadas as projeções dos orbitais $p_{z}$ do Bi (verde) e do Se (roxo) [44]. . . . . . . . . . . . 39

3.9 Estrutura de banda da superfície do sistema $B i_{2} S e_{3}$ para 1-6 quintuple layer (QL) ao longo do caminho $\bar{K} \rightarrow \bar{\Gamma} \rightarrow \bar{M}$; a) Sem acoplamento spin-órbita (SOC); b) Com SOC [44]. . . . . . . . . . . . . 40 
3.10 Representação ilustrativa dos estados de superfície de um isolante topológico (TI). a) Esquematização dos canais de condutividade com helicidade no spin formados no espaço real de um TI 3D. b) Dispersão de energia dos estados na superfície de um 3D TI que formam o cone de Dirac 2D. O spin é representado pelas setas. O spin é não degenerado, possuem helicidade e retroespalhamento proibido [45].

3.11 Dispersão de energia dado pelo modelo de Zhang [23] dos estados de bulk e superfície cerca do ponto $\Gamma$ nos filmes finos de $B i_{2} S e_{3}$ formados por $1-6$ QLs. . . . . . . . . . . . . . . . . . 47

4.1 Estrutura cristalina do $\mathrm{Sn} / \mathrm{Bi}_{2} \mathrm{Se}_{3}$, a) Vista lateral da QL superior discriminando as posições T1-3 b) Vista superior da geometria do

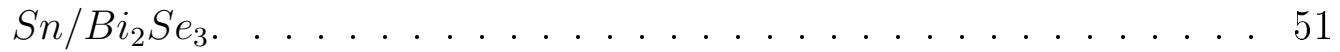

4.2 Barreira de potencial existente para passar da posição T3 a T3in no caso de um recobrimento de $100 \%$. A energia do sistema T3 é $\approx 0.06$ $\mathrm{eV}$ maior que o T3in. $\mathrm{h}=($ altura do $\mathrm{Sn})-($ Altura do Se1 $) . \quad \ldots . .53$

4.3 Estrutura eletrônica do sistemas $\mathrm{Sn} / \mathrm{Bi}_{2} \mathrm{Se}_{3}$ dado um recobrimento de 100\%, para os sítios a) T1, b) T2, c) T3 e d) T3in. . . . . . . 54

4.4 Sistema $S n / B i_{2} S e_{3}$ com deposição de Sn no sítio T3in com $100 \%$ de recobrimento; a) Estrutura cristalina após relaxação. O quadro vermelho (verde) encerra os átomos levados em conta na projeção dos orbitais mostrados em na dispersão de energia; b) Estrutura eletrônica com projeção nos orbitais dos átomos das superfícies. Os círculos com cores representam a contribuição dos orbitais dos átomos encerrados nas caixas na estrutura atômica com a cor respectiva. . . . . . . . 55 
4.5 Sistema $S n / B i_{2} S e_{3}$ com deposição de Sn no sítio T3in com $100 \%$ de recobrimento. Na estrutura eletrônica é projetada a contribuição dos orbitais $s\left(p_{z}\right)$ dos átomos de Se1 (Sn), é observado que o cone 1 é formado por uma hibridização dos orbitais $s$ dos Se1 e $p_{z}$ dos Sn. . . 56

4.6 Sistema $S n / B i_{2} S_{3}$ com deposição de Sn no sítio T3in com $100 \%$ de recobrimento. Textura de spin helicoidal para diferentes cortes nos cones mostrados na estrutura de bandas. As setas no plano representam as componentes $s_{x}, s_{y}$ do spin, enquanto que a escala de cor representa a componente $s_{z}$. É observado que a helicidade é oposta para cada par de cortes (superior e inferior) sobre o mesmo cone, e a helicidade é correspondente ao sistema protegido por simetria de reversão temporal. . . . . . . . . . . . . 57

4.7 Modelo efetivo. (a) Estrutura de bandas da superfície para 4 QLs considerando a quebra de simetria de inversão como um termo de Rashba. Os estados de superfície (bulk) são projetados em vermelho (azul). Nas bandas de condução do bulk (BCB1-3) é observado o splitting Rashba $E_{R}$. (b) Evolução do splitting Rashba em função do parâmetro de Rashba $\alpha_{R_{1}}$ para as bandas de condução do bulk

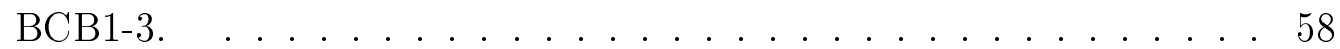

4.8 a) Efeito da mudança da massa efetiva na dispersão de energia da banda de condução do bulk BCB1. Os estados de superfície (bulk) são projetados em vermelho (azul); b) Relação da separação de energia $\Delta E$ dos cones da superfície com o shift de energia. c) Textura de spin para os cones 1, 2 e 3 do modelo efetivo considerando a quebra de simetria de inversão e modificação da massa efetiva. . . . . . . . . 59 
5.1 a) Vista lateral; b)Superior da estrutura atômica do PbBiI. O $h$ representa o comprimento da ligação do dímero IV-VII, enquanto que

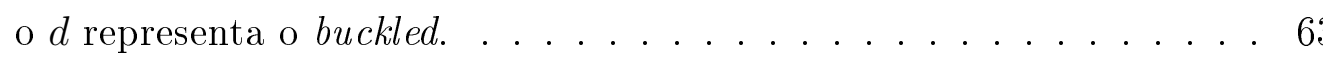

5.2 Estrutura de bandas a) Sem SOC e b) Com SOC. A escala de cor representa o peso da projeção sobre os orbitais $p_{z}$ e $p_{x}+p_{y}$ do $\mathrm{Bi}$, os quais são indicados por vermelho e azul respectivamente. . . . . . . . 64

5.3 Estrutura de bandas e textura de spin out-plane de (a) efeito Rashba usual e (b) o sistema PbBiI. As linhas pontilhadas na estrutura de banda representam os planos de energia (e1-e3 e e1'-e2') nas quais se observa a textura de spin in-plane, cujas grandezas são representadas pelo tamanho das setas. Na estrutura de bandas, as cores azul e vermelho representam a componente $\left\langle S_{z}\right\rangle$ do spin em unidades de $\hbar / 2$. As setas verdes mostram os processos de backscattering permitidos ou proibidos na estrutura de spin, no qual " $\mathrm{x}$ " indica um backscattering

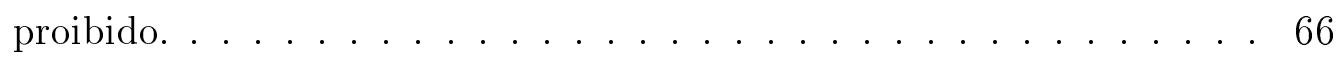

5.4 Figura esquemática da evolução da estrutura de bandas quando são incluídos os termos de: (I) Energia on-site e dispersão parabólica; (II) Efeito Rashba; (III) Termo de interação entre os estados com diferente momento angular total. A contribuição $\operatorname{dos} J=1 / 2(J=3 / 2)$ são representadas pelas linhas vermelhas(azuis). . . . . . . . . . 69

5.5 Estrutura de banda da nanofita armchair do PbBiI com $98.7 \AA$ de largura. A cor azul (vermelha) representa a projeção no spin up (down) para as componentes $x, y$ e $z$ do spin. . . . . . . . 71 
C.1 (a) Energia total do sistema em função da constante de rede para os funcionais de troca-correlação aproximação da densidade local (LDA) e aproximação generalizada do gradiente (GGA). (b) Gap de energia em função da constante de rede, onde $E_{g}$ e $E_{g \Gamma}$ são o gap do sistema e o gap no ponto $\Gamma$, respectivamente (ver figura 5.2 ). Abaixo da linha tracejada a 0.0, $E_{g \Gamma}$ é invertido. (c) spin-splitting Rashba em função da constante de rede. A seta representa a constante de rede para quando o sistema é energeticamente favorável segundo o funcional

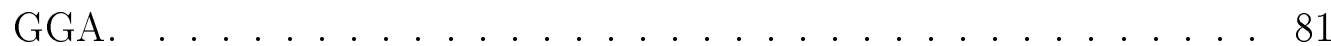

D.1 Dispersão de fônons para o sistema PbBiI calculado por primeiros princípios. 


\section{Lista de Tabelas}

3.1 Tabela dos níveis de energia para diferentes estados de um átomo hidrogenoide. $E_{N R}$ é a energia não relativística e $E_{S O C}$ a contribuição devido ao acoplamento spin-órbita (SOC). . . . . . . . . 26

3.2 Valores dos parâmetros, $a, c$ para diferentes elementos da família de isolantes topológicos $D_{3 d}^{5} \ldots \ldots \ldots \ldots \ldots \ldots$

3.3 Representações para as matrizes $\Gamma$ e os polinômios de $\boldsymbol{k}$ [46] . . . . . 44

4.1 Energia de adsorção por átomo depositado no respetivo sítio. . . . . . 51

5.1 Valores dos parâmetros do modelo tigh-binding efetivo para o sistema

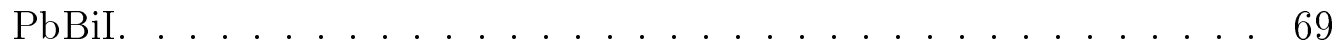




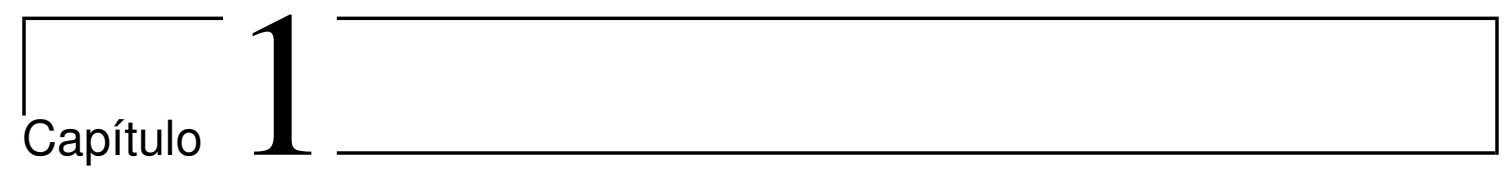

\section{Introdução}

O estudo das propriedades físicas de diversos sistemas pode ser feito através da combinação entre teoria, modelagem e simulação computacional. Estas três componentes formam uma ferramenta poderosa no entendimento e desenvolvimento de novos materiais. A Teoria do Funcional da Densidade (DFT), é formulada a partir dos princípios da mecânica quântica que é utilizada com sucesso na física da matéria condensada. Uma modelagem usa a teoria aplicada de forma analítica ou numérica para obter informação de um sistema específico. As simulações permitem controlar as condições em que o sistema se encontra, o que permite tratar fielmente o problema físico com um maior número de detalhes, de forma que as questões do problema surjam de forma natural.

$\mathrm{Na}$ última década, simulações computacionais contribuíram fortemente no avanço da nanotecnologia, dado ao esforço no estudo e desenvolvimento de novos materiais nanoestruturados. Particularmente, o entendimento dos fenômenos que envolvem o controle do spin constitui um ponto importante nesse campo e a spintrônica [1-3] nasceu com essa demanda. A chave desta está na detecção, manipulação e geração de correntes polarizadas de spin em semicondutores com baixa dimensionalidade [4,5]. Em nosso estudo em particular, o acoplamento spin- 
órbita é uma manifestação importante, o qual, causa um spin-splitting (quebra da degenerescência do spin) nas bandas de energia [6,7]. Em 2006 foi observado que poços quânticos de CdTe/HgTe possuem um acoplamento spin-órbita intrínseco o grande suficiente para apresentar Efeito Hall Quântico de Spin [8]. Materiais com esta propriedade foram chamados de Isolantes Topológicos e constituem um novo estado da matéria. O spin-splitting intrínseco em isolantes topológicos, permite o controle da polarização das correntes de spin via uma diferença de potencial [9]. O qual, permite utilizar estes sistemas como injetores de spin com logo tempo de relaxação do spin [10]. A quebra da degenerescência do spin devido à assimetria de inversão estrutural, pode ser descrita pela hamiltoniana efetiva de Rashba [11,12]

$$
H_{R}=\alpha\left(k_{y} \sigma_{x}-k_{x} \sigma_{y}\right)
$$

onde $\sigma_{x}$ e $\sigma_{y}$ são matrizes de Pauli e $\alpha$ o parâmetro de Rashba.

O objetivo deste trabalho é entender a anisotropia das estruturas de bandas de isolantes topológicos quando há um spin-splitting gerado pela assimetria de inversão estrutural.

Com esse objetivo usamos dois enfoques diferentes: i) cálculos de primeiros princípios baseados na Teoria do Funcional da Densidade e ii) modelos efetivos propostos por propriedades de simetria dependendo da estrutura cristalina do material.

O trabalho é dividido em duas partes principais, fundamentos e resultados. Na parte de fundamentos, no capítulo 2 são revisados os conceitos da Teoria do Funcional da Densidade e os métodos e aproximações utilizadas.

No capítulo 3 é apresentada a origem do acoplamento spin-órbita dentro da teoria de Dirac e algumas consequências desse acoplamento, tais como: o efeito Rashba, o efeitos Hall de spin e Hall quântico de spin, e a família de isolantes topológicos 
romboédricos 3D cujas propriedades topológicas são estudadas por cálculos de primeiros princípios e o modelo de Zhang.

Na parte de resultados, no capítulo 4 vamos apresentar os resultados do estudo de deposições de átomos de Sn sobre a superfície do isolante topológico $\mathrm{Bi}_{2} \mathrm{Se}_{3}$. As propriedades estruturais, energia de formação, e propriedades eletrônicas foram calculadas a partir de métodos de primeiros princípios baseados na Teoria do Funcional da Densidade. Também foi entendido este sistema com o modelo modificado de Zhang, no qual, é incluído o efeito Rashba para modelar a quebra de simetria de inversão estrutural.

No capítulo 5 propomos um novo isolante topológico 2D com efeito Rashba. Mostramos que a presença do efeito Rashba neste isolante topológico leva a um spinsplitting Rashba formado por bandas com textura de spin inusual. Esta textura de spin inusual leva ao retroespalhamento ser proibido tanto no estados de borda como nos estados de bulk no em torno do nível de Fermi.

Finalmente no capítulo 6 iremos apresentar as conclusões gerais das estruturas eletrônicas dadas pelo efeito Rashba em isolantes topológicos. 


\section{Parte I}

\section{Fundamentos}




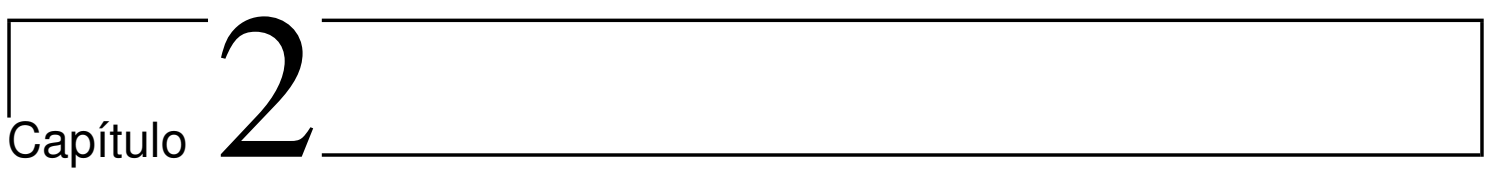

\section{Teoria do funcional da densidade}

Desde a formulação da Mecânica Quântica numa base mais sólida, elaborada por Bohr, Heisenberg, Schoedinger, Dirac, entre outros, a grande aspiração em física de materiais foi explicar suas propriedades elétricas, térmicas, óticas, magnéticas, etc, com base somente nos seus constituintes primários (átomos e estrutura atômica). A solução exata da equação de Schoedinger para sistemas de muitas partículas, onde a interação de Coulomb entre elétrons e núcleos está presente, é impraticável. Porém, é possível obter sua solução para o estado fundamental com grande precisão.

Este trabalho é um exemplo da aplicação desta teoria, conhecida como "Teoria do Funcional da Densidade (DFT)" que é o estado da arte no entendimento das propriedades dos materiais. Além do problema da interação elétron-elétron, não podemos ignorar o problema do acoplamento entre o movimento eletrônico e nuclear. A solução do auto estado global do sistema é impossível de achar e é necessário separar o movimento nuclear do eletrônico, conhecido como aproximação de Born-Oppenheimer [13] (Ver apêndice A).

No caso do tratamento de um sistema de muitos elétrons, pode-se mostrar, com um certo grau de aproximação, que é possível transformá-lo de um problema 
de N-corpos em N problemas de um corpo, como na teoria de Hartree-Fock. Nesse caso, a função de onda eletrônica total, que depende das coordenadas dos N elétrons, é o objeto fundamental. Será que existe uma outra maneira de resolver o problema? A resposta é sim, e o objeto fundamental passa a ser a densidade eletrônica total $\rho(r)$. A solução exata da equação de Schoedinger para o estado fundamental tendo a densidade como o principal objeto foi obtido por Hohenberg e Kohn em 1964 [14]. A teoria DFT tem sua raiz nos trabalhos de L.H. Thomas de 1927 [15], E. Fermi em 1928 [16] e P. Dirac em 1930 [17]. O trabalho de Thomas foi baseado em quatro suposições, de forma a tornar o problema de muitas partículas tratável: (i) correções relativísticas são desprezíveis; (ii) no átomo há um campo efetivo dado por um potencial $v$, dependendo somente da distância $r$ dos núcleos, tal que

$$
\begin{array}{crr}
v \longrightarrow 0 & \text { quando } & r \longrightarrow \infty \\
v r \longrightarrow Z e & \text { quando } & r \longrightarrow 0
\end{array}
$$

( onde $Z e$ é a carga nuclear ) (iii) os elétrons estão distribuídos uniformemente; e (iv) o potencial $v$ é por si mesmo determinado pela carga nuclear e sua distribuição eletrônica. O modelo, conhecido como Thomas-Fermi, leva-nos a uma energia total que é um funcional da densidade de carga $\rho$ da forma.

$$
E[\rho]=\left(\frac{3 h^{2}}{10 m}\right)\left(\frac{3}{8 \pi}\right)^{\frac{2}{3}} \int \rho^{\frac{5}{3}} d^{3} r-e \int \rho v_{N} d^{3} r-\frac{1}{2} e \int \rho v_{e} d^{3} r+U_{N N}
$$

onde $m$ é a massa do elétron, $v_{N}$ é o potencial devido aos núcleos e $v_{e}$ é o potencial devido aos elétrons. A primeira integral representa a energia cinética dos elétrons; A segunda, a energia devido a interação elétron-núcleo; A terceira, a expressão clássica da energia de interação elétron-elétron ou termo de Hartree; E o termo $U_{N N}$ a interação núcleo-núcleo.

Baseado nestes trabalhos, Hohenberg e Kohn mostraram que existe um funcional $E[\rho]$ exato que é relativamente mais simples de tratar. Esta teoria 
está alicerçada em 2 teoremas (ver demostrações no apêndice B), que podem ser enunciados da seguinte forma:

Teorema 1 O potencial externo $v(r)$ sentido pelos elétrons é um funcional único da densidade eletrônica $\rho(r)$.

Teorema 2 A densidade eletrônica exata de um sistema de muitos elétrons é a que minimiza o funcional de energia total $E[\rho]$.

Consequentemente, a energia pode ser escrita como um funcional da densidade eletrônica

$$
E[\rho(r)]=\int v(r) \rho(r) d r+F[\rho(r)]
$$

onde $F[\rho]$ é o funcional universal que tem a energia cinética e a energia de interação eletrônica

$$
F[\rho(r)] \equiv \frac{1}{2} \iint \frac{\rho(r) \rho\left(r^{\prime}\right)}{\left|r-r^{\prime}\right|} d^{3} r d^{3} r^{\prime}+T_{0}[\rho]+E_{x c}[\rho]
$$

o termo de energia cinética $T_{0}[\rho]$ corresponde à de um sistema não interagente com densidade $\rho$. Entretanto, as interações são embutidas no funcional de energia de intercâmbio e correlação $E_{x c}[\rho]$ e sendo, a fórmula exata deste, desconhecida analiticamente.

Nota-se que, na definição de $F[\rho(r)]$, não está presente o potencial externo $v(r)$. Portanto, o funcional (2.4) é válido para qualquer potencial externo e independe do número de partículas.

O seguinte passo no desenvolvimento da DFT foi feita por Kohn-Sham que formularam o problema usando um campo médio para achar os autoestados com certo grau de aproximação. 


\subsection{Equações de Kohn-Sham}

No desenvolvimento de Kohn-Sham [18] é mapeado o sistema de N elétrons interagentes em um sistema de $\mathrm{N}$ elétrons não interagentes com a mesma densidade eletrônica. Assume-se que as interações restantes são embutidas no termo de troca e correlação.

A densidade eletrônica que minimiza a energia do sistema pode ser obtida pelo método variacional, assim, ao considerar o funcional de energia

$$
E[\rho(r)]=T_{s}[\rho(r)]+\int v(r) \rho(r) d r+\frac{1}{2} \iint \frac{\rho(r) \rho\left(r^{\prime}\right)}{\left|r-r^{\prime}\right|} d r d r^{\prime}+E_{x c}[\rho(r)]
$$

e, ao levar em conta que o número de elétrons no sistema é fixo $\int \rho(r) d r=N$, a condição de mínimo para o funcional de energia $(\delta E[\rho(r)]=0)$ é restrita ao vínculo

$$
\frac{\delta}{\delta \rho(r)}\left(E[\rho(r)]-\mu\left[\int \rho(r) d r-N\right]\right)=0
$$

onde $\mu$ é um multiplicador de Lagrange, e tem sentido físico de potencial químico. Ao desenvolver esta equação obtêm-se

$$
\frac{\delta T_{s}[\rho(r)]}{\delta \rho(r)}+v^{K S}[\rho(r)]-\mu=0
$$

$\operatorname{com} v^{K S}[\rho(r)]$ o potencial efetivo de Kohn-Sham dado por

$$
v^{K S}[\rho(r)]=v(r)+\int \frac{\rho\left(r^{\prime}\right)}{r-r^{\prime}} d r^{\prime}+v_{x c}(\rho(r)),
$$

no qual $v_{x c}=\frac{\delta E_{x c}}{\delta \rho}$ é o potencial de troca-correlação. Ao considerar que o funcional de energia cinética é $T_{0}[\rho]=-\frac{1}{2} \int \psi_{i}^{*} \nabla^{2} \psi_{i} d r$ e a densidade de carga auxiliar é $\rho(r)=\sum_{i=1}^{N}\left|\psi_{i}(r)\right|^{2}$, então pode ser mapeado o sistema de muitos elétrons como um sistema de uma única partícula em um campo médio a partir do potencial efetivo 
(2.8). Esta é conhecida como equação de Kohn-Sham

$$
\left(-\frac{1}{2} \nabla^{2}+v^{K S}[\rho]\right) \psi_{i}(\boldsymbol{r})=\epsilon_{i} \psi_{i}(\boldsymbol{r})
$$

onde $\psi_{i}(\boldsymbol{r})$ são chamados orbitais de Kohn-Sham e são relacionados univocamente com a densidade eletrônica. Assim, uma vez conhecida a densidade eletrônica do estado fundamental $\rho_{o}(r)$ é possível calcular os orbitais $\psi_{0}(\boldsymbol{r})$ associada ao estado fundamental correspondente.

A implementação do ciclo autoconsistente pode ser da seguinte maneira:

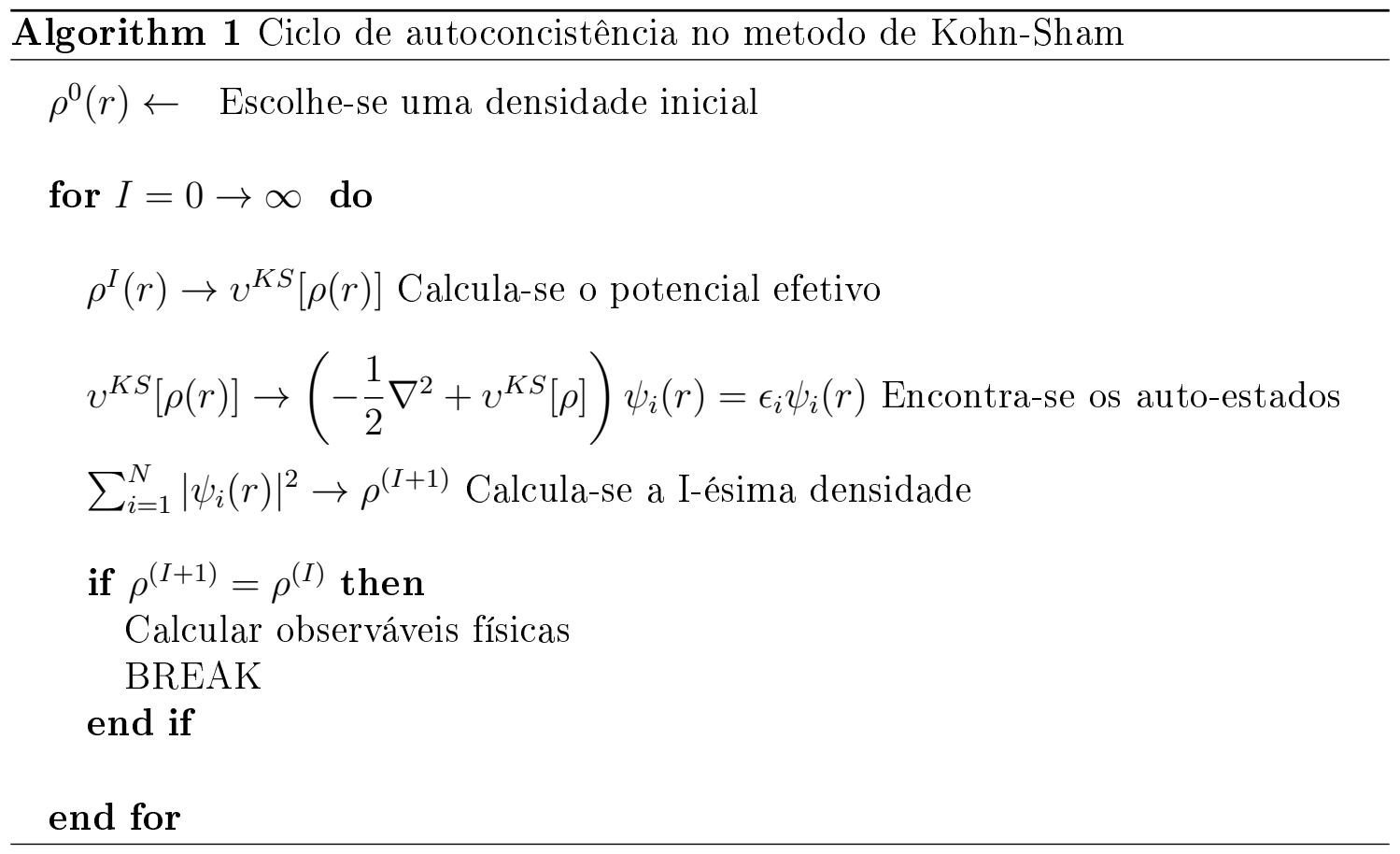

Para o cálculo do potencial de Kohn-Sham no siclo auto-consistente é preciso conhecer o funcional de troca e correlação $E_{x c}$. Na escolha deste termo foram desenvolvidas algumas aproximações. 


\subsection{Aproximações para o funcional de troca $\mathrm{e}$ correlação $E_{x c}$}

A forma analítica exata do funcional da energia de troca e correlação é desconhecida, porém, aproximações são feitas. As mais usadas são: i) Aproximação da Densidade Local (LDA) [18], onde supõe-se que o funcional de troca-correlação depende explicitamente apenas da densidade $\rho(r)$ e que esta densidade varia suavemente nas proximidades do ponto $r$; ii) Aproximação Generalizada do Gradiente (GGA) [19], na qual toma-se em conta a não homogeneidade da densidade que definirá o funcional de troca-correlação como dependente de $\rho(r)$ e do seu gradiente; iii) Funcionais Híbridos, que são combinações de duas o mais aproximações, entre outros.

\subsubsection{Aproximação da Densidade Local (LDA)}

O método de aproximação da densidade local (LDA) contido no trabalho original de Kohn-Sham [18]. Neste, a energia de troca-correlação para um gás de elétrons homogêneo de densidade $\rho(r)$ no ponto $r$ é assumida igual à energia de trocacorrelação de um gás de elétrons homogêneos com a mesma densidade. Supõe-se que $\rho(r)$ varia suavemente nas proximidades do ponto $r$, assim, escreve-se

$$
E_{x c}^{L D A}[\rho]=\int \rho(r) \epsilon_{x c}^{h}(\rho(r)) d \boldsymbol{r}
$$

sendo $\epsilon_{x c}^{h}(\rho(r))$ a energia de troca-correlação por elétron de um gás de elétrons homogêneo com densidade $\rho(r)=\rho$. $\epsilon_{x c}^{h}$ é dividido em um termo de correlação $\epsilon_{c}$ mais o termo de troca $\epsilon_{x}$. Desta forma, o problema fica dividido em dois, sendo o 
termo de troca dado por

$$
\epsilon_{x}(\rho)=-\frac{e^{2}}{4 \pi \varepsilon_{0}} \frac{3}{4}\left(\frac{3}{\pi}\right)^{1 / 3} \rho^{1 / 3}=-\frac{e^{2}}{4 \pi \varepsilon_{0} a_{0}} \frac{0,4582}{r_{s}}
$$

com $r_{s}$ o raio de Wigner-Seitz $\left(r_{s} a_{0}=\left(\frac{3}{4 \pi} \rho^{-1}\right)^{1 / 3}\right.$ em unidades atômicas) e $a_{0}$ é o raio de Bohr.

Por outro lado, a energia de correlação não pode ser determinada exatamente. Celperley e Alder [20] calcularam numericamente $\epsilon_{c}$ para vários valores da densidade, e foi obtido pelo método de Monte Carlo Quântico para um gás de elétrons homogêneo e interagentes. Vários autores têm parametrizado $\epsilon_{c}$ em função de $r_{s}$, por exemplo, Perdew e Zunger [21] reportaram a seguinte parametrização:

$$
\begin{gathered}
\epsilon_{c}=\frac{0,1423}{1+1,9529 \sqrt{\frac{r_{s}}{a_{0}}}+0,3334 \frac{r_{s}}{a_{0}}} ; r_{s} \geq 1 \\
\epsilon_{c}=-0,0480+0,0311 \ln \frac{r_{s}}{a_{0}}-0,0116 \frac{r_{s}}{a_{0}}+0,0020 r_{s} \ln \frac{r_{s}}{a_{0}} ; r_{s}<1 .
\end{gathered}
$$

Para os casos no qual a densidade eletrônica é fortemente não uniforme, os resultados com LDA diferem consideravelmente dos experimentais. Para estes sistemas é necessário uma aproximação que leve em conta a variação da densidade eletrônica.

\subsubsection{Aproximação Generalizada do Gradiente (GGA)}

Para melhorar a LDA são incluídas as correções dadas pela variação da densidade, deixando o funcional de energia $E_{x c}$ em função da densidade e do gradiente. Isto é conhecido como Aproximação do Gradiente Generalizado (Generalized Gradient Approximation, GGA) [19]:

$$
E_{x c}^{G G A}[\rho]=\int \rho(r) \epsilon_{x c}^{h}(\rho(r)) F_{x c}(\nabla \rho(r)) d r
$$


onde $F_{x c}$ é uma correção funcional determinada. Entre os $F_{x c}$ mais usados esta o proposto em 1996 por Perdew - Burke - Erzenhot [22] conhecido como PBE

$$
F_{x c}^{P B E}(s)=1,804-\frac{0,646}{0,804+0,220 s^{2}}
$$

com $s=\frac{|\nabla \rho|}{\sqrt[3]{3 \pi^{2} \rho^{4}}}$ o gradiente adimensional da densidade. Existem outros tipos de funcionais propostos na literatura que são amplamente usados: LYPB (Lee - Yang - Parr - Becke) [58, 59]; PW (Perdew - Wang) [19]; entre outros.

O desenvolvimento de funcionais melhorados atualmente é uma área de pesquisa muito ativa. Embora a GGA leve a resultados mais acertados nas descrições do gap de energia e de ligações químicas, também possui algumas limitações: superestima o parâmetro de rede, não inclui as interações de tipo Van der Waals. Neste trabalho utilizamos LDA no código SIESTA e GGA no código VASP, para obter os resultados ab-initio apresentados nos capítulos 4 e 5 .

\subsection{Sistemas periódicos}

Os sólidos cristalinos são formados segundo um arranjo periódico pela repetição de uma unidade estrutural. Em física do estado sólido, diz-se que os cristais são formados pela união de uma rede de Bravais e uma base. Esta base é constituída por um ou vários átomos (ou moléculas). Toda rede de Bravais possui simetria para translações espaciais, então, é possível definir um conjunto de vetores da rede $\left\{\boldsymbol{R}_{\boldsymbol{n}}\right\}$, de maneira que, quando uma operação de simetria $t \boldsymbol{R}_{\boldsymbol{n}}$ é aplicado ao sistema, o sistema resultante será completamente equivalente ao inicial. O potencial gerado pelos átomos da base também obedece esta simetria

$$
V\left(\boldsymbol{r}+\boldsymbol{R}_{\boldsymbol{n}}\right)=V(\boldsymbol{r})
$$


o que significa que o potencial é periódico. Consequentemente, a densidade eletrônica do estado fundamental e o potencial efetivo na equação de Kohn-Sham também são periódicos.

$$
\left(-\frac{1}{2} \nabla^{2}+v^{K S}[\rho(\boldsymbol{r})]\right) \psi_{i}(\boldsymbol{r})=\epsilon_{i} \psi_{i}(\boldsymbol{r})
$$

$\operatorname{com} \rho(\boldsymbol{r})=\rho\left(\boldsymbol{r}+\boldsymbol{R}_{\boldsymbol{n}}\right)$ e $v^{K S}[\rho(\boldsymbol{r})]=v^{K S}\left[\rho\left(\boldsymbol{r}+\boldsymbol{R}_{\boldsymbol{n}}\right)\right]$.

Os elétrons que obedecem a equação (2.17) com um potencial periódico, são conhecidos como elétrons de Bloch e obedecem ao teorema de Bloch.

Teorema de Bloch: As autofunções $\psi$ de um hamiltoniano monoeletrônico $H=$ $-\frac{\hbar^{2} \nabla^{2}}{2 m}+V(\boldsymbol{r})$, onde $V\left(\boldsymbol{r}+\boldsymbol{R}_{\boldsymbol{n}}\right)=V(\boldsymbol{r})$ para todos os $\boldsymbol{R}_{\boldsymbol{n}}$ em uma rede de bravais, pode ser escolhido como um produto de uma onda plana e uma função com a periodicidade da rede,

$$
\psi_{n k}(\boldsymbol{r})=e^{i \boldsymbol{r} \cdot \boldsymbol{k}} u_{n k}(\boldsymbol{r}),
$$

onde $u_{n k}(\boldsymbol{r})=u_{n k}\left(\boldsymbol{r}+\boldsymbol{R}_{\boldsymbol{n}}\right)$ é conhecida como função de Bloch.

O teorema de Bloch implica que os estados próprios de $H$ devem ser tomados de forma tal que para cada $\psi$ está associado a um vetor de onda $\boldsymbol{k}$ que satisfaz

$$
\psi\left(\boldsymbol{k}, \boldsymbol{r}+\boldsymbol{R}_{\boldsymbol{n}}\right)=e^{i \boldsymbol{k} \cdot \boldsymbol{R}_{\boldsymbol{n}}} \psi(\boldsymbol{k}, \boldsymbol{r})
$$

para todo $\boldsymbol{R}_{\boldsymbol{n}}$ da rede de Bravais. Este teorema é interpretado como sendo uma condição de contorno das soluções da equação de Schrödinger para um potencial periódico. Os autovalores $E_{\boldsymbol{k}}$ e as autofunções $\psi(\boldsymbol{k}, \boldsymbol{r})$ dos estados monoeletrônicos são, então, classificados através dos vetores de onda $\boldsymbol{k}$. 


\subsubsection{O método de ondas planas}

Ao considerar $\vec{G}_{n}$ o vetor de traslação da rede recíproca, que obedece a relação $e^{v e c G_{n} \cdot v e c R_{n}}$. Um estado eletrônico $k$-ésimo pode-se expandir em uma série de Fourier na forma

$$
\Psi_{k}(r)=\sum_{\vec{G}_{n}} C_{k+\vec{G}_{n}} e^{i\left(k+\vec{G}_{n}\right) \cdot r},
$$

expande-se também o potencial periódico de Kohn-Sham

$$
V(r)=\sum_{\vec{G}_{n}} V^{G} e^{i \vec{G}_{n} \cdot r}
$$

Ao substituir as equações $(2.20)$ e (2.21) na (2.17), multiplicá-las por $e^{-i\left(k+\vec{G}_{n}\right) \cdot r}$ à esquerda e integrando no espaço de uma célula unitária $v_{u}$, tem-se:

$$
\left[-\frac{\hbar^{2}}{2 m}\left(k+\vec{G}_{n}\right)^{2}\right] C_{k+\vec{G}_{n}}-\sum_{\vec{G}_{n}} V_{\vec{G}_{n}-\vec{G}_{n}^{\prime}} C_{k+\vec{G}_{n}^{\prime}}=\epsilon C_{k+\vec{G}_{n}}
$$

onde

$$
V_{\vec{G}_{n}-\vec{G}_{n}^{\prime}}=\int_{v_{u}} V(r) e^{i\left(\vec{G}_{n}-\vec{G}_{n}^{\prime}\right) \cdot r} d r
$$

Os autovalores de energia que aparecem em (2.22) são obtidos ao resolver a equação secular

$$
\operatorname{det}\left|\left(-\frac{\hbar^{2}}{2 m}\left(k+\vec{G}_{n}\right)^{2}+\epsilon\right) \delta_{\vec{G}_{n} \vec{G}_{n}^{\prime}}-V_{\vec{G}_{n}-\vec{G}_{n}^{\prime}}\right|=0
$$

A quantidade de ondas utilizadas é determinada em função de uma energia de corte $E_{c u t}$. Assim, toda onda planas com uma energia cinética menor que $E_{c u t}$ são incluídas na base.

O método de ondas planas é robusto, dado que estas possuem a informação da periodicidade do sistema. Entretanto, a descrição apropriada de sistemas maiores exige muitas ondas planas o que pode levar a problemas de convergência. 


\subsubsection{O método de bases localizadas}

O método de bases localizadas utiliza um conjunto de funções de onda associada aos sítios atômicos. Assume-se que na vizinhança de cada ponto da rede direta, o hamiltoniano total pode ser aproximado por um hamiltoniano de sítio local periódico. Se o sistema tem N sítios, onde cada um deles possui uma função de onda associada, a autofunção do hamiltoniano total será uma combinação linear destas N autofunções de um sítio

$$
\psi_{n \vec{k}}(\vec{r})=\sum_{\vec{R}_{n}} \omega_{n \vec{k}}\left(\vec{r}-\vec{R}_{n}\right) e^{i \vec{R}_{n} \cdot \vec{k}}
$$

sendo $\omega_{n \vec{k}}\left(\vec{r}-\vec{R}_{n}\right)$ funções de Wannier centradas em cada base.

Expande-se $\omega_{n \vec{k}}$ usando um conjunto de bases localizadas $\phi_{\mu}\left(\vec{r}-\overrightarrow{P_{\mu}}\right)$ centradas em átomos situados em $\vec{P}_{\mu}$ (aqui $\vec{P}_{\mu}$ leva em conta os pontos da rede de Bravais que contem mais de que um átomo)

$$
\omega_{n \vec{k}}\left(\vec{r}-\vec{R}_{n}\right)=\sum_{\mu} C_{i \vec{k}}^{\mu} \phi_{\mu}\left(\vec{r}-\vec{P}_{\mu}-\vec{R}_{n}\right)
$$

ao substituir na hamiltoniana de Kohn-Sham (2.17), obtém-se

$$
\begin{array}{r}
\sum_{\mu}\left[\sum_{R_{n}} e^{i \vec{R}_{n} \cdot \vec{k}}\left(-\frac{1}{2} \nabla^{2}+v^{K S}(r)\right) \phi_{\mu}\left(\vec{r}-\vec{P}_{\mu}-\vec{R}_{n}\right)\right] C_{i \vec{k}}^{\mu} \\
=\epsilon_{i} \sum_{\mu} \sum_{R_{n}}\left[e^{i \vec{R}_{n} \cdot \vec{k}} \phi_{\mu}\left(\vec{r}-\vec{P}_{\mu}-\vec{R}_{n}\right)\right]
\end{array}
$$

Ao multiplicar a expressão anterior por uma função base $\phi_{\nu}^{*}\left(\vec{r}-\vec{P}_{\nu}\right)$ e integrá-la no espaço real, resulta:

$$
\sum_{\mu} H_{\nu \mu}(\vec{k}) C_{i \vec{k}}^{\mu}=\epsilon_{n} \sum_{\mu} S_{\nu \mu}(\vec{k}) C_{i \vec{k}}^{\mu}
$$


com

$$
H_{\nu \mu}(\vec{k})=\sum_{\vec{R}_{n}} e^{i \vec{R}_{n} \cdot \vec{k}}\left[\int d \vec{r} \phi_{\nu}^{*}\left(\vec{r}-\vec{P}_{\nu}\right)\left(-\frac{1}{2} \nabla^{2}+v^{K S}(r)\right) \phi_{\mu}\left(\vec{r}-\vec{P}_{\mu}-\vec{R}_{n}\right)\right]
$$

e

$$
S_{\nu \mu}(\vec{k})=\sum_{\vec{R}_{n}} e^{i \vec{R}_{n} \cdot \vec{k}}\left[\int d \vec{r} \phi_{\nu}^{*}\left(\vec{r}-\vec{P}_{\nu}\right) \phi_{\mu}\left(\vec{r}-\vec{P}_{\mu}-\vec{R}_{n}\right)\right]
$$

Normalmente, as funções base $\phi_{\nu}\left(\vec{r}-\vec{P}_{\nu}\right)$ têm formato de orbitais atômicos de tal modo que estas funções constituem uma base de orbitais atômicos localizados (LCAO).

Neste trabalho é usado o código computacional VASP, o qual, calcula uma uma solução aproximada da equação de Schrödinger de muitos corpos, dentro da Teoria do Funcional da Densidade, por meio da solução da equação de Kohn-Sham. No VASP, as interações centrais, tais como os orbitais eletrônicos, a densidade de carga eletrônica, e o potencial local são expandidos em uma base de ondas planas. Também é utilizado o código SIESTA o qual permite realizar cálculo de estruturas eletrônicas de forma eficiente dada à utilização do método de bases localizadas. 


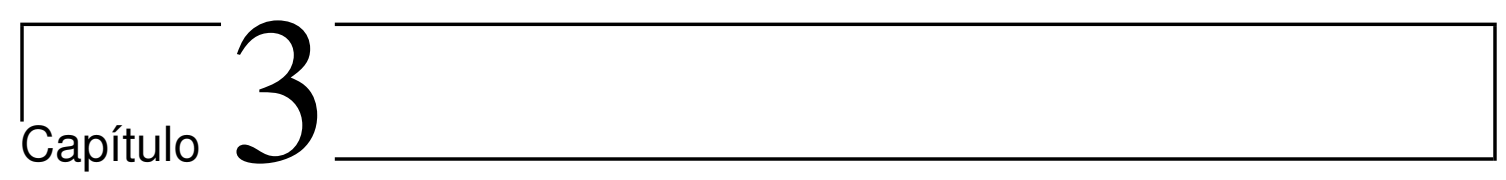

\section{Efeito Rashba e Isolantes topológicos}

É conhecido que a solução da equação de Schrödinger, em cálculos de sistemas formados por átomos leves, fornece resultados que, de um modo geral, concordam com a experiência. Porém, apresenta algumas discrepâncias qualitativas e quantitativas para sistemas com átomos pesados. Isto se dá, em grande parte, à equação de Schrödinger não incluir os efeitos associados as correções relativísticas e o funcional de energia não descrever corretamente os efeitos de correlação eletrônica.

Neste capítulo, mostraremos que a equação de Dirac fornece três termos que podem ser incorporados à equação de Schrödinger que chamamos de correções relativísticas i) Correção de energia cinética; ii) Termo de Darwin e iii) acoplamento spin-órbita (SOC; spin-orbit coupling). Também estudaremos alguns fenômenos que são consequência das correções relativísticas, tais como: O efeito Rashba, o efeito Hall de spin, o efeito Hall quântico de spin, e algumas propriedades dos isolantes topológicos.

O SOC é um efeito associado à interação do spin com o momento angular orbital do elétron. Este, causa vários efeitos nas propriedades eletrônicas, como a quebra de degenerescência, spin-spliting e shifting nas bandas de energia. Quanto mais pesados são os átomos considerados, mais pronunciados tais efeitos. O SOC 
é importante no estudo de alguns isolantes topológicos, levando em conta vários aspectos: A família de isolantes topológicos 3D romboédricos são formados por átomos pesados [23]; O SOC preserva a simetria de reversão temporal e dá origem à transição de fase topológica via inversão de bandas.

\subsection{Equação de Dirac}

O conceito de spin é introduzido inicialmente de forma $a d$ hoc na equação de Schrödinger, dado que, é necessária a existência do momento magnético intrínseco para explicar as deflexões observadas no experimento de Stern e Gerlach. Desde o ponto de vista teórico, Dirac em 1928 [24] mostrou que o spin do elétron é, de fato, naturalmente derivado da combinação entre mecânica quântica e relatividade especial.

Da relatividade especial, a energia de uma partícula livre relativística é definida por

$$
\epsilon^{2}=(c p)^{2}+\left(m c^{2}\right)^{2}
$$

A proposta de Dirac consiste na linearização da expressão (3.1), dada pela introdução de dois coeficientes $\boldsymbol{\alpha}$ e $\beta$. Assim, o problema é proposto como:

$$
\left(\epsilon+c \boldsymbol{\alpha} \cdot \mathbf{p}+\beta m c^{2}\right)\left(\epsilon-c \boldsymbol{\alpha} \cdot \mathbf{p}-\beta m c^{2}\right)=0
$$

A relação (3.2) implica que:

$$
c \boldsymbol{\alpha} \cdot \mathbf{p}+\beta m c^{2}=\epsilon .
$$

Ao elevar ao quadrado (3.3) e substituir em (3.1) mostra-se que a relação (3.1) será 
satisfeita sempre que $\boldsymbol{\alpha}$ e $\beta$ cumpram

$$
\begin{array}{cc}
\alpha_{i}^{2}=\beta^{2}=1 & (i=x, y, z) \\
\alpha_{i} \alpha_{j}+\alpha_{j} \alpha_{i}=0 & (i \neq j) \\
\alpha_{0} \beta+\beta \alpha_{i}=0 . &
\end{array}
$$

Os coeficientes $\boldsymbol{\alpha}$ e $\beta$ anticomutam, então, eles não podem ser valores escalares. Dirac propôs uma possível combinação, onde $\boldsymbol{\alpha}$ e $\beta$ são matrizes $4 \mathrm{x} 4$, conhecida como representação de Dirac-Pauli:

$$
\alpha_{i}=\left(\begin{array}{cc}
0 & \sigma_{i} \\
\sigma_{i} & 0
\end{array}\right), \quad \beta=\left(\begin{array}{cc}
I_{2 x 2} & 0 \\
0 & -I_{2 x 2}
\end{array}\right)
$$

aqui $\sigma_{i}$ são as matrizes de Pauli definidas como

$$
\sigma_{x}=\left(\begin{array}{cc}
0 & 1 \\
1 & 0
\end{array}\right), \quad \sigma_{y}=\left(\begin{array}{cc}
0 & -i \\
i & 0
\end{array}\right), \quad \sigma_{z}=\left(\begin{array}{cc}
1 & 0 \\
0 & -1
\end{array}\right)
$$

A representação usada é escrita em blocos 2x2, o estado quântico é descrito como

$$
\mid \Psi>=\left(\begin{array}{c}
\mid \phi> \\
\mid \psi>
\end{array}\right)
$$

com

$$
\left|\phi>=\left(\begin{array}{c}
\mid \chi_{1}> \\
\mid \chi_{2}>
\end{array}\right),\right| \psi>=\left(\begin{array}{c}
\mid \chi_{3}> \\
\mid \chi_{4}>
\end{array}\right)
$$

Ou seja, que a função de onda (3.7) é um vetor de 4 componentes. Ao representar (3.3) como uma equação de valores próprios, chega-se à expansão relativística da equação de Schrödinger

$$
H \Psi=\left(\boldsymbol{\alpha} \cdot \boldsymbol{p} c+\beta m c^{2}\right) \Psi,
$$


a qual, é conhecida como equação de Dirac.

Entretanto, o momento angular orbital $\boldsymbol{L}$ não comuta com o hamiltoniano (3.9). Assim, $\boldsymbol{L}$ não se conserva, enquanto que, o momento angular total $\boldsymbol{J}=\boldsymbol{L}+\boldsymbol{S}$ $\operatorname{sim}$ (sendo $\boldsymbol{S}$ o operador de momento angular do spin).

Na base de $\mid \phi>$ e $\mid \psi>$ a equação de Dirac para a partícula livre torna-se um conjunto de equações acopladas

$$
\begin{gathered}
\left(H-m c^{2}\right)|\phi>=(\boldsymbol{\sigma} \cdot \boldsymbol{p})| \psi> \\
\left(H+m c^{2}\right)|\psi>=(\boldsymbol{\sigma} \cdot \boldsymbol{p})| \phi>.
\end{gathered}
$$

Quando a partícula estiver dentro de um potencial externo $V$, as equações de Dirac se tornam

$$
\begin{aligned}
& \left(H-V-m c^{2}\right)|\phi>=(\boldsymbol{\sigma} \cdot \boldsymbol{p})| \psi> \\
& \left(H-V+m c^{2}\right)|\psi>=(\boldsymbol{\sigma} \cdot \boldsymbol{p})| \phi>
\end{aligned}
$$

\subsubsection{Limite de baixas energias}

No limite de baixas energias, a energia total de uma partícula relativística equivale à energia não relativística mais os termos de correção. De forma equivalente espera-se esse resultado na mecânica quântica.

Da equação (3.13) tem-se

$$
\left|\psi>=\left(\frac{E-V}{2 m c^{2}}+1\right)^{-1} \frac{(\boldsymbol{\sigma} \cdot \boldsymbol{p})}{2 m c^{2}}\right| \phi>
$$

Ao desacoplar as equações (3.13) e (3.12) obtêm-se uma forma compacta, 
que para $\mid \phi>$ resulta

$$
E_{t}\left|\phi>=(\boldsymbol{\sigma} \cdot \boldsymbol{p})\left(\frac{E-V}{2 m c^{2}}+1\right)^{-1} \frac{(\boldsymbol{\sigma} \cdot \boldsymbol{p})}{2 m c^{2}}\right| \phi>.
$$

No limite de baixas energias $E-V \ll 2 m c^{2}$, então, pode-se escrever a aproximação

$$
\left(\frac{E-V}{2 m c^{2}}+1\right)^{-1} \approx 1-\frac{E-V}{2 m c^{2}} .
$$

No caso de $V$ ser um potencial central $V(r)$, cumpre-se que:

$$
\boldsymbol{p} V(\boldsymbol{r})-V(\boldsymbol{r}) \boldsymbol{p}=-i \hbar \nabla V(\boldsymbol{r})
$$

de modo que (3.15) fica

$$
E_{t}\left|\phi>=\frac{\boldsymbol{p}^{2}}{2 m}\right| \phi>+V(\boldsymbol{r})\left|\phi>-(\boldsymbol{\sigma} \cdot \boldsymbol{p})\left(\frac{E-V(\boldsymbol{r})}{4 m^{2} c^{2}}\right)(\boldsymbol{\sigma} \cdot \boldsymbol{p})\right| \phi>
$$

Nota-se que, em ordem zero, recupera-se a equação de Schödinger não relativística. Consequentemente, o Hamiltoniano pode ser escrito como $H_{t}=H_{0}+H_{1}$, sendo $H_{0}$ o termo não relativístico e $H_{1}$ o termo de aproximação de primeira ordem.

E o termo de correção pode-se escrever como:

$$
\begin{aligned}
(\boldsymbol{\sigma} \cdot \boldsymbol{p}) V(\boldsymbol{r})(\boldsymbol{\sigma} \cdot \boldsymbol{p}) & =-\hbar^{2} V(\boldsymbol{r}) \nabla^{2}-\hbar^{2} \nabla V(\boldsymbol{r}) \cdot \nabla V(\boldsymbol{r})-\frac{\hbar^{2}}{r} \frac{d V(\boldsymbol{r})}{d r} i \boldsymbol{\sigma} \cdot(\boldsymbol{r} \times \nabla) \\
& =\frac{1}{2}\left(\boldsymbol{p}^{2} V(\boldsymbol{r})+V(\boldsymbol{r}) \boldsymbol{p}^{2}\right)+\frac{\hbar^{2}}{2} \nabla^{2} V(\boldsymbol{r})+\frac{2}{r} \frac{d V(\boldsymbol{r})}{d r} \boldsymbol{L} \cdot \boldsymbol{S}
\end{aligned}
$$

Então,

$$
H_{1}=-\frac{\boldsymbol{p}^{4}}{8 m^{3} c^{2}}+\frac{\hbar^{2}}{4 m^{2} c^{2}} \nabla^{2} V(\boldsymbol{r})-\frac{\hbar}{4 m^{2} c^{2}} \boldsymbol{\sigma} \cdot(\boldsymbol{p} \times \nabla V) .
$$

Deste resultado, observa-se os três termos de correção relativísticas, usualmente discriminados como: 
Correção de energia cinética.

$$
T_{\text {rel }}=-\frac{\boldsymbol{p}^{4}}{8 m^{3} c^{2}}
$$

\section{Termo de Darwin}

$$
H_{D}=\frac{\hbar^{2}}{4 m^{2} c^{2}} \nabla^{2} V(\boldsymbol{r})
$$

\section{Acoplamento spin-órbita}

$$
H_{S O C}=-\frac{\hbar}{4 m^{2} c^{2}} \boldsymbol{\sigma} \cdot(\boldsymbol{p} \times \nabla V)
$$

O acoplamento spin-órbita (SOC; "Spin-Orbit coupling", também chamado efeito spin-órbita ou interação de spin-órbita) é indispensável na determinação do espectro atômico quando se trata de átomos com momento angular intrínseco grandes (átomos pesados). O efeito imediato do SOC sobre a estrutura eletrônica é a quebra da degenerescência dos níveis de energia. Isto causa splitting nas bandas de energia para todo ponto que não seja um TRIM(Time reversal invariant momentum); Isto é, todo ponto que não for um invariante com transformações de simetria de reversão temporal.

\subsubsection{O acoplamento Spin-Órbita}

O acoplamento spin-órbita também pode ser ilustrado no campo da eletrodinâmica clássica, na qual os vetores dos campos eletromagnéticos dependem do sistema de referência. Assim, um elétron que está em movimento, com velocidade $\boldsymbol{v}$ perpendicular a um campo elétrico $\boldsymbol{E}$, pelos efeitos relativísticos, experimentará um campo magnético $\boldsymbol{B}$ de magnitude

$$
\boldsymbol{B}=-\frac{1}{c} \boldsymbol{v} \times \boldsymbol{E}=\frac{1}{m c}(\boldsymbol{E} \times \boldsymbol{p}),
$$


no qual os termos de ordem $\left(\frac{v}{c}\right)^{2}$ ou maior são desprezados. Assim, a energia que ganha o elétron neste campo, devido ao momento magnético $\boldsymbol{\mu}$ seria

$$
\begin{aligned}
-\boldsymbol{\mu} \cdot \boldsymbol{B} & =\quad-\frac{1}{m c} \boldsymbol{S} \cdot \boldsymbol{B} \\
& =-\frac{1}{m^{2} c^{2}} \boldsymbol{S} \cdot(\boldsymbol{E} \times \boldsymbol{p}) .
\end{aligned}
$$

Expressando $\boldsymbol{S}=\frac{\hbar}{2} \boldsymbol{\sigma}$, e $\boldsymbol{E}=-\nabla V$,

$$
-\boldsymbol{\mu} \cdot \boldsymbol{B}=-\frac{\hbar}{2 m^{2} c^{2}} \boldsymbol{\sigma} \cdot(\boldsymbol{p} \times \nabla V) .
$$

Este termo de energia adicional é essencialmente o termo de acoplamento spinórbita (3.23), exceto por um fator de 2. A explicação mais satisfatória dada a esta discrepância foi feita por Llewellyn Thomas [25] e é conhecida como precessão de Thomas.

\subsubsection{O acoplamento spin-órbita em átomos de hidrogenóides}

Ao considerar o movimento orbital do elétron em um potencial central $V(\boldsymbol{r})$, que depende apenas da distância ao núcleo $\boldsymbol{r}$, o acoplamento spin-órbita torna-se:

$$
\begin{aligned}
H_{S O C} & =-\frac{\hbar}{4 m^{2} c^{2}} \boldsymbol{\sigma} \cdot(\boldsymbol{p} \times \nabla V(\boldsymbol{r})) \\
& =-\frac{\hbar}{4 m^{2} c^{2}} \frac{1}{r} \frac{\partial V}{\partial r} \boldsymbol{\sigma} \cdot(\boldsymbol{p} \times \boldsymbol{r}) \\
& =\frac{1}{2 m^{2} c^{2}} \frac{1}{r} \frac{\partial V}{\partial r} \boldsymbol{L} \cdot \boldsymbol{S} .
\end{aligned}
$$

Espera-se que a maior contribuição ao SOC resulte da região imediata ao núcleo, dado que a derivada radial do potencial é maior nas suas vizinhanças. Para $\boldsymbol{r}$ pequenos, o potencial nuclear pode ser tratado como sendo Coulombiano $V(r)=$ 
$-\frac{Z}{r}$. Sua derivada seria proporcional ao número atômico $Z$. Adicionalmente, $E_{S O C}=<\psi\left|H_{S O C}\right| \psi>$, então, $E_{S O C}$ será dependente quadraticamente de $|\psi\rangle$ e toda vez que a função de onda depende de $Z$, os efeitos do SOC aumentam. Isto também é válido para um cristal.

O Hamiltoniano relativístico do átomo hidrogenoide não comuta com os operadores $\boldsymbol{L}$ e $\boldsymbol{S}$. Estes não são contantes de movimento e seus valores esperados, $j$ e $s$ respetivamente, não são bons números quânticos. Enquanto que, o momento angular total $\boldsymbol{J}=\boldsymbol{L}+\boldsymbol{S}$ comuta e é uma constante de movimento. Consequentemente, $\boldsymbol{J}^{2}$ também comuta com este. Entretanto, o valor esperado de $\boldsymbol{S} \cdot \boldsymbol{L}$ é

$$
\boldsymbol{S} \cdot \boldsymbol{L}\left|\Psi>=\frac{1}{2}\left(\boldsymbol{J}^{2}-\boldsymbol{L}^{2}-\boldsymbol{S}^{2}\right)\right| \Psi>=\frac{\hbar^{2}}{2}[j(j+1)-l(l+1)-s(s+1)] \mid \Psi>
$$

com $j$ o números quânticos de momento angular total. Para (3.27), com $V$ sendo o potencial Coulombiano, $H_{S O C}$ toma a forma:

$$
H_{S O C}=\frac{a}{\hbar^{2}} \boldsymbol{S} \cdot \boldsymbol{L}
$$

onde $a$ é denominado constante de acoplamento de spin-órbita $a=\frac{\mu_{0} Z e^{2} \hbar^{2}}{8 \pi m_{e}^{2} r^{3}}$.

Sabe-se que

$$
<\frac{1}{r^{3}}>=\int \psi_{n, l, m}^{*} \frac{1}{r^{3}} \psi_{n, l, m} d \boldsymbol{r}=\left(\frac{Z \alpha m_{e} c}{\hbar}\right)^{3} \frac{2}{n^{3} l(l+1)(2 l+1)}
$$

com $\psi_{n, l, m}$ as funções de onda do átomo hidrogenoide, e $\alpha$ a constante de estrutura fina. Resulta que os níveis de energia do átomo hidrogenoide, com correções relativistas, toma a seguinte forma:

$$
E=E_{N R}+E_{S O C}=-\frac{1}{2} \frac{Z^{2}}{n^{2}}-\frac{\alpha^{2} Z^{4}}{2 n^{3}}\left(\frac{1}{j+1 / 2}-\frac{3}{4 n}\right)
$$


com $E_{N R}$ a energia não relativística e $n$ o número quântico principal. Para um elétron, $j$ pode assumir os valores de $l \pm \frac{1}{2}$

Energia para diferentes estados de um átomo hidrogenoide

\begin{tabular}{rrrrrrr} 
Estado & $\mathbf{n}$ & $\mathbf{l}$ & $\mathbf{s}$ & $\mathbf{j}$ & $E_{N R}\left[\frac{Z^{2}}{2}\right]$ & $E_{S O C}\left[\frac{\alpha^{2} Z^{4}}{2}\right]$ \\
\hline $1 s_{1 / 2}$ & 1 & 0 & $1 / 2$ & $1 / 2$ & 1 & $1 / 4$ \\
$2 s_{1 / 2}$ & 2 & 0 & $1 / 2$ & $1 / 2$ & $1 / 4$ & $5 / 64$ \\
$2 p_{1 / 2}$ & 2 & 1 & $-1 / 2$ & $1 / 2$ & $1 / 4$ & $5 / 64$ \\
$2 p_{3 / 2}$ & 2 & 1 & $1 / 2$ & $3 / 2$ & $1 / 4$ & $1 / 64$ \\
$3 s_{1 / 2}$ & 3 & 0 & $1 / 2$ & $1 / 2$ & $1 / 9$ & $9 / 324$ \\
$3 p_{1 / 2}$ & 3 & 1 & $-1 / 2$ & $1 / 2$ & $1 / 9$ & $9 / 324$ \\
$3 p_{3 / 2}$ & 3 & 1 & $1 / 2$ & $3 / 2$ & $1 / 9$ & $3 / 324$ \\
$3 d_{3 / 2}$ & 3 & 2 & $-1 / 2$ & $3 / 2$ & $1 / 9$ & $3 / 324$ \\
$3 d_{5 / 2}$ & 3 & 2 & $1 / 2$ & $5 / 2$ & $1 / 9$ & $1 / 324$
\end{tabular}

Tabela 3.1: Tabela dos níveis de energia para diferentes estados de um átomo hidrogenoide. $E_{N R}$ é a energia não relativística e $E_{S O C}$ a contribuição devido ao acoplamento spin-órbita (SOC). 


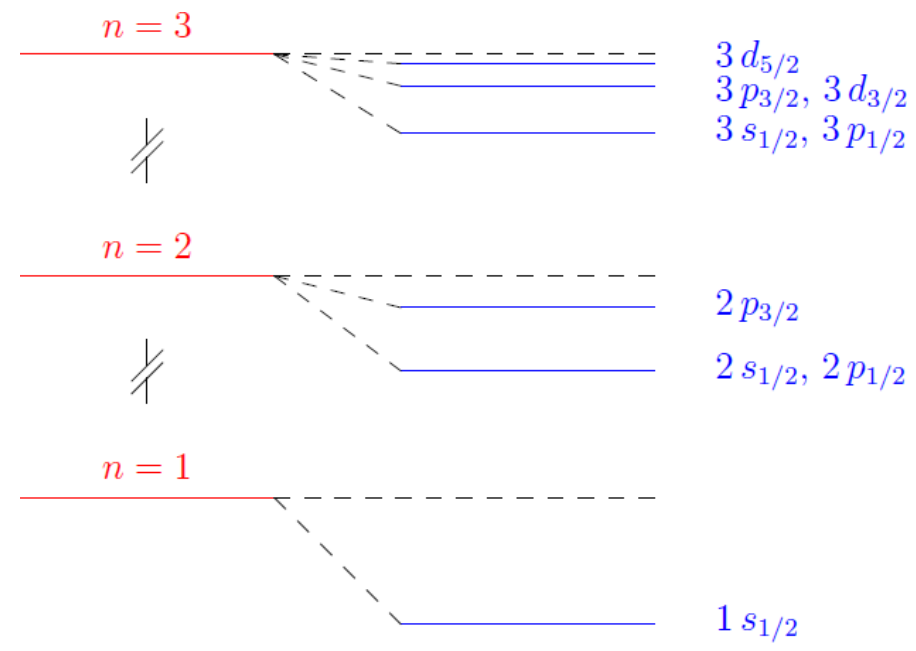

Figura 3.1: Ilustração do splitting devido ao acoplamento spin-órbita (SOC) para um átomo hidrogenoide. Sem SOC (à esquerda; somente depende do número quântico principal $n$ ) e com SOC (à direita depende de $n$ e do número quântico de momento angular total $j$ ).

\subsubsection{O acoplamento spin-órbita em estruturas cristalinas}

Diferente do caso atômico, para um cristal, os estados eletrônicos são configurados de tal forma que exista uma otimização das ligações químicas. Por exemplo, para uma rede cúbica simples são formados estados puramente $p_{x}, p_{y} \mathrm{e}$ $p_{z}$. Assim, a dispersão de energia em $\boldsymbol{k}$, para cada orbital atômico, dependerá do campo cristalino. Consequentemente, o SOC e o campo cristalino podem provocar cruzamentos adicionais nas estruturas de banda, dado que, existe a possibilidade que estados, que não sejam TRIM, apresentem cruzamentos acidentais devido às interações entre bandas. Entretanto, a forma como as bandas vão interagir será consequência da estrutura atômica do cristal.

Por outro lado, nos sistemas que não são invariantes por transformação de simetria de reversão temporal (TRS; time revarsal symmetry), a transformação $t \rightarrow-t$ muda o movimento de uma partícula do momento $k \mathrm{a}-k$. Outra propriedade que é afetada pela transformação de reversão temporal é a rotação intrínseca do elétron, e com isso, seu spin. A energia de um elétron ao se movimentar em uma direção com spin up é igual a de um elétron se movimentando na direção contraria 
com spin down, isto é:

$$
E(\boldsymbol{k}, \uparrow)=E(-\boldsymbol{k}, \downarrow)
$$

Contudo, para um material com simetria de inversão espacial, a operação $\boldsymbol{r} \rightarrow-\boldsymbol{r}$, não muda a rede cristalina. Assim,

$$
\begin{aligned}
& E(\boldsymbol{k}, \uparrow)=E(-\boldsymbol{k}, \uparrow) \\
& E(\boldsymbol{k}, \downarrow)=E(-\boldsymbol{k}, \downarrow) .
\end{aligned}
$$

Isto significa que a estrutura de bandas é simétrica com respeito ao centro da zona de Brillouin, $\boldsymbol{k}=0$. Também, que todas as bandas são bidegeneradas no spin. Materiais com esta propriedade são chamados de centrossimétricos.

Por outro lado, em materiais sem simetria de inversão, a degenerescência das bandas é quebrada como consequência do SOC e só (3.33) se mantém. Por exemplo, um sistema onde $V(\boldsymbol{r}) \neq V(-\boldsymbol{r})$ existirá uma diferença de potencial que dá lugar a um campo elétrico. Sabe-se que, elétrons ao se movimentar na presença de um campo elétrico, experimentam a presença de um campo magnético. E este, quebraria a simetria de reversão temporal, levando a:

$$
E(\boldsymbol{k}, \uparrow) \neq E(\boldsymbol{k}, \downarrow) .
$$

Percebe-se que o SOC assume diferente forma dependendo da estrutura do cristal e dos campos consequentes desta [26]. Isto foi observado pela primeira vez em 1955 por Dresselhaus [27], ele notou que em compostos semicondutores da família III-V quando carecem de um centro de inversão (BIA; Bulk invertion asymmetry), tais como GaAs ou InSb, o SOC $3 D$ próximo ao ponto $\Gamma$ possui um termo adicional da 
forma

$$
H_{D S O C}^{3 D} \propto p_{x}\left(p_{y}^{2}-p_{z}^{2}\right) s_{x}+p_{y}\left(p_{z}^{2}-p_{x}^{2}\right) s_{y}+p_{z}\left(p_{x}^{2}-p_{y}^{2}\right) s_{z}
$$

chamada hamiltoniana de Dresselhaus. Posteriormente, Bychkov e Rashba [11] reportaram um caso particular devido à falta de simetria de inversão estrutural (SIA; Structural inversion asymmetry) em heteroestruturas de GaAs/AlGaAs, onde a Hamiltoniana adicional ao SOC toma a forma

$$
H_{R} \propto\left(k_{x} \sigma_{y}-k_{y} \sigma_{x}\right)
$$

Estes termos são consequência do campo elétrico adicional, presente no cristal, devido à falta de simetria BIA ou SIA.

\subsubsection{O acoplamento spin-órbita Rashba}

O acoplamento spin-órbita Rashba ou efeito Rashba surge como consequência do SOC sobre sistemas com SIA. Por exemplo, uma superfície de $\mathrm{Au}(111)$, na qual a dispersão de energia pode ser descrita pelo modelo do elétron quase livre [28]. Neste caso, o elétron seria livre só em um plano. Tomando $V=V_{\perp}$ como o potencial que confina os elétrons ao sólido no plano $x, y$. Ao considerar o SOC, o potencial leva a $\nabla V=d V / d z \boldsymbol{e}_{\boldsymbol{z}}$ onde $z$ é o eixo normal à superfície, e $\boldsymbol{e}_{\boldsymbol{z}}$ é um vetor unitário apontando na direção z. Neste modelo, o gradiente do potencial essencialmente controla a força do SOC. O SOC resultante assume a forma:

$$
H_{R}=\alpha_{R}\left(\boldsymbol{e}_{\boldsymbol{z}} \times \boldsymbol{p}\right) \cdot \boldsymbol{\sigma}=\alpha_{R}\left(k_{x} \sigma_{y}-k_{y} \sigma_{x}\right)
$$

chamado de acoplamento spin-órbita Rashba ou efeito Rashba. $\alpha_{R}$ é o parâmetro (ou constante) de Rashba. A solução ao problema $H=\frac{\hbar^{2} \boldsymbol{k}^{2}}{2 m^{*}}+H_{R}$ é simples e pode ser encontrada analiticamente. O resultado é ilustrado através da figura (3.2): 


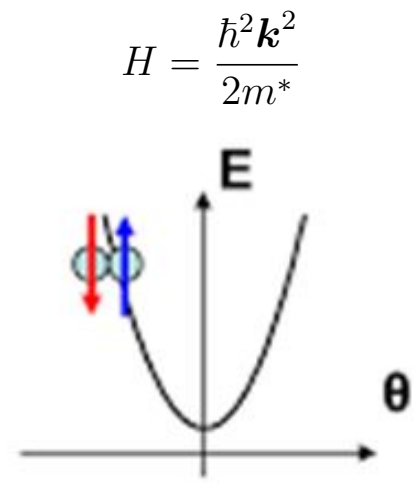

a) Dispersão de energia do modelo do elétron quase livre.

$$
H=\frac{\hbar^{2} \boldsymbol{k}^{2}}{2 m^{*}}+\alpha_{R}\left(k_{x} \sigma_{y}-k_{y} \sigma_{x}\right)
$$
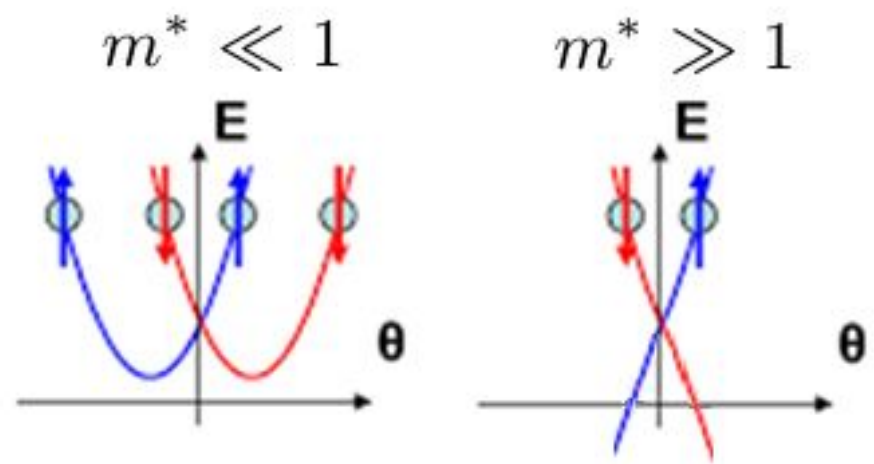

b) Dispersão de energia do modelo do elétron quase livre com efeito Rashba.

Figura 3.2: Ilustração do spin splitting devido ao efeito Rashba para um gás de elétrons livres. a) Sem SOC b) Esquerda: splitting para pequenas contribuições do efeito Rashba. Direita: splitting para grandes contribuições do efeito Rashba, também chamado "Giant Rashba spin splitting".

A falta de simetria de inversão, além da diferença de potencial em $z$, poderia dar origem a contribuições de ordem maior para o efeito Rashba [29]. Este efeito, acopla o spin com o momento linear e quebra a degenerescência no spin para dar lugar a duas sub-bandas de energia como observado na figura (3.2b). Materiais com este comportamento têm sido caraterizados experimentalmente em superfícies formadas com átomos pesados, tais como $\mathrm{Bi}$ e $\mathrm{Pb}$ [30,31]. Também reportaram materiais que apresentam um efeito Rashba de maior pronunciamento (figura 3.2b direita) os quais são chamados de "efeito Rashba gigante" ( "Giant Rashba effect" 
ou "Giant Rashba spin splitting").

\subsubsection{Efeito Hall de spin e Hall quântico de Spin}

O efeito Hall de spin (SHE; Spin Hall Effect) e Hall quântico de spin (QSH; Quantum Spin Hall) são consequência da interação de spin-órbita. Estes efeitos podem ser gerados por uma variedade de mecanismos de acoplamento spinórbita intrínsecos ou extrínsecos. Uma vez que o acoplamento spin-órbita dá origem à dependência dos movimentos orbitais com o spin do elétron, o spin pode ser manipulado por meios puramente elétricos [32,33]. Por exemplo, uma corrente elétrica perpendicular a um metal 2D gera uma corrente de spin transversal. Esta induz uma acumulação de spins opostos nas bordas do material. Este efeito é conhecido como efeito Hall de spin e é ilustrado na figura (3.3).

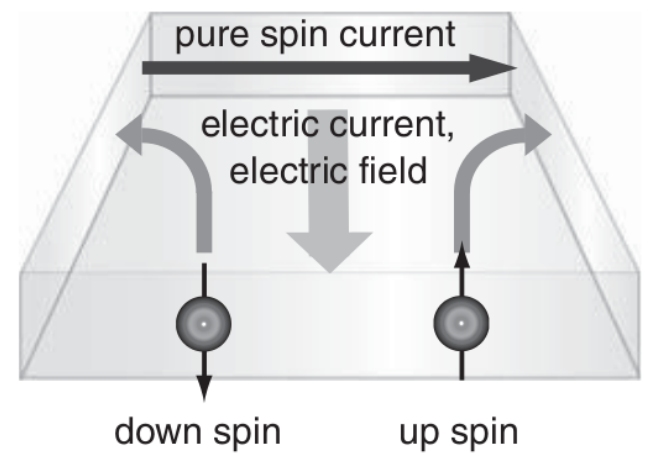

Figura 3.3: Ilustração do efeito Hall de spin [34].

No SHE, previsto teoricamente em 1971 por Dyakonov e Perel [35], as correntes de borda são polarizadas de spin devido ao seu acúmulo gerado pela corrente elétrica de espalhamento no bulk. Uma vez a corrente elétrica no bulk é invertida, as orientações do spin dos estados de borda também são invertidas.

O efeito QSH ${ }^{1}$ pode ser ilustrado como um gás de elétrons bidimensional com um campo magnético forte. Neste caso, os elétrons formam níveis de Landau

\footnotetext{
${ }^{1} \mathrm{O}$ efeito Hall quântico de spin é a versão "quântica" do efeito Hall de spin, análogo a como o efeito Hall quântico é a versão quântica do efeito Hall [34].
} 
e a energia de Fermi $E_{F}$ está entre estes níveis. Assim, não existem estados de bulk entorno de $E_{F}$, sendo o bulk isolante. No entanto, entorno de $E_{F}$, existem alguns outros estados que estão localizados na borda do sistema. Estes estados, chamados estados de borda, apresentam quiralidade, isto significa que, eles percorrem a borda apenas em uma direção. No efeito Hall quântico (QHE), o número de estados de borda $N$ é um invariante topológico e independe dos detalhes da borda. $N$ é chamado de número de Chern e determina a condutividade Hall quantizada $\sigma_{x y}=N e^{2} / h$.

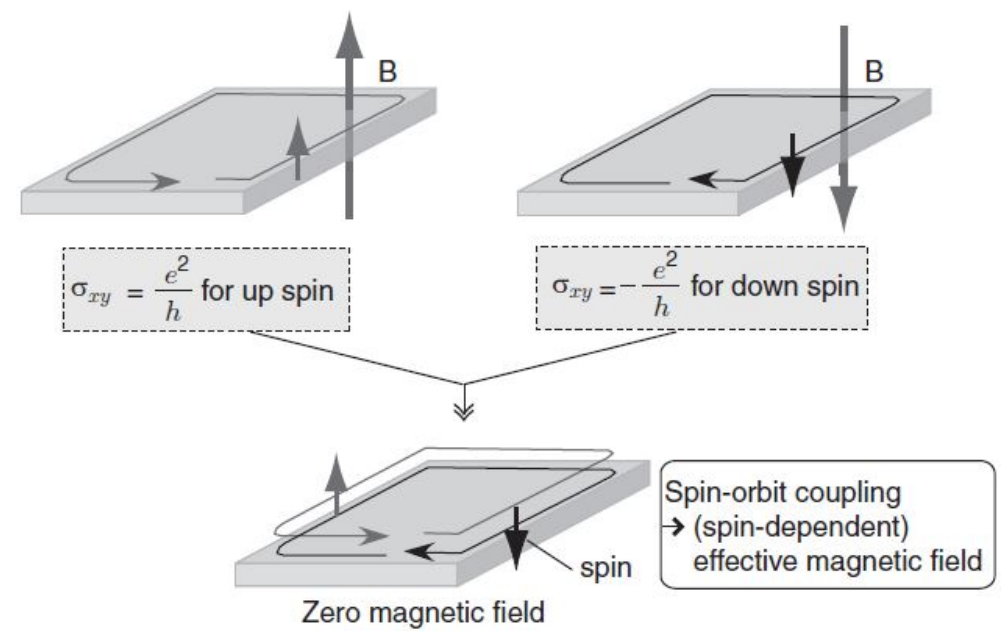

Figura 3.4: Ilustração da construção de um sistema com efeito Hall quântico de spin (isolante topológico) como uma superposição de dois subsistemas com efeito Hall de spin, um com topologia $N=1 \mathrm{com}$ spin up e outro com topologia $N=-1 \mathrm{com}$ spin down [34].

Os sistemas que apresentam efeito QSH são chamados de isolantes topológicos. Estes, podem ser esquematizados conforme a figura (3.4). Ao sobrepor dois subsistemas: i) o sistema de elétrons com spin-up sob o campo magnético $+B$ cuja condutividade Hall é $\sigma_{x y}=N e^{2} / h$ (número de Chern $N=1$ ), e ii) o sistema de elétrons com spin-down sob o campo magnético $-B$ com $\sigma_{x y}=N e^{2} / h$ (número de Chern $N=-1$ ). Para realizar este sistema como um todo, é necessário aplicar dois campos magnéticos simultaneamente com signos contrários. Se ambos forem extrínsecos, resultá em um campo magnético nulo. Entretanto, é possível realizá-lo pelo acoplamento spin-órbita intrínsecos em sólidos. Os estados resultantes teriam spins opostos, propagando-se em direções contrárias, em cada bordas. Estes estados 
de borda são estados próprios de $s_{z}$, ou seja, os spins dos estados de borda são perpendiculares ao plano 2D. Porém, não é uma condição necessária em isolantes topológicos $2 \mathrm{D}$.

Ao considerar a direção do spin dos estados de borda, assume-se a simetria de reversão temporal. Dado que, esta simetria implica que o estado de borda que se movimenta no sentido horário, é equivalente ao que se move no sentido anti-horário após uma inversão temporal. Esta transformação afeta tanto ao momento linear como ao spin. Os estados de borda são degenerados devido ao teorema de Kramers, e são chamados de pares de Kramers. Estes são topologicamente protegidos, e são robustos sob toda perturbação que preservem a simetria de reversão temporal (como impurezas não magnéticas, interação elétron-elétron, etc). Esta proteção deve-se ao número topológico, proveniente da caracterização topológica da estrutura de banda.

Os isolantes são caracterizados pelo invariante topológico Z2 [36-38], o qual pode tomar dois valores, 0 e 1 (também chamados de "par" e "ímpar" ou "trivial" e "não trivial"). Quando Z2 é par, corresponde a um isolante trivial (3.5a). Se for ímpar, corresponde a um isolante topológico (3.5b). Estes últimos, tem são observados em sistemas bidimensionais (2D) com estados metálicos de borda com quiralidade e tridimensionais (3D) com estados metálicos de superfície com helicidade.

\section{2 $\mathrm{Bi}_{2} \mathrm{Se}_{3}$ e Família de Isolantes Topológicos (3D)}

Dentre os isolantes topológicos (TI) mais estudados estão a família de TI tridimensionais (3D) romboédricos $B i_{2} S e_{3}, B i_{2} T e_{3}$ e $S b_{2} T e_{3}$, propostos teoricamente em 2007 por Kane e Fu [39], confirmado via cálculo de primeiros princípios por Zhang em 2009 [23] e caracterizado experimentalmente por Hasan em 2009 [40]. 

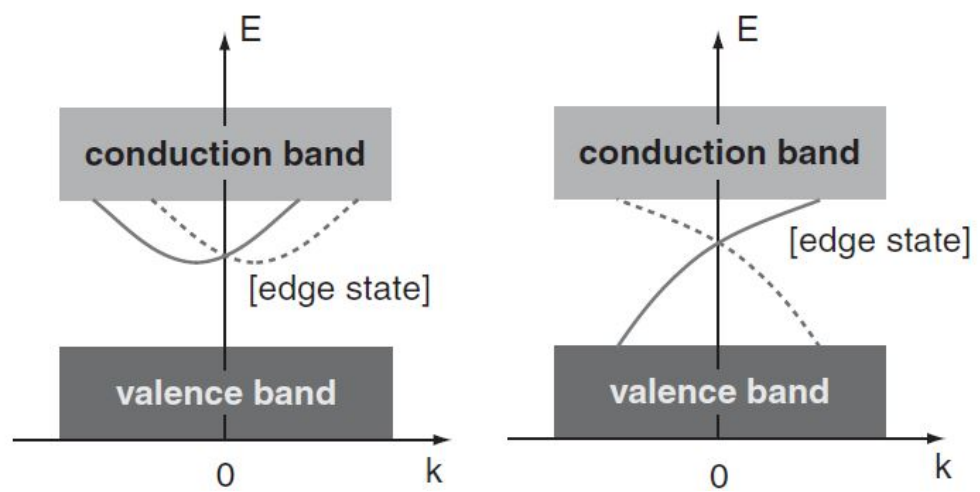

Figura 3.5: Ilustração dos estados de borda de isolantes 2D. a) Isolante trivial e b) Isolante topológico. As linhas sólidas e tracejadas denotam os estados de borda (um para cada borda) com spin opostos e velocidades opostas, devido à simetria de reversão temporal. [34].

Para ir do estado de isolante trivial (sem inversão de banda) para o isolante não trivial (com banda invertida) é necessário passar por um estado metálico. Consequentemente, são manifestados estados metálicos na superfície destes sistemas. Estes estados metálicos formam dois cones de Dirac (DC) bidegenerados, localizados no centro da Zona de Brillouin (ponto $\Gamma$ ). $\mathrm{O} \mathrm{Bi}_{2} \mathrm{Se}_{3}$, diferente dos outros TI 3D romboédricos, apresenta na sua superfície os cones de Dirac "mais limpo" no em torno do nível de Fermi.

Ter um cone de Dirac mais limpo é importante desde o ponto de vista de cálculo das propriedades de transporte, dado que, só os estados que formam o cone de Dirac são levados em conta no cálculo de transmitância, evitando "ruídos" por espalhamentos indesejados. Assim, cone de Dirac limpos são mais promissores para aplicações de spintrônica. Quando existem outros estados entorno $E_{F}$, estes podem contribuir no espalhamento, dificultando o controle do spin. Vários trabalhos tem sido focados com o objetivo de obter DC mais limpos [41,42].

Quando o SOC é considerado, no bulk do $B i_{2} S e_{3}$ aparece um gap não trivial no ponto $\Gamma$. Este gap é de aproximadamente $0.3 \mathrm{eV}$, o qual é maior que a escala de energia da temperatura ambiente, pelo que é esperado que mudanças de temperatura no ambiente não quebrem o estado topológico deste sistema. 


\subsubsection{Propriedades estruturais}

Os TI $\mathrm{Bi}_{2} \mathrm{Se}_{3}, \mathrm{Bi}_{2} \mathrm{Te}_{3}$ e $S b_{2} \mathrm{Te}_{3}$ possuem estrutura cristalina romboédrica do grupo espacial $D_{3 d}^{5}(R \overline{3} m)$. Estes, possuem cinco camadas atômicas na sua célula unitária, que pertencem a três grupos de camadas que também contém cinco átomos chamados de Quintuple Layers (QLs) conforme pode ser observado na figura (3.6a).

a)

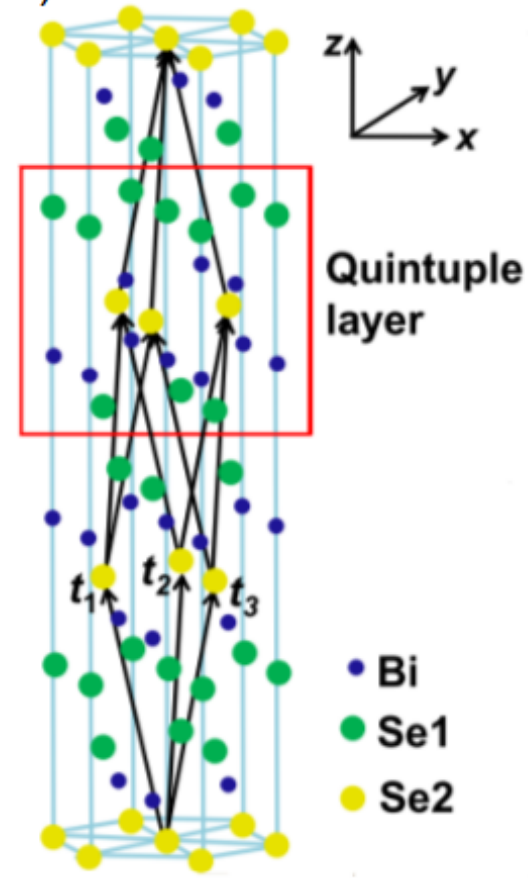

b)

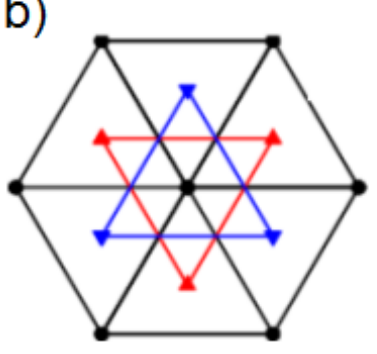

Sítio $A$ Sítio B $\nabla$ Sítio $C$

C)

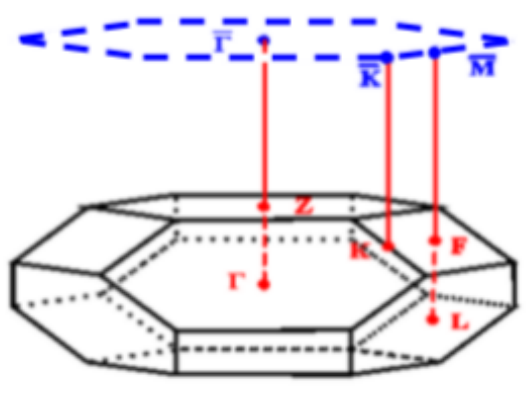

Figura 3.6: a) Supercélula hexagonal do $\mathrm{Bi}_{2} \mathrm{Se}_{3}$, com 15 camadas atômicas (3 QLs). Os vetores primitivos da célula unitária são representados por $t_{1}, t_{2}$ e $t_{3}$. b) Recorte no eixo $z$ da rede triangular, onde é observado três diferentes tipos de sítios não equivalentes. c) Primeira zona de Brillouin que contém 4 pontos TRIM (TimeReversal-Invariant-Momentum) e a superfície 2D (projeção da zona em 3D) da zona de Brillouin que está representada em linhas azuis. Imagem da referência [23].

O cristal é formado por empilhamentos de QLs ao longo da direções $z$. As ligações químicas entre átomos Bi e Se são do tipo covalente-iônica e as ligações entre QLs são de tipo Van der Waals [43].

Os vetores primitivos são dados por: 


$$
\begin{aligned}
& t_{1}=\left(\frac{-a}{2}, \frac{-\sqrt{3} a}{6}, \frac{c}{3}\right) \\
& t_{2}=\left(\frac{a}{2}, \frac{-\sqrt{3} a}{6}, \frac{c}{3}\right) \\
& t_{3}=\left(0, \frac{\sqrt{3} a}{3}, \frac{c}{3}\right)
\end{aligned}
$$

com $a$ e $c$ parâmetros da rede na célula hexagonal, cujos valores estão definidos na tabela 3.2.

\begin{tabular}{l|rrr} 
& $\mathrm{Sb}_{2} \mathrm{Te}_{3}$ & $\mathrm{Bi}_{2} \mathrm{Te}_{3}$ & $\mathrm{Bi}_{2} \mathrm{Se}_{3}$ \\
\hline$a(\AA)$ & 4.250 & 4.383 & 4.138 \\
$c(\AA)$ & 30.35 & 30.487 & 28.64 \\
\hline
\end{tabular}

Tabela 3.2: Valores dos parâmetros, a, c para diferentes elementos da família de isolantes topológicos $D_{3 d}^{5}$.

Cada QL é formada por um arranjo de átomos no formato de camadas com estrutura triangular, onde são intercalados camadas de átomos de Bi e Se na sequência ... -Se1-Bi-Se2-Bi-Se1- ..., onde Se1 e Se2 representam dois átomos não equivalentes de Se no cristal ( figura 3.6a). Na figura (3.6b) mostra uma vista superior dos sítios das correspondentes camadas atômicas não equivalentes.

O bulk do Bi2Se3 é formado por um número infinito de QLs, com três tipos diferentes de QL denotadas como QL1, QL2 y QL3, com rotação de $60^{\circ}$ entre elas e empilhadas sequencialmente da forma ...-QL1-QL2-QL3-QL1-QL2-QL3-... para formar o bulk pristino. A estrutura cristalina do bulk dá lugar a uma correspondente zona de Brillouin 3D com geometria ortorrômbica (figura 3.6c desenhada por linhas pretas) a qual tem 4 TRIM não equivalentes $\Gamma(0,0,0), \mathrm{L}(1 / 2,0,0), \mathrm{F}(1 / 2,1 / 2$ , 0) e $Z(1 / 2,1 / 2,1 / 2)$, escritos em função dos vetores da rede recíproca que são 
definidos como:

$$
\begin{aligned}
& s_{1}=\frac{1}{h}\left(-1, \frac{-\sqrt{3}}{3}, b\right) \\
& s_{2}=\frac{1}{h}\left(1, \frac{-\sqrt{3}}{3}, b\right) \\
& s_{3}=\frac{1}{h}\left(0,2 \frac{\sqrt{3}}{3}, b\right)
\end{aligned}
$$

A zona de Brillouin 2D é uma projeção da 3D no eixo $z$, conforme o desenho em azul da figura (3.6c). Os pontos $\Gamma$ e $Z$ da zona 3D são projetados em $\bar{\Gamma}$. L e F são projetados em $\bar{M}$. Além do que, tem-se o ponto $\bar{K}$, onde este último não é um TRIM. Os vetores da rede cristalina da superfície são definidos como:

$$
\begin{aligned}
t_{1}^{\text {sup }} & =(a, 0,0) \\
t_{2}^{\text {sup }} & =\left(\frac{a}{2}, \frac{\sqrt{3} a}{2}, 0\right) \\
t_{3}^{\text {sup }} & =\left(0,0, \frac{c}{2}\right)
\end{aligned}
$$

Para reproduzir a superfície do $\mathrm{Bi}_{2} \mathrm{Se}_{3}$ nos códigos computacionais tais como SIESTA ou VASP, é necessário fornecer o parâmetro $c=c^{\prime}+c_{\text {vacuo }}$, no qual $c^{\prime}$ é a largura dada pelo número de QLs consideradas na superfície e $c_{v a c u o}$, o tamanho do vácuo adicionado com o fim de evitar a interação entre as superfícies replicadas ${ }^{2}$. O uso de $c_{\text {vacuo }} \geq 10 \AA$ é suficiente para evitar a interação entre as superfícies replicadas.

\footnotetext{
${ }^{2}$ As replicas das superfícies surgem devido a que os códigos VASP e SIESTA são implementados para usar condições periódicas de contorno.
} 


\subsubsection{Propriedades eletrônicas}

Na figura (3.7) mostra-se de forma esquemática os níveis atômicos do Bi e Se sujeitos a diferentes campos. A evolução dos níveis atômicos para o ponto $\Gamma$ surge ao considerar: ligações químicas, simetria de inversão, campo cristalino, e interação spin-órbita. O orbital $p_{z}$ proveniente do átomo de $\mathrm{Bi}\left(\left|\mathrm{P} 1_{-}^{+}, \pm 1 / 2\right\rangle\right)$, inverte com o orbital $p_{z}$ do átomo de Se $\left(\left|\mathrm{P} 2_{+}^{-}, \pm 1 / 2\right\rangle\right)$. É bem conhecido que neste sistema só acontece uma única inversão de banda, a qual tem lugar no ponto $\Gamma$. Um número ímpar de pontos na zona de Brillouin cria um gap na fase bulk e este é chamado de gap não trivial ou gap invertido.

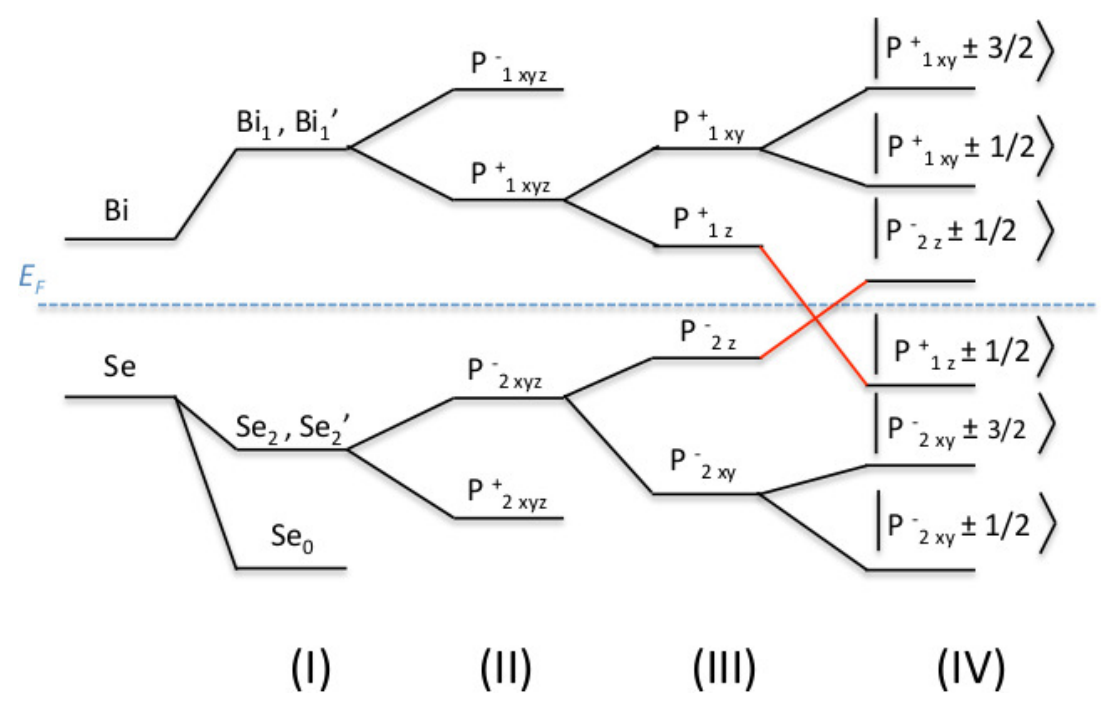

Figura 3.7: Representação esquematica da inversão de banda dada no ponto $\Gamma$ para o bulk do sistema $\mathrm{Bi}_{2} \mathrm{Se}_{3}$. A figura mostra a evolução dos orbitais atômicos $p_{x, y, z}$ do Bi e Se quando são considerados i) os efeitos das ligações químicas mais ii) a formação dos estados ligantes e antiligantes dada pela simetria de inversão mais iii) o campo cristalino e iv) interação de spin-órbita (SOC). Nota-se que existe uma inversão de bandas entre os estados $p_{z}$ do Bi e do Se quando o SOC é levada em conta [23].

Na dispersão da bandas de energia do bulk $B i_{2} S e_{3}$ (figura 3.8) é mostrada a projeção nos orbitais $p_{z}$ do Bi e Se. Quando não é considerado o SOC (figura 3.8a), os estados que formam o topo da banda de valência são formados majoritariamente por $p_{z}$ do Se enquanto o fundo da banda de condução está formada predominantemente por $p_{z}$ do Bi. Quando o SOC é considerado (figura 3.8b) se da lugar a um splitting 


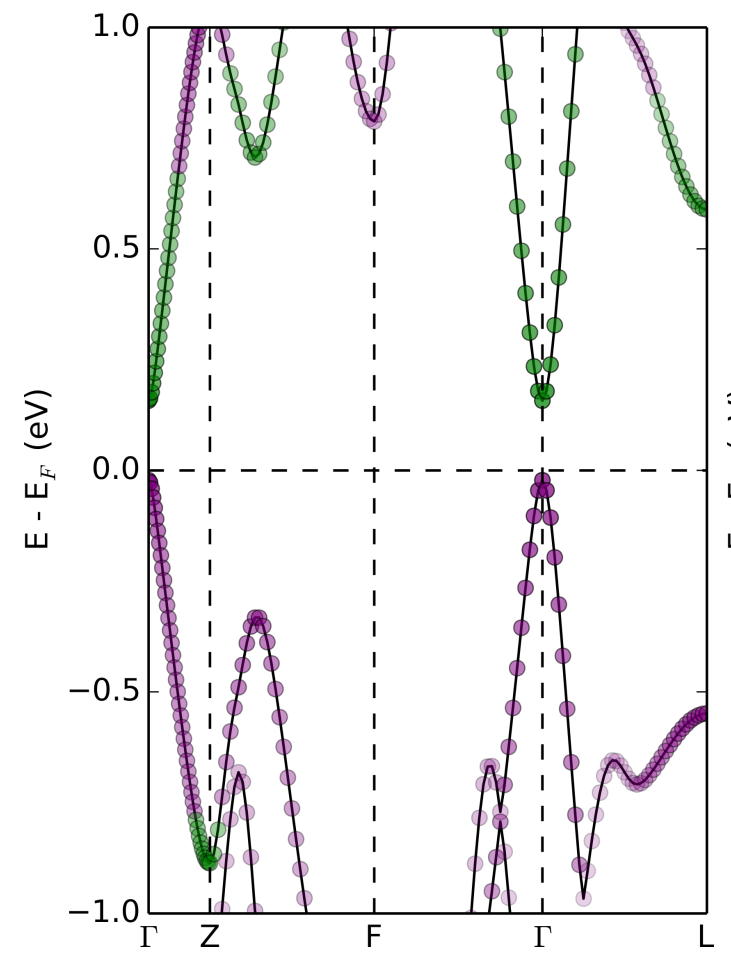

(a) Estrutura de banda sem SOC

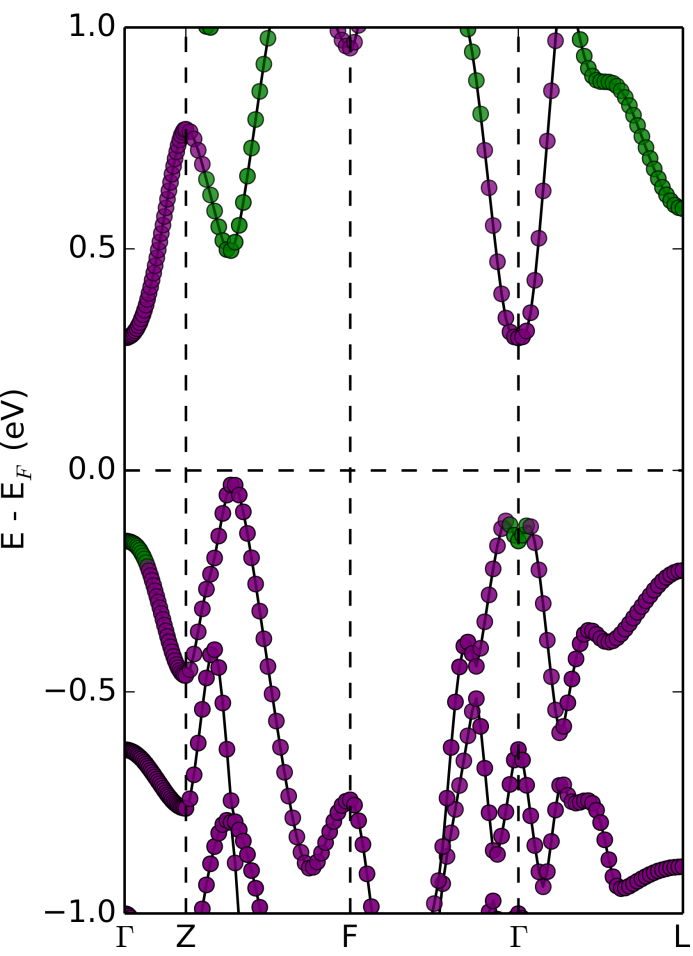

(b) Estrutura de banda com SOC

Figura 3.8: Estrutura de banda do bulk do sistema $B i_{2} S e_{3}$ ao longo do caminho $\Gamma \rightarrow Z \rightarrow F \rightarrow \Gamma \rightarrow L$. a) Sem acoplamento spin-órbita (SOC). b) Com SOC. Em ambas são representadas as projeções dos orbitais $p_{z}$ do $\mathrm{Bi}$ (verde) e do Se (roxo) [44].

entre os estados. Este splitting causa uma inversão entre os estados no ponto $\Gamma$ levando a um gap não trivial de $0.3 \mathrm{eV}$.

$\mathrm{Na}$ imagem (3.9) evidencia-se a estrutura eletrônica da superfície para diferentes números de QLs. Em (3.9a) mostra-se a dispersão das bandas de energia sem SOC para a superfície de 1-6 QLs. Nota-se que, sem SOC o sistema não apresenta estados metálicos de superfície. $\mathrm{Na}(3.9 \mathrm{~b})$ as dispersões de energia correspondem ao mesmos sistemas, porém, com SOC. Nos casos para 1-3 QLs, o sistema apresenta gap de energia e, para um número maior a 4 QLs, o sistema é metálico. A falta de estados metálicos para um número pequeno de QLs devese aos estados de ambas superfícies interagir entre si, dado pela proximidade das superfícies, o que impossibilita a formação dos canais de condutividade. 

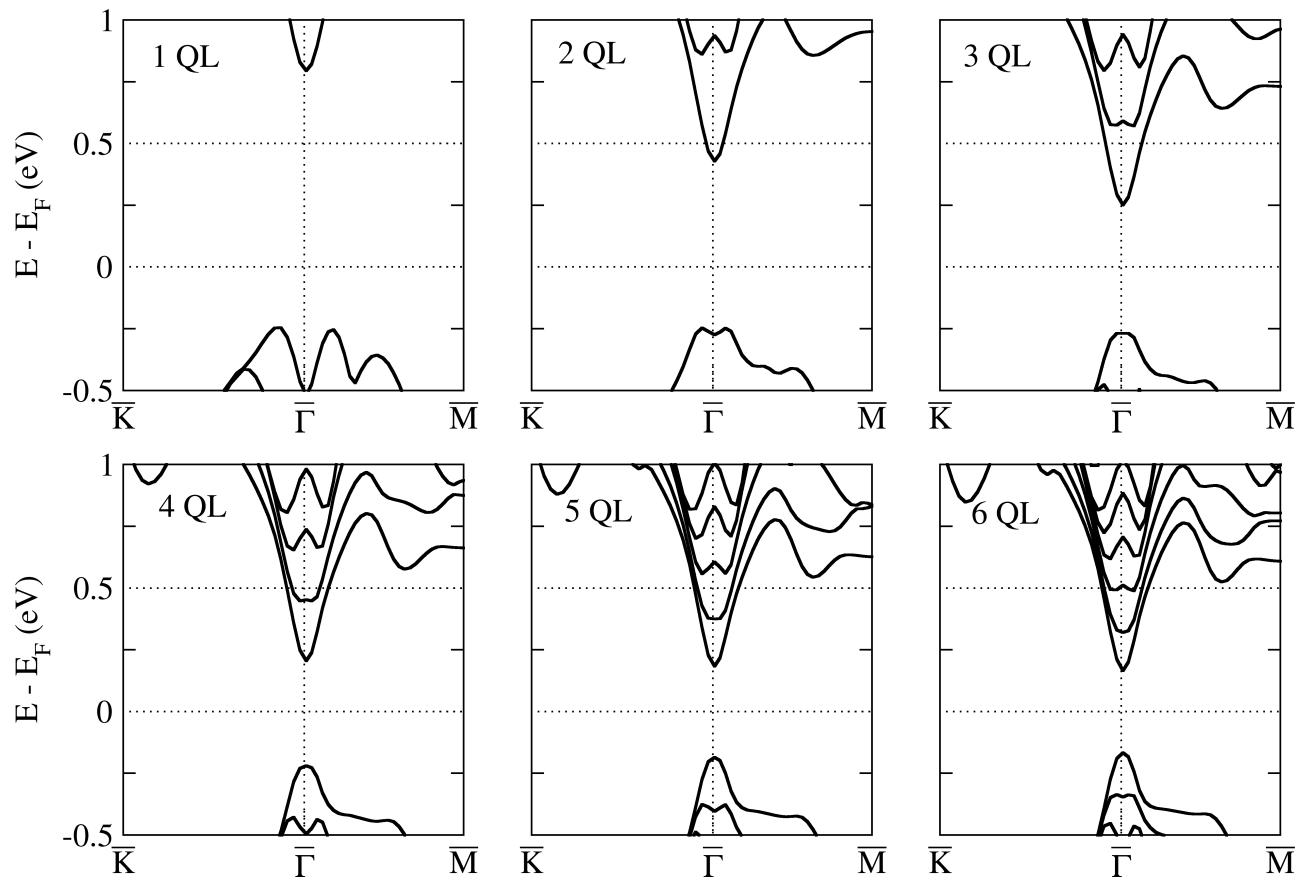

a) Estrutura de banda sem SOC
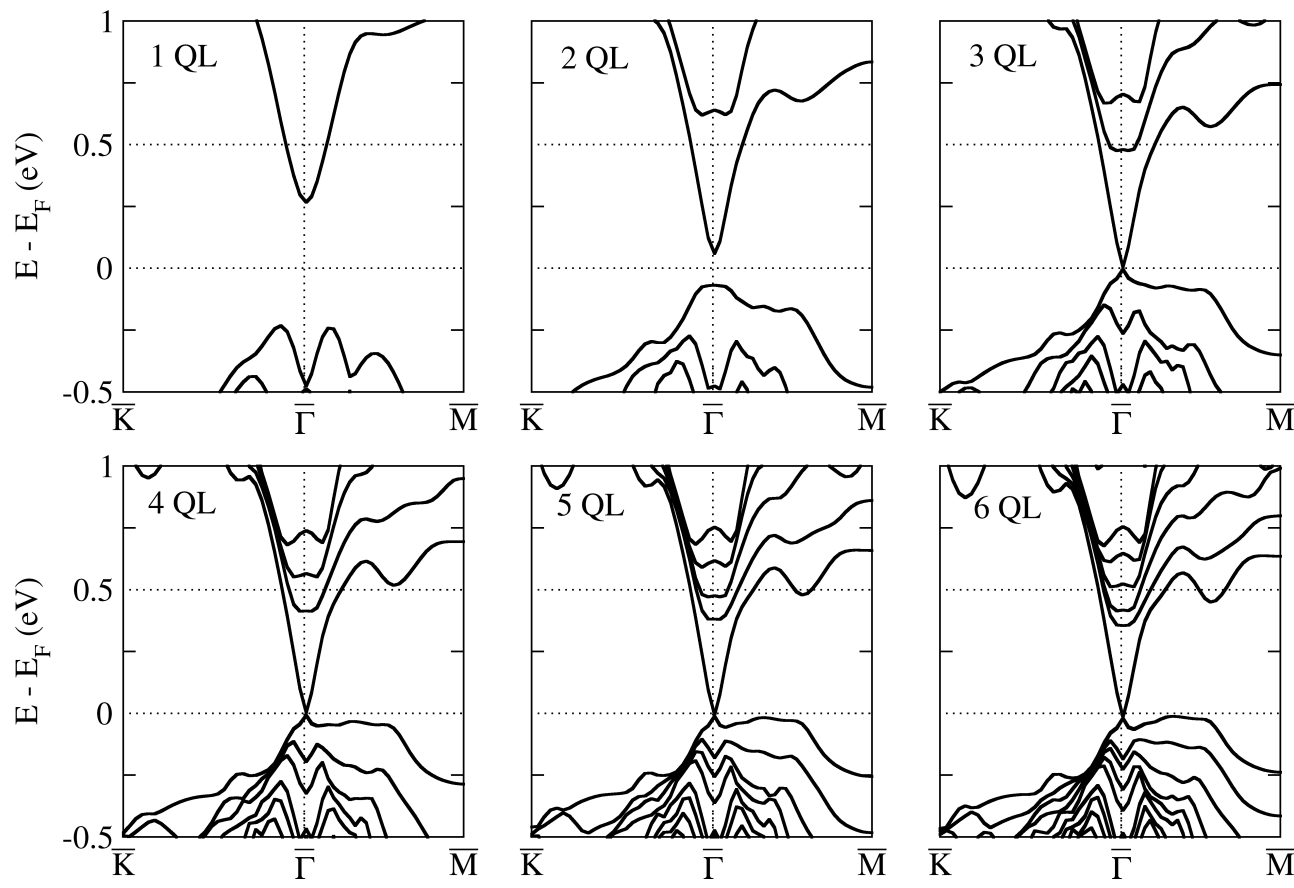

b) Estrutura de banda com SOC

Figura 3.9: Estrutura de banda da superfície do sistema $B i_{2} S e_{3}$ para 1-6 quintuple layer (QL) ao longo do caminho $\bar{K} \rightarrow \bar{\Gamma} \rightarrow \bar{M}$; a) Sem acoplamento spin-órbita (SOC); b) Com SOC [44]. 

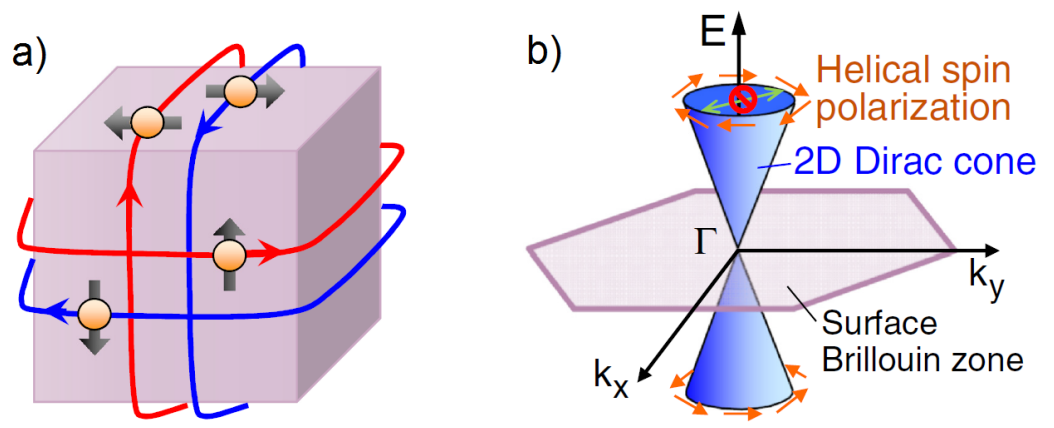

Figura 3.10: Representação ilustrativa dos estados de superfície de um isolante topológico (TI). a) Esquematização dos canais de condutividade com helicidade no spin formados no espaço real de um TI 3D. b) Dispersão de energia dos estados na superfície de um 3D TI que formam o cone de Dirac 2D. O spin é representado pelas setas. O spin é não degenerado, possuem helicidade e retroespalhamento proibido [45].

Os estados metálicos formados na superfície apresentam uma dispersão tipo Dirac. Isto é, para o limite $k \rightarrow \Gamma$, a dispersão é linear. A degenerescência do spin é quebrada nos estados de superfície o que provoca helicidade no spin. Isto impede o retroespalhamento entre os estados de superfície (figura 3.10b).

As propriedades eletrônicas e de transporte nestes sistemas também podem ser estudadas por modelos efetivos. Zhang reportou um modelo efetivo baseado em propriedades de simetria [23]. Este modelo permite estudar os diferentes sistemas $B i_{2} \mathrm{Se}_{3}, \mathrm{Bi}_{2} \mathrm{Te}_{3}$ e $S b_{2} \mathrm{Te}_{3}$, através do ajuste de parâmetros.

\subsubsection{O modelo de Zhang}

Nesta seção são apresentadas as propriedades eletrônicas da superfície do $\mathrm{Bi}_{2} \mathrm{Se}_{3}$ obtidas pelo modelo de Zhang [46]. Primeiramente é apresentado o modelo do bulk proposto por Zhang. Posteriormente serão consideradas condições abertas de contorno para introduzir o efeito de tamanho finito e reproduzir a superfície, como feito por Tanaka [47].

Na figura (3.9) foram evidenciadas as estruturas eletrônicas obtidas por 
cálculos ab initio para a superfície do $\mathrm{Bi}_{2} \mathrm{Se}_{3}$. Estas podem ser estudadas através de um modelo simples com custo computacional relativamente baixo. Para uma base de 4 autoestados, toda hamiltoniana $4 \times 4$ pode ser expandida com as matrizes de Dirac como:

$$
H_{e f f}=\epsilon(\boldsymbol{k}) \mathbf{1}_{\mathbf{4} \times \mathbf{4}}+\sum_{i} d_{i}(\boldsymbol{k}) \Gamma_{i}+\sum_{i j} d_{i j}(\boldsymbol{k}) \Gamma_{i j}
$$

no qual $\mathbf{1}_{\mathbf{4} \times \mathbf{4}}$ é a matriz identidade, $\Gamma_{i}(i=1 \ldots 5)$ são as 5 matrizes de Dirac

$$
\begin{gathered}
\Gamma_{1}=\left(\begin{array}{llll}
0 & 0 & 0 & 1 \\
0 & 0 & 1 & 0 \\
0 & 1 & 0 & 0 \\
1 & 0 & 0 & 0
\end{array}\right), \Gamma_{2}=\left(\begin{array}{cccc}
0 & 0 & 0 & -i \\
0 & 0 & -i & 0 \\
0 & i & 0 & 0 \\
i & 0 & 0 & 0
\end{array}\right), \Gamma_{3}=\left(\begin{array}{cccc}
0 & 1 & 0 & 0 \\
1 & 0 & 0 & 0 \\
0 & 0 & 0 & -1 \\
0 & 0 & -1 & 0
\end{array}\right) \\
\Gamma_{4}=\left(\begin{array}{cccc}
0 & -i & 0 & 0 \\
i & 0 & 0 & 0 \\
0 & 0 & 0 & -i \\
0 & 0 & i & 0
\end{array}\right), \Gamma_{5}=\left(\begin{array}{cccc}
1 & 0 & 0 & 0 \\
0 & -1 & 0 & 0 \\
0 & 0 & 1 & 0 \\
0 & 0 & 0 & -1
\end{array}\right)
\end{gathered}
$$

que satisfazem $\left\{\Gamma_{i}, \Gamma_{j}\right\}=2 \delta_{i j}$. Por sua parte, $\Gamma_{i j}$ são os dez comutadores das matrizes definidos como $\Gamma_{i j}=\left[\Gamma_{i}, \Gamma_{j}\right] / 2 i$.Entretanto, $\epsilon(\boldsymbol{k}), d_{i}(\boldsymbol{k})$, e $d_{i j}(\boldsymbol{k})$ são polinômios expandidos em potências de $\boldsymbol{k}$.

As matrizes $\Gamma$ e as funções expandidas como potencias de $\boldsymbol{k}$ são somadas dependendo da representação às quais pertencem. Para determinar a representação destas, são construídos os operadores de simetria do grupo puntual do sistema.

Considerando cada QL como uma rede triangular 2D onde cada sítio na rede corresponde a uma QL da célula unitária do $\mathrm{Bi}_{2} \mathrm{Se}_{3}$. Esta representação preserva a simetria do grupo pontual $D_{3 d}^{5}(R 3 m)$, que possui: 
1. Simetria de rotação $R_{3}$ ao longo do eixo $z$

2. Simetria de rotação $R_{2}$ ao longo do eixo $x$

3. Simetria de inversão (paridade) $\mathcal{P}$

4. Simetria de reversão temporal $\mathcal{T}$.

Na figura (3.7) foi esquematizado que os estados relevantes na descrição da fase topológica (estados que levam à inversão de banda) são $\left|\mathrm{P} 1_{-}^{+}, 1 / 2\right\rangle,\left|\mathrm{P} 2_{+}^{-}, 1 / 2\right\rangle$, $\left|\mathrm{P} 1_{-}^{+},-1 / 2\right\rangle$ e $\left|\mathrm{P} 2_{+}^{-},-1 / 2\right\rangle$. Estes estados obedecem às transformações de simetria quando os operadores de transformação são definidos como:

1. $R_{3}=e^{i(\Pi / 2) \theta} \operatorname{com} \prod=\sigma_{3} \otimes 1_{2 \times 2}$ e $\theta=2 \pi / 3$

2. $R_{2}=i \sigma_{1} \otimes \tau_{3}$

3. $\mathcal{P}=1_{2 \times 2} \otimes \tau_{3}$

4. $\mathcal{T}=\Theta K$ onde $\Theta=i \sigma_{2} \otimes 1_{2 \times 2}$ e $K$ é o operador complexo conjugado.

Sendo $\sigma$ a base que atua sob o spin e $\tau$ a dos estados que formam a sub-banda $P 1^{+}$ e $P 2^{-}$. Baseado nestes operadores de transformação, são obtidas as representações para cada matriz $\Gamma_{i}$ e para as potências de $\boldsymbol{k}$ (Tabela 3.3).

É esperado que a simetrias do cristal e de reversão temporal sejam preservadas. Consequentemente, devem ser escolhidos as matrizes $\Gamma_{i}$ e os polinômios de $\boldsymbol{k}$ com a mesma representação. Por exemplo, $\Gamma_{1}$ e $\Gamma_{2}$ levam à representação $\tilde{\Gamma}_{3}^{-}$ e são ímpar (-) sob inversão temporal, assim, são associados a $k_{x}$ e $k_{y}$ para formar um termo invariante na Hamiltoniana.

Desconsiderando os termos de terceira ordem em $k$ ou maiores, obtém-se a hamiltoniana:

$$
H_{0}=\epsilon(\boldsymbol{k})+\mathcal{M}(\boldsymbol{k}) \Gamma_{5}+\mathcal{B}\left(\boldsymbol{k}_{\boldsymbol{z}}\right) \Gamma_{4} k_{z}+\mathcal{A}(\boldsymbol{k})\left(\Gamma_{1} k_{y}-\Gamma_{2} k_{x}\right),
$$




\begin{tabular}{lcc}
\hline & Representação & $\mathcal{T}$ \\
\hline$\left\{\Gamma_{1}, \Gamma_{2}\right\}$ & $\tilde{\Gamma}_{3}^{-}$ & - \\
$\Gamma_{3}$ & $\tilde{\Gamma}_{1}^{-}$ & - \\
$\Gamma_{4}$ & $\tilde{\Gamma}_{2}^{-}$ & - \\
$\Gamma_{5}$ & $\tilde{\Gamma}_{1}^{+}$ & + \\
$\Gamma_{12}$ & $\tilde{\Gamma}_{2}^{+}$ & - \\
$\left\{\Gamma_{23}, \Gamma_{31}\right\}$ & $\tilde{\Gamma}_{3}^{+}$ & - \\
$\left\{\Gamma_{14}, \Gamma_{24}\right\}$ & $\tilde{\Gamma}_{3}^{+}$ & - \\
$\left\{\Gamma_{15}, \Gamma_{25}\right\}$ & $\tilde{\Gamma}_{3}^{-}$ & + \\
$\Gamma_{34}$ & $\tilde{\Gamma}_{2}^{+}$ & - \\
$\Gamma_{35}$ & $\tilde{\Gamma}_{1}^{-}$ & + \\
$\Gamma_{45}$ & $\tilde{\Gamma}_{2}^{-}$ & + \\
$\left\{k_{x}, k_{y}\right\}$ & $\tilde{\Gamma}_{3}^{-}$ & - \\
$k_{z}, k_{z}^{3}$ & $\tilde{\Gamma}_{2}^{-}$ & - \\
$1, k_{x}^{2}+k_{y}^{2}, k_{z}^{2}$ & $\tilde{\Gamma}_{1}^{+}$ & + \\
$\left\{k_{x}^{2}-k_{y}^{2}, 2 k_{x} k_{y}\right\}$ & $\tilde{\Gamma}_{3}^{+}$ & + \\
$k_{x}^{3}-3 k_{x} k_{y}^{2}$ & $\tilde{\Gamma}_{1}^{-}$ & - \\
$3 k_{x}^{2} k_{y}-k_{y}^{3}$ & $\tilde{\Gamma}_{2}^{-}$ & - \\
$\left\{k_{x}^{3}+k_{x} k_{y}^{2}, k_{x}^{2} k_{y}+k_{y}^{3}\right\}$ & $\tilde{\Gamma}_{3}^{-}$ & - \\
\hline
\end{tabular}

Tabela 3.3: Representações para as matrizes $\Gamma$ e os polinômios de $\boldsymbol{k}$ [46] .

onde $\epsilon(\boldsymbol{k})=C_{0}+C_{1} k_{z}^{2}+C_{2}\left(k_{x}^{2}+k_{y}^{2}\right), \mathcal{M}(\boldsymbol{k})=M_{0}+M_{1} k_{z}^{2}+M_{2}\left(k_{x}^{2}+k_{y}^{2}\right)$, $\mathcal{A}(\boldsymbol{k})=A_{0}+A_{2}\left(k_{x}^{2}+k_{y}^{2}\right)$ e $\mathcal{B}\left(\boldsymbol{k}_{\boldsymbol{z}}\right)=B_{0}+B_{2} k_{z}^{2}$. Com $C_{i}, M_{i}, A_{i}$ e $B_{i}$ parâmetros ajustáveis conforme o sistema. Os termos $A_{2}$ e $B_{2}$ representam correção de ordem maior para a velocidade de Fermi $A_{0}$ e $B_{0}$ não são importantes no entorno do ponto $\Gamma(\boldsymbol{k} \approx 0)[23]$. Explicitamente, a Hamiltoniana é escrita como

$$
\mathcal{H}=\varepsilon(\boldsymbol{k})+\left(\begin{array}{cccc}
\mathcal{M}(\vec{k}) & 0 & B_{0} k_{z} & A_{0} k_{-} \\
0 & \mathcal{M}(\vec{k}) & A_{0} k_{+} & -B_{0} k_{z} \\
B_{0} k_{z} & A_{0} k_{-} & -\mathcal{M}(\boldsymbol{k}) & 0 \\
A_{0} k_{+} & -B_{0} k_{z} & 0 & -\mathcal{M}(\boldsymbol{k})
\end{array}\right),
$$

$\operatorname{com} k_{ \pm}=k_{x} \pm i k_{y}$

Seguindo o enfoque apresentado por Tanaka [47]. Introduz-se a transformação $k_{i} a_{i} \rightarrow \sin \left(k_{i} a_{i}\right), \quad\left(k_{i} a_{i}\right)^{2} \rightarrow 2\left(1-\cos \left(k_{i} a_{i}\right)\right)$, por considerar a 
aproximação para $k_{i}$ pequenos, com $a_{1}=a_{2}=a, a_{3}=c$ os parâmetros da rede. O Hamiltoniano do bulk é reescrito como

$$
\mathcal{H}=\tilde{\varepsilon}(\boldsymbol{k})+\left(\begin{array}{cccc}
\tilde{\mathcal{M}}(\vec{k}) & 0 & \bar{B}\left(k_{z}\right) & \bar{A}_{-} \\
0 & \tilde{\mathcal{M}}(\vec{k}) & \bar{A}_{+} & -\bar{B}\left(k_{z}\right) \\
\bar{B}\left(k_{z}\right) & \bar{A}_{-} & -\tilde{\mathcal{M}}(\boldsymbol{k}) & 0 \\
\bar{A}_{+} & -\bar{B}\left(k_{z}\right) & 0 & -\tilde{\mathcal{M}}(\boldsymbol{k})
\end{array}\right)
$$

com

$$
\begin{gathered}
\bar{B}\left(k_{z}\right)=\frac{B_{0}}{c} \sin \left(k_{z}\right), \\
\varepsilon(\boldsymbol{k})=C_{0}+\frac{2 C_{1}}{c^{2}}\left(2-\cos k_{z}^{2}\right)+\frac{2 C_{2}}{a^{2}}\left(1-\cos k_{x}^{2}-\cos k_{y}^{2}\right), \\
\mathcal{M}(\boldsymbol{k})=M_{0}+\frac{2 M_{1}}{c^{2}}\left(2-\cos k_{z}^{2}\right)+\frac{2 M_{2}}{a^{2}}\left(1-\cos k_{x}^{2}-\cos k_{y}^{2}\right), \\
\bar{A}_{ \pm}\left(k_{x}, k_{y}\right)=\frac{A_{0}}{a}\left(\sin k_{x} a \pm i \sin k_{y} a\right) .
\end{gathered}
$$

O Hamiltoniano do bulk (3.46) é modificado para considerar um número finito de QLs. Para a interação dos primeiros vizinhos mais próximos na rede tetragonal, a Hamiltoniana da superfície consiste de um termo inter-layer $\mathcal{H}_{z}$ e um termo intra-layer $\mathcal{H}_{0}$, dado por:

$$
\mathcal{H}_{\text {layer }}=\sum_{n=1}^{N_{q l}} \boldsymbol{c}_{n}^{\dagger} \mathcal{H}_{0} \boldsymbol{c}_{n}+\sum_{n=1}^{N_{q l}-1}\left[\boldsymbol{c}_{n}^{\dagger} \mathcal{H}_{z} \boldsymbol{c}_{n+1}+\text { c.c. }\right]
$$

no qual $N_{\mathrm{QL}}$ é o número de QLs e determina a espessura da layer. $c_{n}^{\dagger}\left(c_{n}\right)$ os correspondentes operadores de criação (aniquilação) e c.c. denota complexo 
conjugado. O termo intra-layer seria

$$
\mathcal{H}_{0}=\varepsilon_{0}(\boldsymbol{k})+\left(\begin{array}{cccc}
\mathcal{M}_{0}(\vec{k}) & 0 & 0 & \bar{A}_{-} \\
0 & \mathcal{M}_{0}(\vec{k}) & \bar{A}_{+} & 0 \\
0 & \bar{A}_{-} & -\mathcal{M}_{0}(\boldsymbol{k}) & 0 \\
\bar{A}_{+} & 0 & 0 & -\mathcal{M}_{0}(\boldsymbol{k})
\end{array}\right) \text {, }
$$

onde $\varepsilon_{0}(\boldsymbol{k})=C_{0}+\frac{2 C_{1}}{c^{2}}+\frac{C_{2}}{a^{2}}\left(2-\cos k_{x}^{2}-\cos k_{y}^{2}\right)$ e $\mathcal{M}_{0}(\boldsymbol{k})=M_{0}+\frac{2 M_{1}}{c^{2}}+\frac{M_{2}}{a^{2}}(2-$ $\left.\cos k_{x}^{2}-\cos k_{y}^{2}\right)$. E o termo inter-layer

$$
\mathcal{H}_{z}=\left(\begin{array}{cccc}
-\frac{M_{1}+C_{1}}{a^{2}} & 0 & i \bar{B} / c & 0 \\
0 & -\frac{M_{1}+C_{1}}{a^{2}} & 0 & -i \bar{B} / c \\
i \bar{B} / c & 0 & \frac{M_{1}-C_{1}}{a^{2}} & 0 \\
0 & -i \bar{B} / c & 0 & \frac{M_{1}-C_{1}}{a^{2}}
\end{array}\right)
$$

o qual fornece o acoplamento entre as QLs consecutivas.

O estado topológico da superfície do $\mathrm{Bi}_{2} \mathrm{Se}_{3}$ aparece explicitamente quando é considerada a superfície com um número de QLs maior ou igual a quatro. Os estados de superfície possuem uma dispersão linear entorno do ponto $\Gamma$ formando o conde de Dirac.

Usando os parâmetros propostos por Zhang, $M_{0}=-0.28 \mathrm{eV}, M_{1}=0.216$ $\mathrm{eV}, A_{0}=0.80 \mathrm{eV}, B_{0}=0.32 \mathrm{eV}, C_{0}=-0.0083 \mathrm{eV}, C_{1}=0.024 \mathrm{eV}, C_{2}=1.77 \mathrm{eV}$, $a=4.14 \AA$, e $c=9.55 \AA$, obtemos os resultados apresentados na figura (3.11) para diferentes números de QLs.

No próximo capitulo serão abordadas as propriedades eletrônicas e estruturais do $\mathrm{Bi}_{2} \mathrm{Se}_{3}$ quando impurezas de $\mathrm{Sn}$ são depositadas na superfície. Esta deposição quebra a simetria de inversão estrutural, porém, espera-se que o estado topológico permaneça, dado que o Sn é um átomo não magnético e não induziria polarização de spin no sistema. 

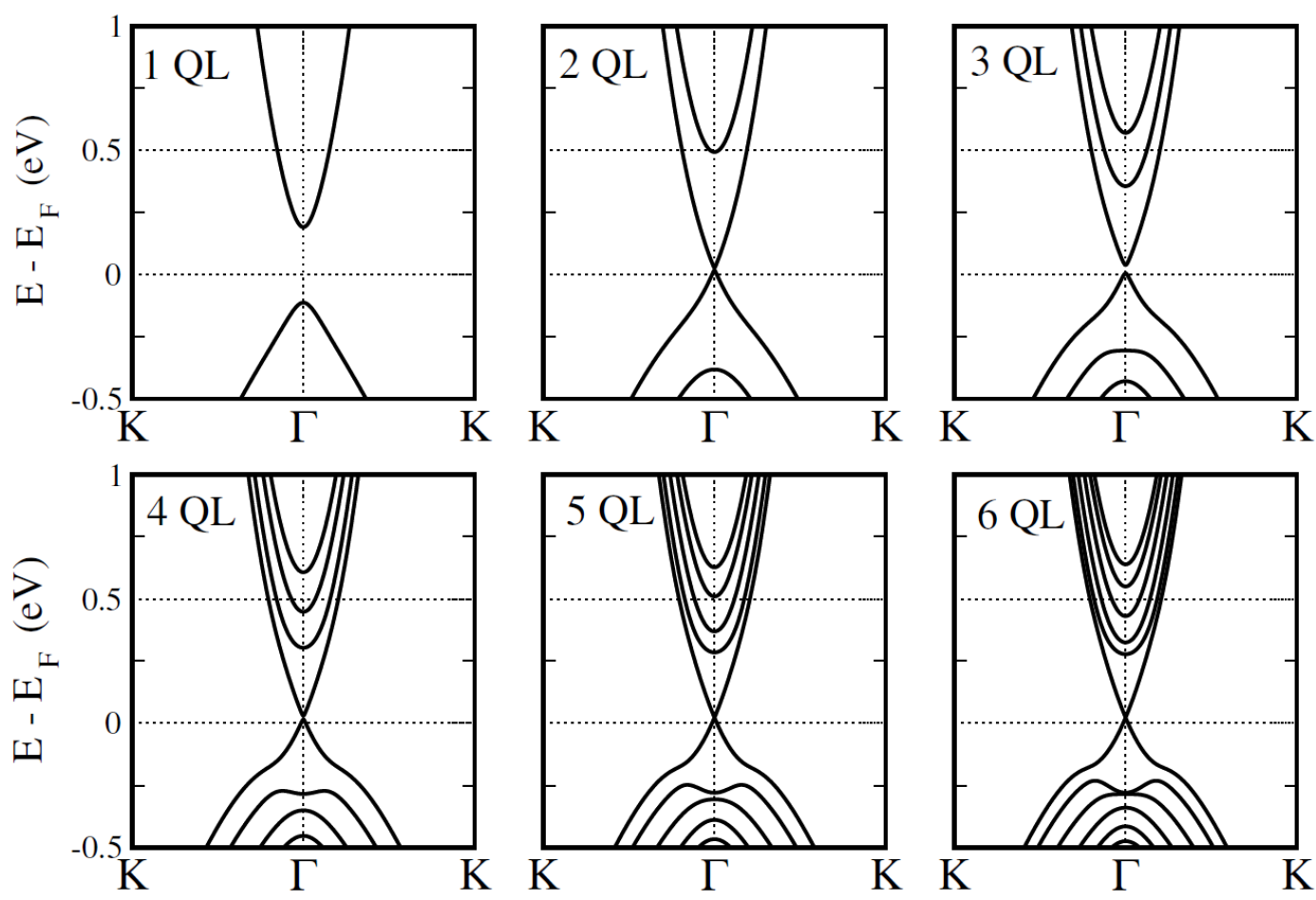

Figura 3.11: Dispersão de energia dado pelo modelo de Zhang [23] dos estados de bulk e superfície cerca do ponto $\Gamma$ nos filmes finos de $B i_{2} S e_{3}$ formados por 1-6 QLs. 


\section{Parte II}

\section{Resultados}




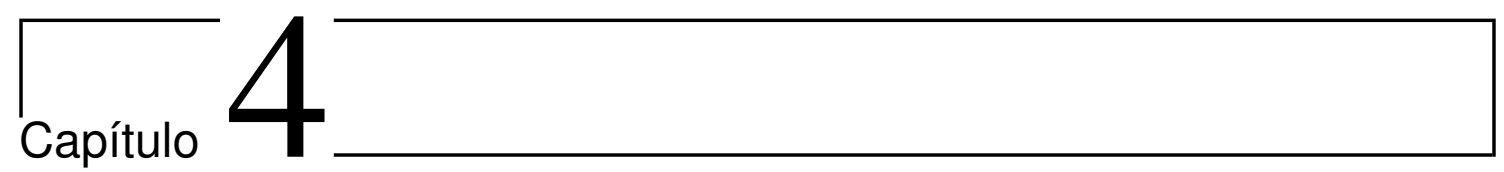

\section{Sn adsorvido na superfície do $\mathrm{Bi}_{2} \mathrm{Se}_{3}$}

Devido ao grande interesse nas propriedades estruturais e eletrônicas dos isolantes topológicos, é investigado como mudam tais propriedades com a deposição de diferentes impurezas. Por exemplo, ao dopar com metais de transições como Fe, Mn, e Cr, são encontrados: Fases ferromagnéticas no $B i_{2} T e_{3}$ [48]; Efeito Hall quântico anômalo nos TI romboédricos [49]; Supercondutividade no $\mathrm{Bi}_{2} \mathrm{Se}_{3}$ com $\mathrm{Cu}$ intercalado [50,51]; Fase ferromagnética com alta mobilidade no $\mathrm{Sm} / \mathrm{Bi}_{2} \mathrm{Se}_{3}$. Entretanto, ao dopar com impurezas não magnéticas foi encontrado: Supercondutividade induzida por efeitos de proximidade em interfaces de $\mathrm{Sn} / \mathrm{Bi}_{2} \mathrm{Se}_{3}$ [52]; Crescimento de estaneno sobre uma superfície do $B i_{2} \mathrm{Te}_{3}$ [53]; Entre outros. Neste capitulo reportamos que no sistema $\mathrm{Bi}_{2} \mathrm{Se}_{3}$ dopado com Sn, existe uma fase interticial, a qual, é energeticamente favorável.

Também investigamos como são modificadas as propriedades eletrônicas da superfície do $\mathrm{Bi}_{2} \mathrm{Se}_{3}$ quando é quebrada a simetria de inversão estrutural. Propomos o modelo modificado de Zhang para reproduzir tais resultados. Encontramos que, sobre a superfície pode ser formado um terceiro cone de Dirac como consequência do efeito Rashba e da mudança de massa efetiva no sistema. Este terceiro cone, também localizado no ponto $\Gamma$, apresenta simetria de reversão temporal e textura 
de spin helicoidal.

Os resultados são obtidos com cálculo de primeiros princípios de acordo com a Teoria do Funcional da Densidade (DFT), como implementado no código SIESTA. O código SIESTA usado aqui foi modificado em um trabalho prévio [54] onde é incluído o SOC seguindo a aproximação on-site [55]. Para descrever o funcional de troca-correlação é usado a aproximação da densidade local. Primeiramente apresentamos os resultados obtidos com cálculos Ab-initio e logo comparamos estes com os obtidos no modelo efetivo.

\subsection{Propriedades estruturais do $\mathrm{Sn} / \mathrm{Bi}_{2} \mathrm{Se}_{3}$}

A superfície usada para realizar a deposição é formada com um arranjo de 4 QLs, sobre a QL superior são localizados os sítios (T1,T2 e T3 conforme mostra-se na figura 4.1) onde inicialmente é realizada a deposição. As posições T1 T2 e T3 estão localizada com iguais componentes x,y dos átomo Se1, Bi, e Se2 respectivamente, porém, com componente z (altura) $1.5 \AA$ acima da camada Se1. Também foi estudada outra posição discriminada como T3in ou intersticial, localizada com iguais componentes x,y que T3 e com a mesma altura que a camada Se1.

Em uma supercélula com periodicidade $2 \times 2$ são localizados 4 sítios equivalentes para cada posição. Assim, é possível estudar o mapa de estabilidade energético para diferentes porcentagens de recobrimento. As concentrações do recobrimento são discriminadas como 100\%, $75 \%$ 50\% e $25 \%$ quando quatro, três, dois ou um sítios respectivamente, estão ocupados por uma impureza.

A energia de adsorção por átomo de Sn foi calculado de acordo com: 

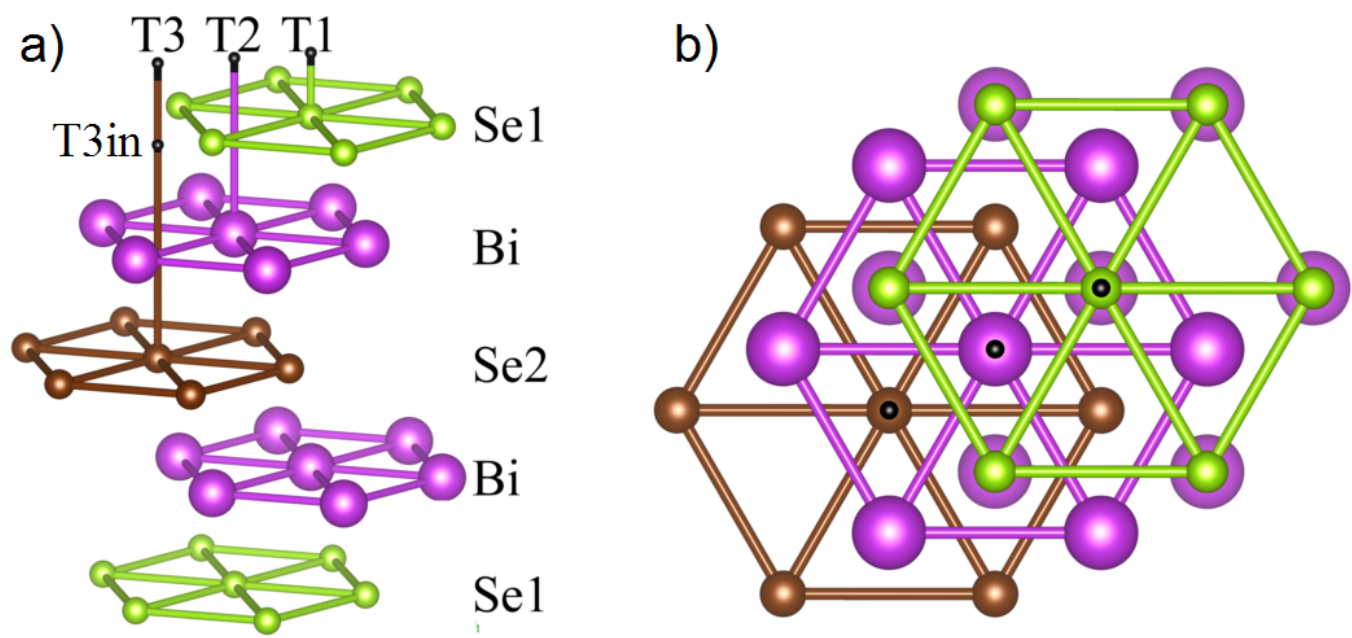

Figura 4.1: Estrutura cristalina do $S n / B i_{2} S e_{3}$, a) Vista lateral da QL superior discriminando as posições T1-3 b) Vista superior da geometria do $S n / B i_{2} S_{3}$.

$$
E b=\frac{E_{n}\left[S n / B i_{2} S e_{3}\right]-E\left[B i_{2} S e_{3}\right]-n \cdot E[S n]}{n}
$$

onde $n$ é o número de átomos de $S n$ na base, $E\left[B i_{2} S e_{3}\right]$ é a energia total do $B i_{2} S e_{3}$ pristino, $E[S n]$ é a energia para um átomo de $S n$ isolado e $E_{n}\left[S n / B i_{2} S e_{3}\right]$ a energia do sistema $S n / B i_{2} S e_{3}$ com $n$ átomos de $\mathrm{Sn}$ adsorvidos na superfície do $B i_{2} S e_{3}$.

\begin{tabular}{lcc}
\hline \hline \multicolumn{1}{c}{ Sítio } & Recobrimento & Eb $(\mathrm{eV})$ \\
\hline T1 & $100 \%$ & -2.676 \\
T2 & $100 \%$ & -2.632 \\
T3 & $100 \%$ & -2.640 \\
T3in & $100 \%$ & -2.798 \\
T2 & $75 \%$ & -2.736 \\
T3 & $75 \%$ & -2.698 \\
T2 & $50 \%$ & -2.655 \\
T3 & $50 \%$ & -2.952 \\
T2 & $25 \%$ & -2.414 \\
T3 & $25 \%$ & -2.468 \\
\hline
\end{tabular}

Tabela 4.1: Energia de adsorção por átomo depositado no respetivo sítio.

A tabela (4.1) resume os resultados obtidos. Nenhum dos casos produz polarização magnética no sistema. Observa-se que para o caso T3 com uma concentração de $50 \%$ há uma energia de ligação maior, o que favorece este sítio para 
ocorrer a deposição. Depois de relaxar o sistema com a impureza nos determinados sítios, os átomos de Sn ficaram com a mesmas componentes x,y que da posição inicial e com uma altura $1.8 \AA$ acima (tomando como referência a altura da camada de Se1).

Em todos os casos, as impurezas ficaram acima da camada de Se1, exceto para o sítio T3in 100\%. Neste caso os átomos de Sn apresentam uma maior tendência a ficarem próximos dos Bi. Isto obriga aos Se1 a saírem da sua posição inicial, causando uma surfactação na superfície. Observada esta tendência, primeiramente é estudada a possibilidade do sistema T3in ser formado experimentalmente por métodos como IBAD (íon beam assisted deposition), Deposição por sputtering, etc. Para isso, estimamos a barreira de energia necessária para colocar os átomos de Sn na posição T3in. A barreira de energia foi calculada com 180 passos estruturais, sendo o passo inicias é onde os átomos de Sn estão em uma posição o suficientemente afastada da superfície como para não haver ligação. O passo final corresponde com a estrutura do sistema relaxado T3in (figura 4.2). Observa-se que, parametrizando a energia em função da posição com respeito à camada Se1, a energia total do sistema apresenta um máximo para $h=0$, sendo $h=($ altura do Sn) - (Altura do Se1). Este máximo é esperado, dado que, para $h=0$ os átomos de Sn encontra-se na mesma altura que os Se1, causando um stress no sistema devido à pouca separação entre os Sn e os Se1. A barreira estimada foi aproximadamente de $3.93 \mathrm{eV}$. Este valor é muito alto para ser superada em condições não extremas. Entretanto, após vencida o sistema é estável, com uma energia de adsorção exotérmica. Por exemplo, o sistema poderia ser criado por molecular beam epitaxy [56] de modo a evitar a barreira de potencial. 


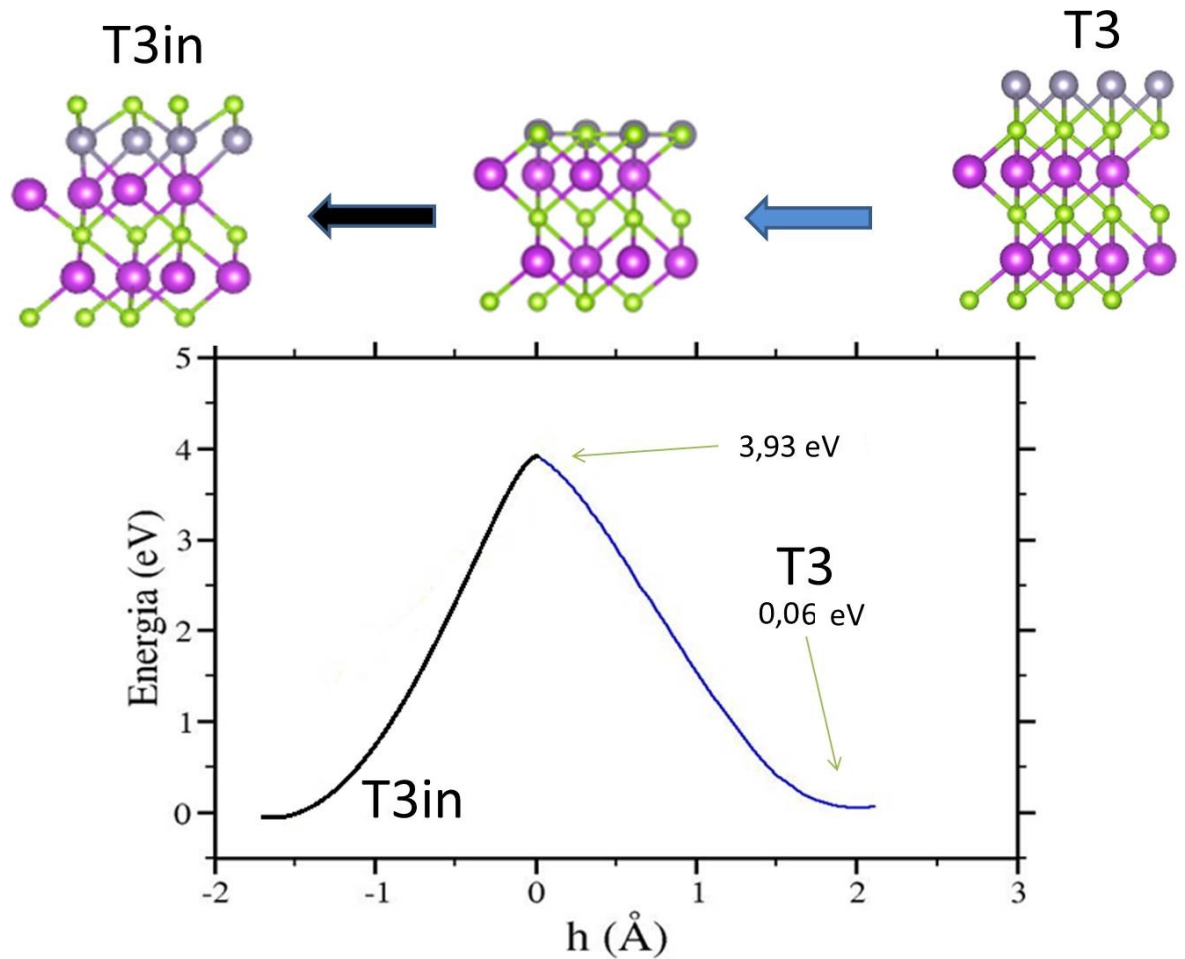

Figura 4.2: Barreira de potencial existente para passar da posição T3 a T3in no caso de um recobrimento de $100 \%$. A energia do sistema T3 é $\approx 0.06 \mathrm{eV}$ maior que o T3in. $\mathrm{h}=$ (altura do Sn) - (Altura do Se1).

\subsection{Propriedades eletrônicas do $\mathrm{Sn} / \mathrm{Bi}_{2} \mathrm{Se}_{3}$}

A estrutura eletrônica obtidas para os sistemas com 100\% de recobrimento são mostrados na figura (4.3). Pode-se observar que os sistemas continuam apresentando uma fase metálica. Em torno do nível de energia $-0.4 \mathrm{eV}$ é observada uma dispersão linear nas vizinhanças do ponto $\Gamma$, similar ao caso pristino, isto indica que a fase topológica esta sendo mantida.

No caso T3in são observadas 3 dispersões lineares para os níveis -0.38, -0.30 e $0.0 \mathrm{eV}$ no ponto $\Gamma$, as quais chamaremos de cone 1 , cone 2 , e cone 3 respectivamente. A origem destes cones são investigadas por cálculo de dispersão de bandas com projeção nos orbitais atômicos. Na figura (4.4) é apresentada a estrutura de bandas, na qual são projetados os orbitais dos átomos das superfícies. Os orbitais representados em verde (vermelho) estão localizados na superfície inferior 
T1

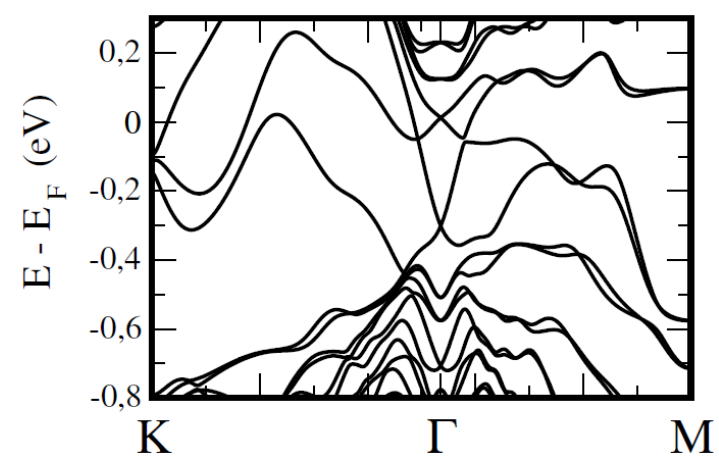

T3in

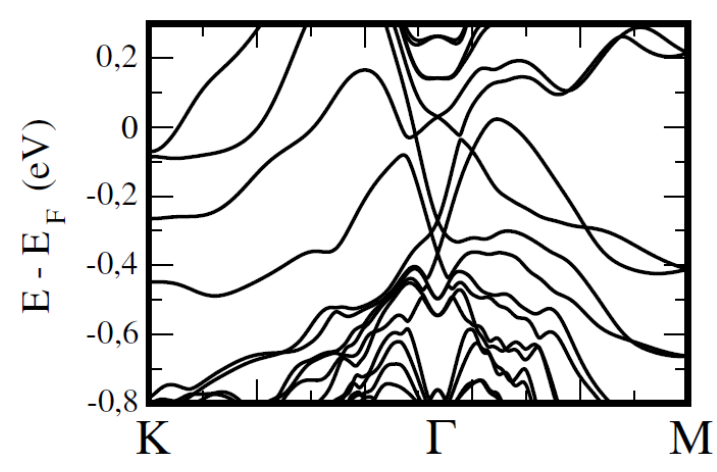

T2

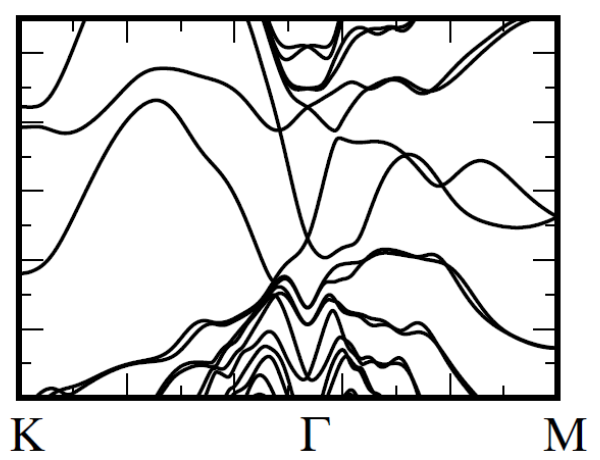

T3

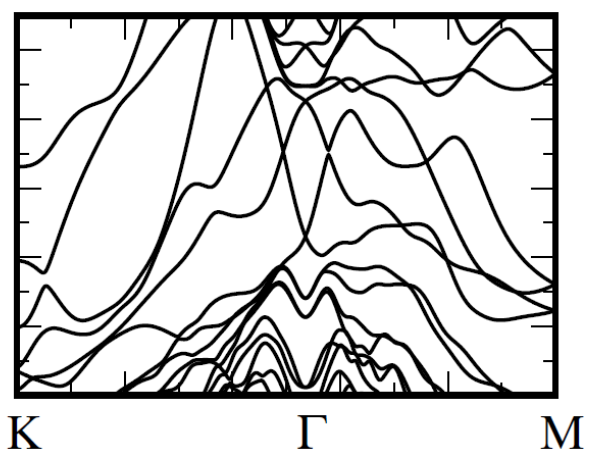

Figura 4.3: Estrutura eletrônica do sistemas $\mathrm{Sn} / \mathrm{Bi}_{2} \mathrm{Se}_{3}$ dado um recobrimento de 100\%, para os sítios a) T1, b) T2, c) T3 e d) T3in.

(superior). A estrutura atômica do sistema T3in é apresentada na imagem (4.4a), a caixa vermelha (verde) encerra os átomos levados em conta na projeção dos estados em vermelho (verde) na (4.4b). Isto revela que, a superfície inferior (na caixa verde) continua contribuindo na dispersão que forma um dos cones de Dirac (o cone 2). Para todos os casos, foi observado que, o cone de Dirac da superfície inferior (o cone 2) permanece em torno do nível de energia $3.0 \mathrm{eV}$, dando um segundo indício de que o estado topológico se mantém. Entretanto, o cone 1 do caso T3in esta formado majoritariamente pelos orbitais dos átomos Se1 e Sn como observado na projeção em vermelho. Foi confirmado que, este cone 2, é formado predominantemente por uma hibridização entre os orbitais $s$ dos Se e os $p_{z}$ dos Sn (figura 4.5).

Diferente ao caso pristino, os cones das superfícies superior e inferior não 


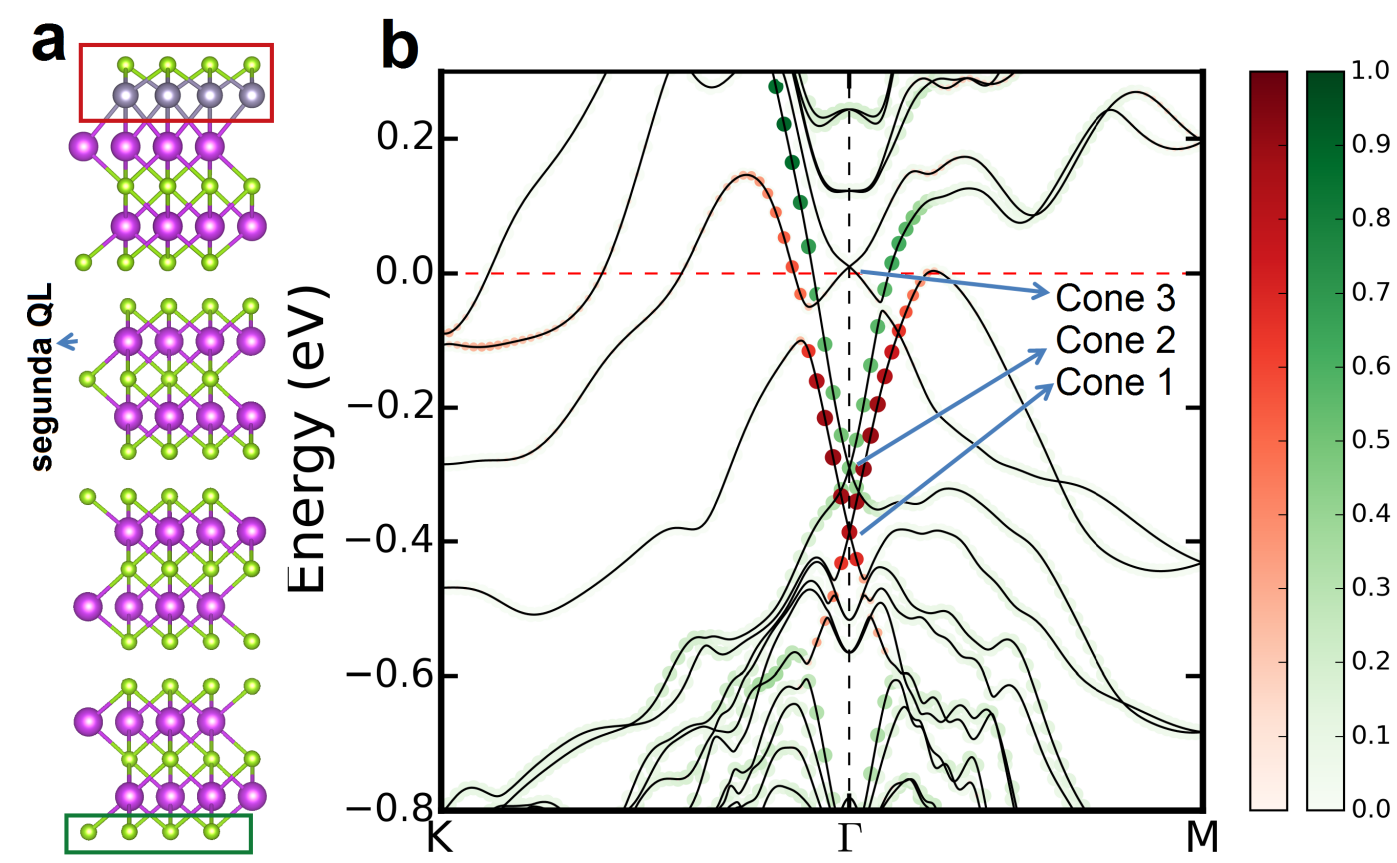

Figura 4.4: Sistema $S n / B i_{2} S e_{3}$ com deposição de Sn no sítio T3in com $100 \%$ de recobrimento; a) Estrutura cristalina após relaxação. O quadro vermelho (verde) encerra os átomos levados em conta na projeção dos orbitais mostrados em na dispersão de energia; b) Estrutura eletrônica com projeção nos orbitais dos átomos das superfícies. Os círculos com cores representam a contribuição dos orbitais dos átomos encerrados nas caixas na estrutura atômica com a cor respectiva.

são bidegenerados, isto é esperado devido à memória do bulk refletida na superfície, porém, a deposição das impurezas quebra a simetria de paridade e com isso a degenerescência dos cones é levantada. Esta quebra de simetria é observada também no formato de Rashba nas bandas localizadas acima do nível de energia de $0 \mathrm{eV}$.

Foi calculada a textura de spin in-plane entorno do $\Gamma$ como mostra-se na figura (4.6). É observado que a textura de spin obedece a simetria de reversão temporal, ou seja, para cada vetor $\boldsymbol{k}$ há um spin s e para $-\boldsymbol{k}$, corresponde um spin $-s$. Percebe-se que a helicidade para cada par de cortes do cone é oposta como no caso usual. Entretanto, o cone 3 também apresenta as características de um cone de Dirac, mesmo não estando localizado na superfície.

Para entender a origem deste terceiro cone de Dirac, é usado como base o modelo efetivo proposto por Zhang que foi apresentado na seção (3.2.3). 

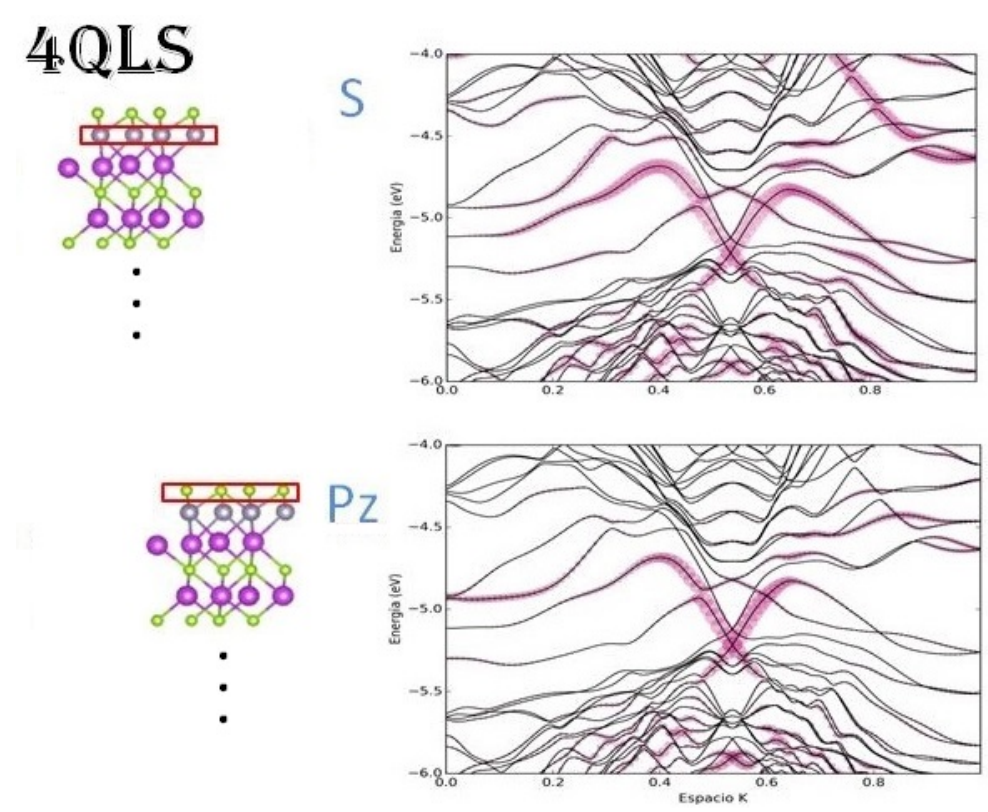

Figura 4.5: Sistema $S n / B i_{2} S e_{3}$ com deposição de Sn no sítio T3in com $100 \%$ de recobrimento. Na estrutura eletrônica é projetada a contribuição dos orbitais $s\left(p_{z}\right)$ dos átomos de Se1 (Sn), é observado que o cone 1 é formado por uma hibridização dos orbitais $s$ dos $\mathrm{Se} 1$ e $p_{z}$ dos Sn.

Pode-se entender as perturbações dos átomos de Sn como uma quebra de simetria de inversão e uma mudança na massa efetiva da segunda QL (sendo a primeira a QL onde é feita a deposição). Como está bem estabelecido, a falta de simetria de inversão resulta em contribuições de ordem maior em $k$ do SOC. O termo de primeira ordem em $k$ é o conhecido termo de Rashba, $h_{R_{1}}(\vec{k})=\alpha_{R_{1}}(\vec{\sigma} \times \vec{k}) \cdot \hat{z}$, este efeito causa um splitting do spin, conforme discutido anteriormente.

De acordo com os resultados DFT, não é observado efeitos de deformação hexagonal no sistema. Assim, consideramos apenas o termo de primeira ordem de Rashba. Portanto, podemos adicionar o termo de Rashba para representar a quebra de simetria de inversão na Hamiltoniana intra-layer do caso pristino, resultando em

$$
\mathcal{H}_{0}=\varepsilon_{0}(\boldsymbol{k})+\left(\begin{array}{cccc}
\mathcal{M}_{0}(\vec{k}) & -i \alpha_{R_{1}} k_{-} & 0 & \bar{A}_{-} \\
i \alpha_{R_{1}} k_{+} & \mathcal{M}_{0}(\vec{k}) & \bar{A}_{+} & 0 \\
0 & \bar{A}_{-} & -\mathcal{M}_{0}(\boldsymbol{k}) & -i \alpha_{R_{1}} k_{-} \\
\bar{A}_{+} & 0 & i \alpha_{R_{1}} k_{+} & -\mathcal{M}_{0}(\boldsymbol{k})
\end{array}\right)
$$




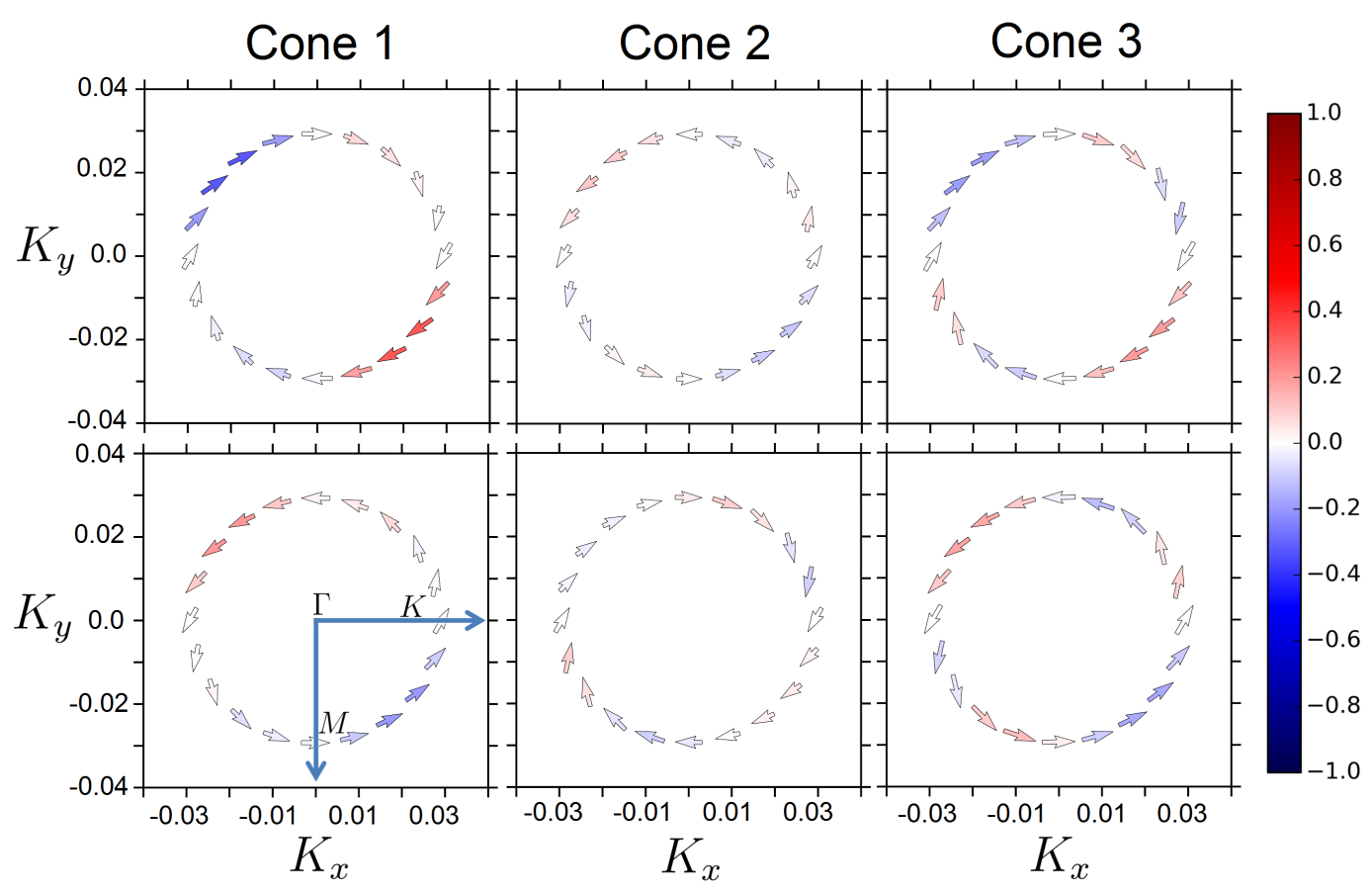

Figura 4.6: Sistema $\mathrm{Sn} / \mathrm{Bi}_{2} \mathrm{Se}_{3}$ com deposição de Sn no sítio T3in com $100 \%$ de recobrimento. Textura de spin helicoidal para diferentes cortes nos cones mostrados na estrutura de bandas. As setas no plano representam as componentes $s_{x}, s_{y}$ do spin, enquanto que a escala de cor representa a componente $s_{z}$. É observado que a helicidade é oposta para cada par de cortes (superior e inferior) sobre o mesmo cone, e a helicidade é correspondente ao sistema protegido por simetria de reversão temporal.

Dado que o termo de Rashba preserva a simetria de reversão temporal, para qualquer valor de $\alpha_{R_{1}}$, isto dá um cone de Dirac para cada superfície. Adicionalmente, a degenerescência do spin é quebrada e o spin-splitting Rashba $E_{R}$ aparece no ponto $\Gamma$ para as bandas do bulk, conforme a figura (4.7a). O valor para o spin-splitting pode ser modificado através do parâmetro de Rashba $\alpha_{R_{1}}$. O spin-spliting Rashba permanece nas bandas do bulk para qualquer valor do parâmetro Rashba maior que zero. Na figura (figura 4.7b), é apresentado o spin-splitting Rashba como função do parâmetro Rashba.

Por outro lado, é esperado que a deposição das impurezas de Sn modifiquem a massa efetiva na segunda QL, por ser a QL próxima aonde ocorre a deposição. Esse efeito pode ser introduzido dentro da energia on-site $\tilde{\varepsilon}(\boldsymbol{k})$, que é 

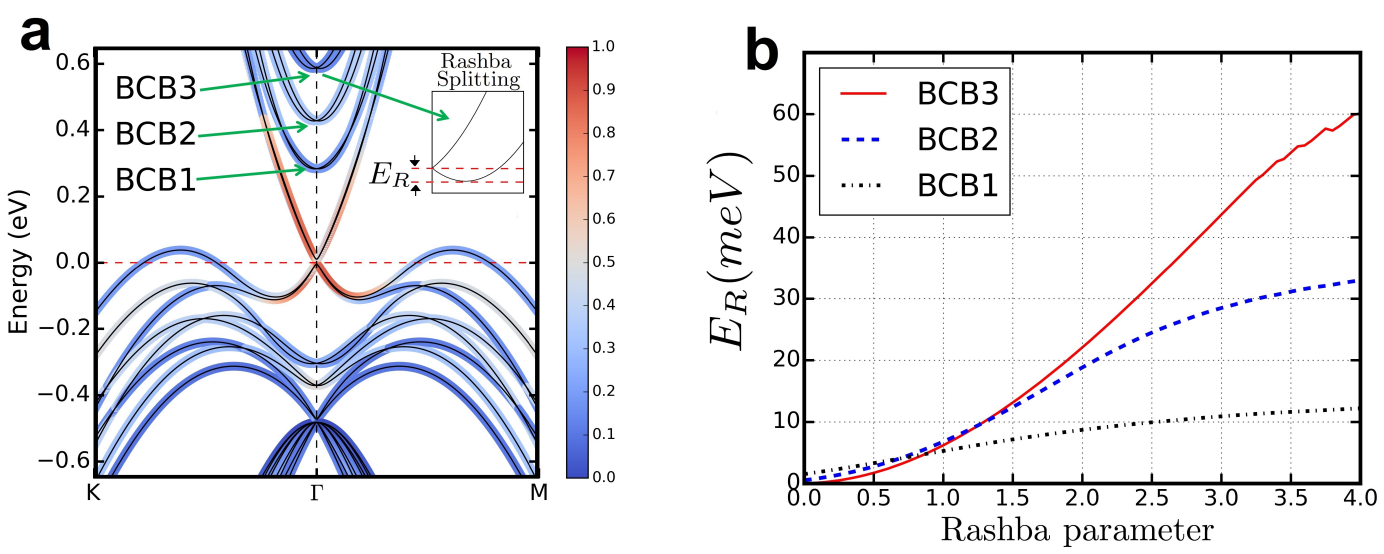

Figura 4.7: Modelo efetivo. (a) Estrutura de bandas da superfície para 4 QLs considerando a quebra de simetria de inversão como um termo de Rashba. Os estados de superfície (bulk) são projetados em vermelho (azul). Nas bandas de condução do bulk (BCB1-3) é observado o splitting Rashba $E_{R}$. (b) Evolução do splitting Rashba em função do parâmetro de Rashba $\alpha_{R_{1}}$ para as bandas de condução do bulk BCB1-3.

redefinida para a segunda QL como

$$
\bar{\varepsilon}(\boldsymbol{k})=\tilde{\varepsilon}(\boldsymbol{k})+\frac{\hbar^{2}}{2 m^{*}} \boldsymbol{k}^{2}+\xi,
$$

onde $m^{*}$ é a massa efetiva, $\xi$ é o shift de energia para o ponto $\Gamma, \tilde{\varepsilon}(\boldsymbol{k})$ é a energia on-site no modelo original de Zhang. Estes termos são incluídos unicamente para a Hamiltoniana intra-layer associada à segunda QL, assim, a dispersão de energia muda conforme o observado na figura (4.8a). Especificamente, o cone de Dirac formado pelos estados de bulk aparece no nível de Fermi (figura 4.8a). Também foi observado que a posição da energia para o ponto $\Gamma$ do cone do bulk induz uma variação na separação $\Delta E$ dos cones da superfície. Esta separação depende da posição de energia do cono do bulk (figura 4.8b). 

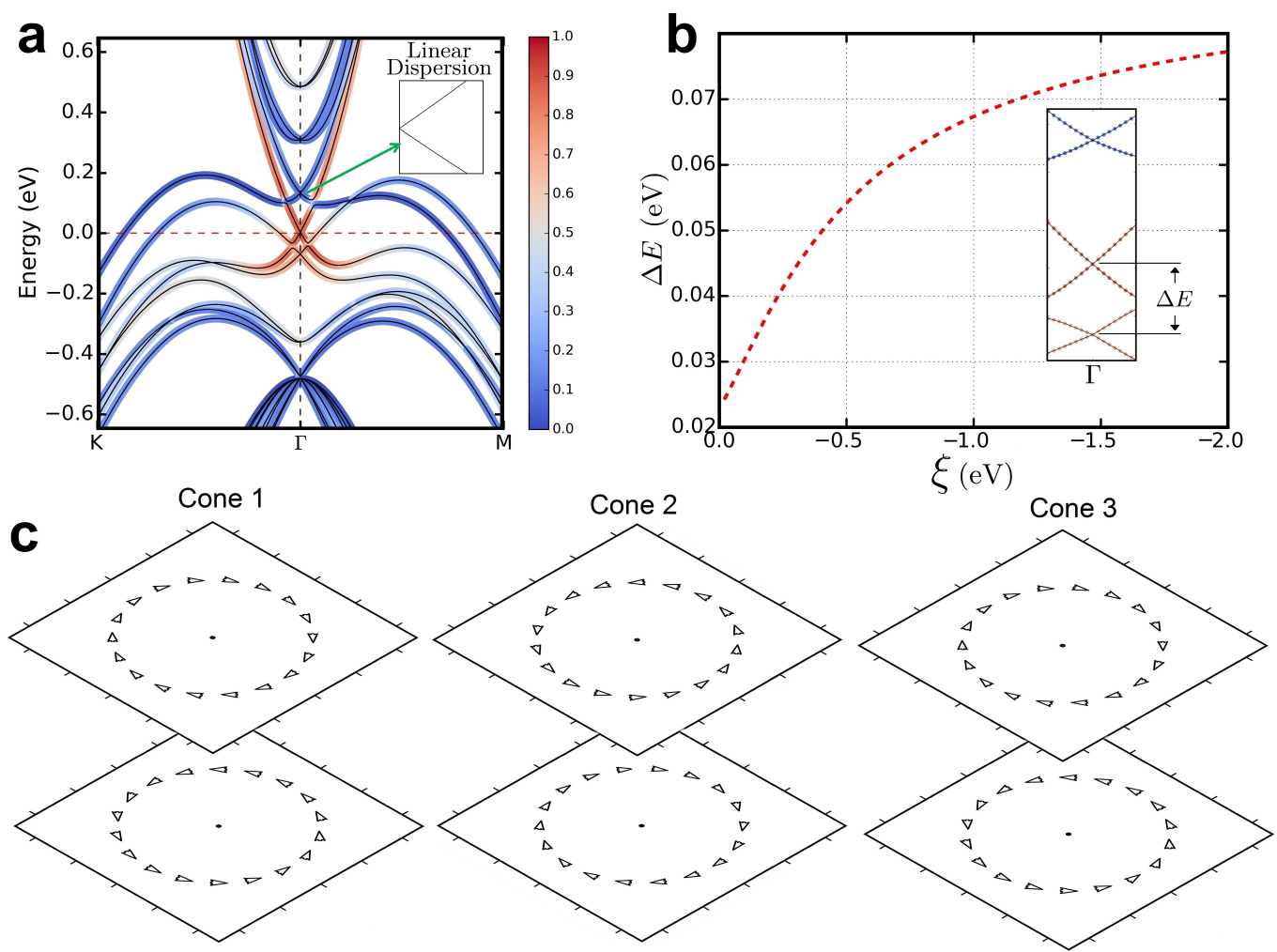

Figura 4.8: a) Efeito da mudança da massa efetiva na dispersão de energia da banda de condução do bulk BCB1. Os estados de superfície (bulk) são projetados em vermelho (azul); b) Relação da separação de energia $\Delta E$ dos cones da superfície com o shift de energia. c) Textura de spin para os cones 1, 2 e 3 do modelo efetivo considerando a quebra de simetria de inversão e modificação da massa efetiva. 


\subsection{Conclusão}

Observamos que quando átomos de Sn são depositados sobre a superfície do $\mathrm{Bi}_{2} \mathrm{Se}_{3}$, pode-se construir um cone de Dirac formado por estados do bulk e localizado entorno do nivel de Fermi. Este cone de Dirac possui textura de spin helicoidal, que sugere quando os estados do bulk estão protegidos pela simetria de reversão temporal, semelhante a dos estados metálicos de superfície. Para todos os sítios de deposição, é preservada a simetria de reversão temporal, de modo que permanecem os estados de superfície topológicos.

Os estados de bulk que formam o cone de Dirac surgem como uma consequência da quebra de simetria de inversão e a variação da massa efetiva na QL próxima à deposição de Sn. Assim, a estrutura de banda e a textura de spin observada nos cálculos DFT foram capturadas no modelo de Zhang ao considerar esses efeitos. 


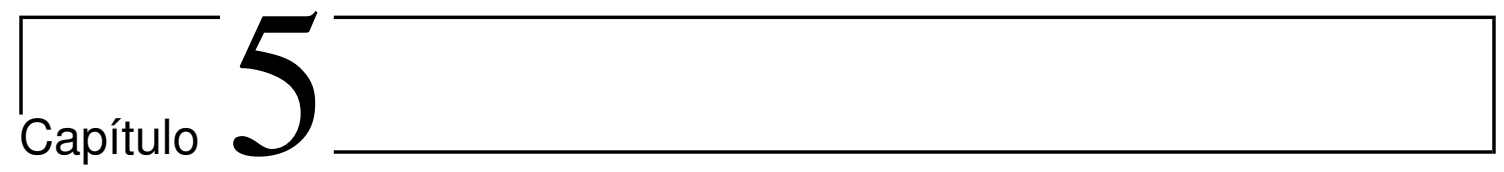

\section{Um Novo Isolante Topológico - 2D}

O principal objetivo da spintrônica é entender os mecanismos pelos quais é possível alcançar o controle eficiente da configuração do spin e das correntes de spin $[2,3]$. Nas últimas décadas, a forma de alcançar este objetivo experimentou um avanço dado a: i) $\mathrm{O}$ descobrimento e entendimento dos mecanismos para gerar correntes de spin em condutores com ordem magnética e em condutores/semicondutores paramagnéticos [65-67]; ii) A proposta teórica dos sistemas injetores de spin que haviam sido propostos experimentalmente [68,69] e iii) A sintetização de materiais 2D com longo tempo de relaxação do spin $[2,10]$.

A geração de correntes de spin, injetores de spin e conservação de spin são mediados pelo acoplamento de spin-órbita (SOC) principalmente via efeito Rashba e/ou efeitos topológicos [34,70-72], tais como o efeito Hall quântico de spin (QSH) [38]. Entretanto, a procura de sistemas que apresentam estas propriedades é uma preocupação principal no desenvolvimento da spintrônica.

Por outro lado, a presença simultânea de um spin-splitting Rashba gigante e efeitos topológicos são reportados apenas em filmes finos e hétero-estruturas de TI $3 \mathrm{D}[73-78]$.

Neste capítulo são estudadas as propriedades topológicas e o efeito Rashba 
em uma família de materiais 2D com estrutura hexagonal, não-centrossimétrico, e formados por átomos dos grupo IV, V e VII. Foi encontrado que o material formado por átomos de $\mathrm{Bi}, \mathrm{Pb}$ e I é um isolante topológico, mecanicamente estável, e tanto no bulk como na nano-fita apresenta estados protegidos pela simetria de reversão temporal. Assim, para colisões elásticas o retroespalhamento é proibido no contorno do nível de Fermi, tanto no bulk como na nano-fita. O sistema PbBiI apresenta um gap de energia $E_{g \Gamma}$ de $0.14 \mathrm{eV}$ e um spin-splitting Rashba $E_{R}$ de $60 \mathrm{meV}$.

A figura (5.1) apresenta a estrutura cristalina obtida por cálculos de primeiros princípios (ab-initio). Os cálculos foram realizados dentro da Teoria Funcional da Densidade (DFT) com a utilização dos códigos SIESTA (Spanish Initiative for Electronic Simulations with Thousands of Atoms) [79] e VASP (Vienna Ab Initio Simulation Package) [80].

Para descrever a energia de troca-correlação foram usadas a aproximação da densidade local [81] (em SIESTA) e a aproximação do gradiente generalizado [82] (em VASP) com o funcional de troca-correlação de Perdew-Burke-Ernzenhof.

\subsection{Propriedades estruturais do PbBiI}

Ao interpretar a rede hexagonal como duas sub-redes triangulares A e B, o sistema tem um átomo da família V sobre a sub-rede A e um dímero IV-VII sobre a sub-rede B. Esta estrutura atômica é não-centrossimétrica, tem um buckled $(d \neq 0)$ e cumpre as operações de simetria do grupo pontual $C_{3 v}: i$ ) Simetria de rotação $R_{3}$ ao longo do eixo $z$; ii) Simetria de espelho $M_{x}(x \rightarrow-x)$ no plano $\left.y z ; i v\right)$ Simetria de reversão temporal $\mathcal{T}$. 


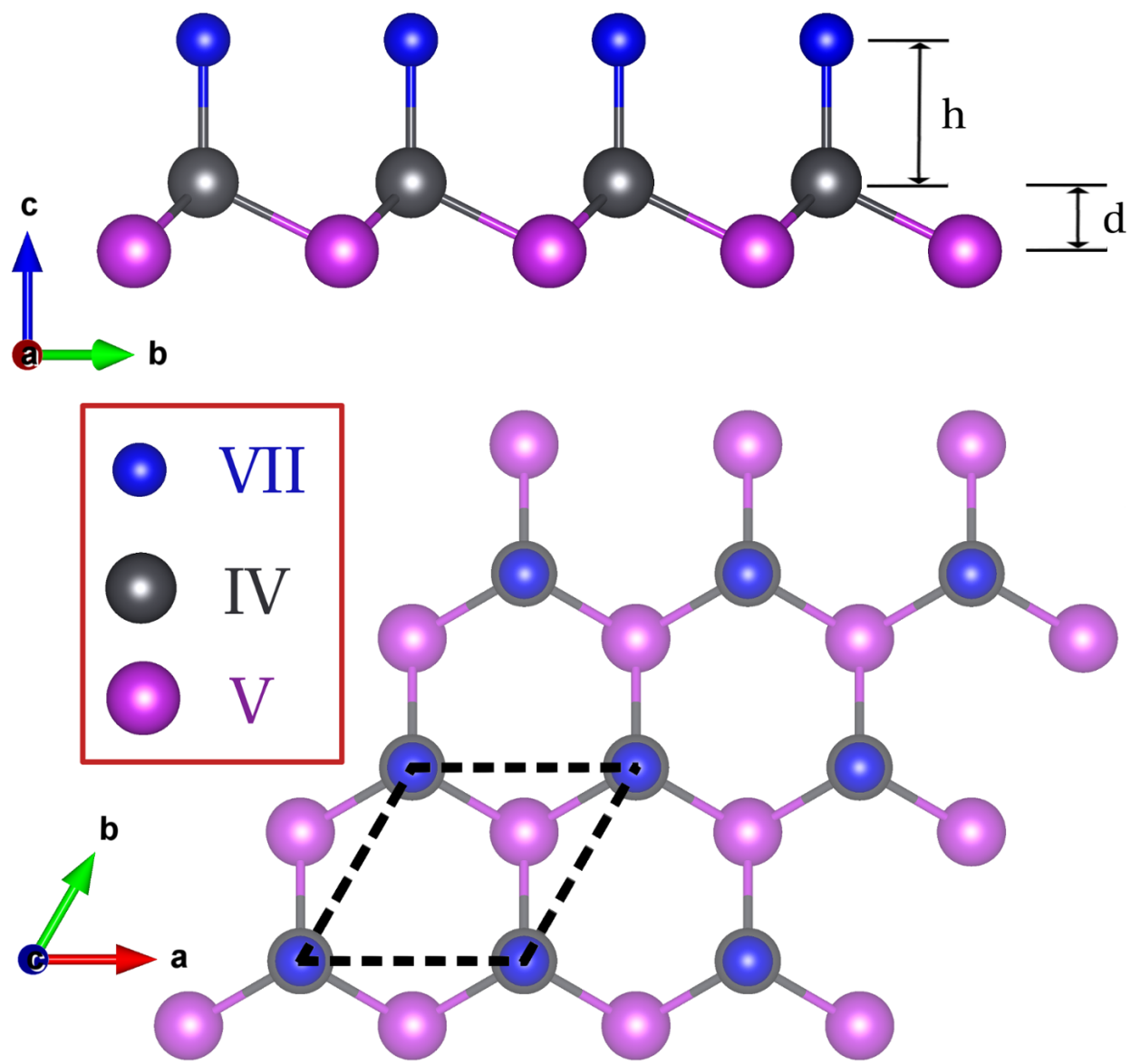

Figura 5.1: a) Vista lateral; b)Superior da estrutura atômica do PbBiI. O $h$ representa o comprimento da ligação do dímero IV-VII, enquanto que o $d$ representa o buckled.

A configuração de mais baixa energia é encontrada por variação do parâmetro da rede (ver apêndice C). No caso específico do sistema $\mathrm{PbBiI}$, o comprimento das ligações $\mathrm{Bi}-\mathrm{Pb}$ e $\mathrm{Pb}-\mathrm{I}(h)$ são $3.04 \AA$ e $1.35 \AA$ respectivamente e um buckled de $1.3 \AA$.

O cálculo ab-initio do espectro de fônons mostra que o sistema formado por átomos de $\mathrm{Bi}, \mathrm{Pb}$, e I é mecanicamente estável (ver apêndice $\mathrm{D}$ ). Por isso, nesta pesquisa focamos nossa atenção sobre as propriedades do sistema $\mathrm{PbBiI}$ cuja energia de formação é $E_{F}=-0.77 \mathrm{eV}$, calculada de acordo com

$$
E_{F}=E_{\mathrm{PbBiI}}-\mu_{\mathrm{Pb}}-\mu_{\mathrm{Bi}}-\mu_{\mathrm{I}},
$$


onde $E_{\mathrm{PbBi}}$ é a energia total do sistema, e $\mu_{\mathrm{Pb}, \mathrm{Bi}, \mathrm{I}}$ é o potencial químico dado na fase mais estável dos respectivos sistemas prístinos $(\mathrm{Pb}, \mathrm{Bi}, \mathrm{I})$.

\subsection{Propriedades eletrônicas do PbBiI}

Em torno do nível de Fermi, a estrutura de bandas possui um caráter dominado majoritariamente pela contribuição dos orbitais $p_{x, y, z}$ dos bismutos. Sem SOC, o topo da banda de valência é dominado pelos orbitais $p_{x, y}$ e o fundo da banda de condução é majoritariamente $p_{z}$ (Figura 5.2a). O sistema não apresenta momento magnético, a simetria de reversão temporal (TR) é preservada e as bandas são degeneradas em spin.
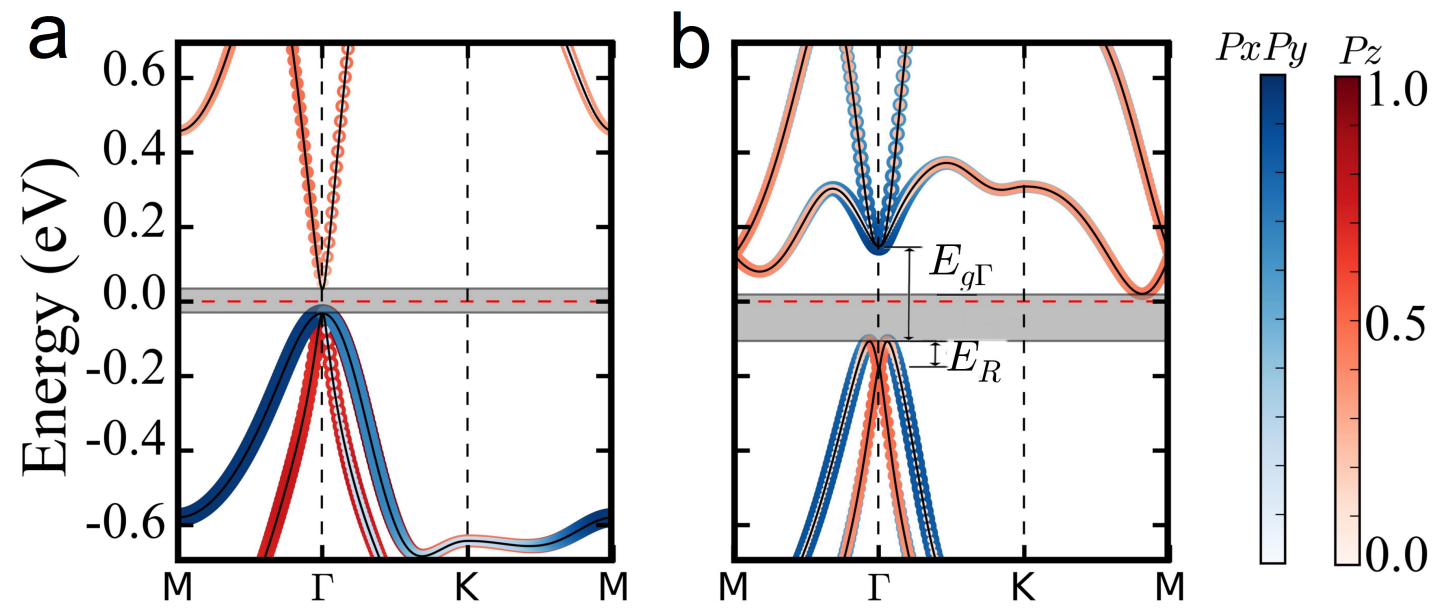

Figura 5.2: Estrutura de bandas a) Sem SOC e b) Com SOC. A escala de cor representa o peso da projeção sobre os orbitais $p_{z}$ e $p_{x}+p_{y}$ do $\mathrm{Bi}$, os quais são indicados por vermelho e azul respectivamente.

Quando o SOC é considerado, os orbitais $p_{x, y, z}$ são misturados e formam orbitais efetivos que preservam o momento angular total. As funções de onda para o ponto $\Gamma$ são formadas pelos estados efetivos $\left\{\left|\Lambda_{J}, j_{z}\right\rangle\right\}$, no qual $J$ é o momento angular total, $j_{z}$ é a projeção do $J$ no eixo $z$, e $\Lambda$ corresponde à contribuição do Bi e/ou Pb-I.

Para preservar o momento angular total, os estados efetivos $\left|\Lambda_{3 / 2}, \pm 3 / 2\right\rangle$ 
devem ser uma combinação linear dos orbitais efetivos $p_{+}=p_{x}+i p_{y}$ e $p_{-}=p_{x}-i p_{y}$, considerando que os estados $\left|\Lambda_{J}, \pm 1 / 2\right\rangle$ são combinações lineares dos orbitais $p$, principalmente orbitais $p_{z}$.

Ao variar a constante de acoplamento do SOC, $\lambda_{\mathrm{SOC}}$, onde $\lambda_{\mathrm{SOC}}=1(0)$ mede o presença (ausência) do SOC, no bulk, foi verificada uma transição de fase topológica devido a uma inversão de banda em $\Gamma$. Esta é caracterizada por um gap em $\Gamma\left(E_{g \Gamma}\right)$, que pode ser igual ao gap geral $\left(E_{g}\right)$, quando é aplicado um strain (ver apêndice C). O sistema passa através de um estado metálico para $\lambda_{\mathrm{SOC}}=0.65$.

No ponto $\Gamma$ a banda de valência e condução são descritos pelos estados efetivos $\left\{\left|\mathrm{Bi}_{J}, j_{z}\right\rangle\right\}$ com $J=3 / 2$ e $J=1 / 2$, respectivamente. Enquanto que, longe do $\Gamma$, os estados efetivos estão misturados.

Fora dos pontos $\Gamma$ e $M$, que são TRIM, a degenerescência no spin é quebrada e manifesta-se uma banda de valência com "forma de m" (M-shape) associada ao efeito Rashba. O spin-splitting Rashba é caracterizado pela energia de splitting, $E_{R}$, como apresentado na figura (5.2b). Conforme o resultado DFT, o $E_{r}$ é de aproximadamente $60 \mathrm{meV}$, que é grande em comparação com os valores encontrados em semicondutores $2 \mathrm{D}$ e é da escala dos maiores encontrados em sistemas 3D [7, 9,73,83-85]. Este fenômeno é robusto a strain, para valores grandes de compressão podem-se obter grandes valores do $E_{R} \approx 90 \mathrm{meV}$ (ver apêndice C).

Com o objetivo de descrever as propriedades do spin do sistema PbBiI, foi comparada a textura de spin out-plane de um material com Rashba usual (Fig 5.3a) e a textura de spin não usual do sistema $\mathrm{PbBiI}($ Fig 5.3b).

Na textura de spin out-plane de materiais com efeito Rashba, tais como nos filmes finos do BiTeI [73], um plano de energia abaixo(acima) do ponto de cruzamento consiste de dois círculos concêntricos com quiralidade do spin in-plane oposta(igual) (Fig 5.3a). Portanto, o retroespalhamento de $\vec{k}$ a $-\vec{k}$ é proibido. 

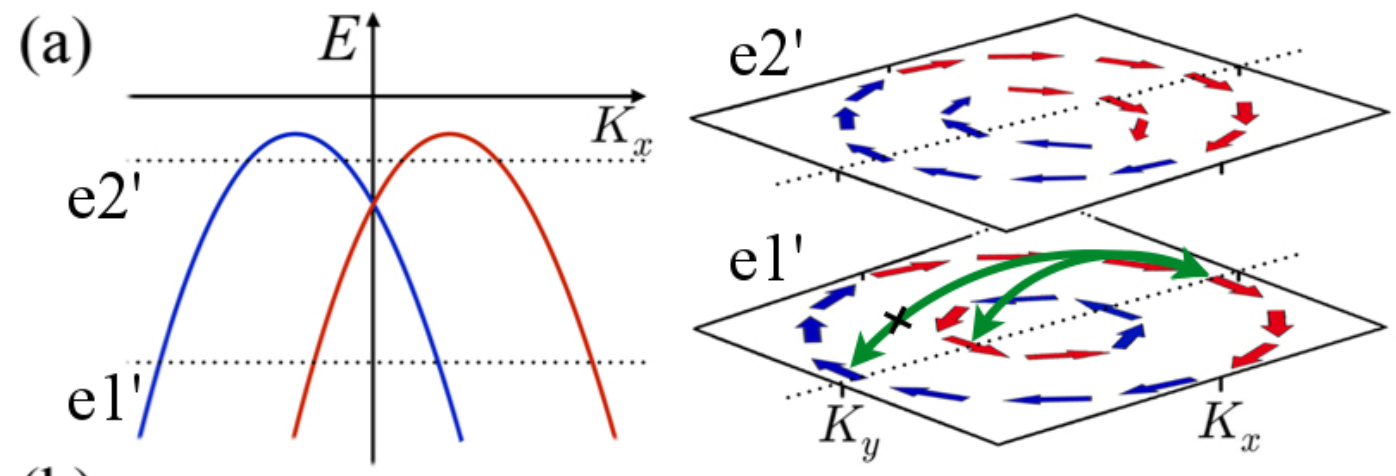

(b)
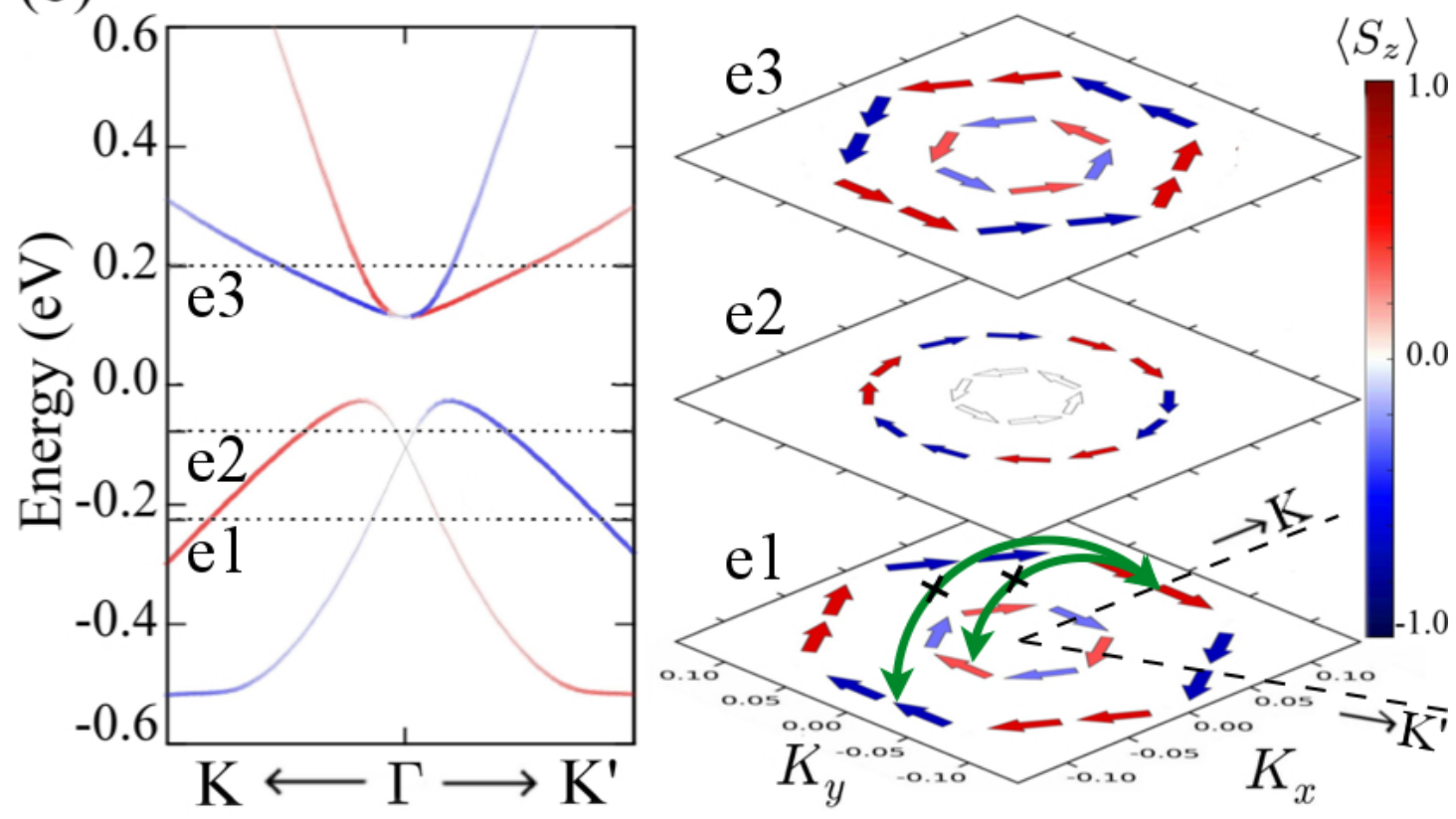

Figura 5.3: Estrutura de bandas e textura de spin out-plane de (a) efeito Rashba usual e (b) o sistema PbBiI. As linhas pontilhadas na estrutura de banda representam os planos de energia (e1-e3 e e1'-e2') nas quais se observa a textura de spin in-plane, cujas grandezas são representadas pelo tamanho das setas. Na estrutura de bandas, as cores azul e vermelho representam a componente $\left\langle S_{z}\right\rangle$ do spin em unidades de $\hbar / 2$. As setas verdes mostram os processos de backscattering permitidos ou proibidos na estrutura de spin, no qual " $\mathrm{x}$ " indica um backscattering proibido. 
Porém, o espalhamento de $\vec{k}$ a $-\vec{k}+\delta k\left(\frac{\vec{k}}{\sqrt{\vec{k} \cdot \vec{k}}}\right)(\operatorname{com} \delta k<\sqrt{\vec{k} \cdot \vec{k}})$ é permitido $[12,86]$, como representado pela seta verde na figura (5.3a).

Entretanto, na banda de valência do sistema PbBiI, um plano de energia abaixo(acima) do ponto de cruzamento consiste de dois círculos concêntricos com a mesma(oposta) quiralidade do spin in-plane, como mostrado nos planos de energia e1 e e2 na figura (5.3b). Na banda de condução, qualquer plano de energia consiste de dois círculos concêntricos com a mesma quiralidade de spin in-plane, como representada nos planos de energia e3 da figura (5.3b).

Adicionalmente, como ocorre nos filmes finos do $\mathrm{Bi}_{2} \mathrm{Te}_{3}$ [87], a polarização de spin out-plane oscila de acordo a simetria $C_{3 v}$. Devido à textura de spin dos estados do bulk, o retroespalhamento de $\vec{k}$ a $-\vec{k}$ é proibido. Porém, contrário ao efeito Rashba usual (Fig 5.3a), as componentes $\left\langle S_{x}\right\rangle$ e $\left\langle S_{y}\right\rangle$ do spin são necessárias para que ocorra espalhamento inelástico de $\vec{k} \mathrm{a}-\vec{k}+\delta k\left(\frac{\vec{k}}{\sqrt{\vec{k} \cdot \vec{k}}}\right)$ (Figura 5.3b).

A proteção contra o retroespalhamento permanece sempre que a simetria TR é preservada. Até onde sabemos, o sistema PbBiI é o primeiro que possui tais propriedades na textura de spin da estrutura de banda no bulk.

Para compreender a origem da inversão de bandas, o spin-splitting Rashba, e a textura de spin não usual obtida via cálculos DFT, foi proposto um modelo simples que captura as propriedades eletrônicas em torno do ponto $\Gamma$.

De acordo com o efeito do SOC discutido anteriormente, foi construída a Hamiltoniana, para descrever o sistema PbBiI, usando a base $\left\{\left|\mathrm{Bi}_{1 / 2}, 1 / 2\right\rangle,\left|\mathrm{Bi}_{1 / 2},-1 / 2\right\rangle,\left|\mathrm{Bi}_{3 / 2}, 3 / 2\right\rangle,\left|\mathrm{Bi}_{3 / 2},-3 / 2\right\rangle\right\}$,

$$
\mathcal{H}=\left(\begin{array}{cc}
\mathcal{H}_{1 / 2}(\vec{k}) & \mathcal{H}_{\text {int }}(\vec{k}) \\
\mathcal{H}_{\text {int }}^{\dagger}(\vec{k}) & \mathcal{H}_{3 / 2}(\vec{k})
\end{array}\right)
$$

Na qual $\mathcal{H}_{1 / 2}(\vec{k})$ e $\mathcal{H}_{3 / 2}(\vec{k})$ são o termos efetivo que descreve os estados $\left|\mathrm{Bi}_{1 / 2}, \pm 1 / 2\right\rangle$ 
e $\left|\mathrm{Bi}_{3 / 2}, \pm 3 / 2\right\rangle$, respectivamente. O termo $\mathcal{H}_{\text {int }}(\vec{k})$ é a interação entre eles.

Os elementos da matriz $\mathcal{H}(\vec{k})$ para o momento angular total $J$ são dados por:

$$
[\mathcal{H}(\vec{k})]_{i j}=\varepsilon_{i j} \delta_{i j}+\sum_{\nu=1}^{6} t_{\vec{a}_{\nu}}^{i j} e^{i \vec{k} \cdot \vec{a}_{\nu}},
$$

onde $i=\left(\mathrm{Bi}, J, j_{z}\right)$ e $j=\left(\mathrm{Bi}, J^{\prime}, j_{z}^{\prime}\right), t_{\vec{a}_{\nu}}^{i j}=\left\langle\vec{n}, \mathrm{Bi}_{J}, j_{z}|H| \vec{a}_{\nu}, \mathrm{Bi}_{J^{\prime}}, j_{z}^{\prime}\right\rangle$ são os termos que descrevem o acoplamento dos vizinhos mais próximos, $\vec{n}$ indica o sítio da rede e $\vec{a}_{\nu}$ corresponde ao $\nu$-ésimo vetor dos seis vizinhos mais próximos. O parâmetro $\varepsilon$ é a energia on-site. Devido aos estados efetivos $\left|(\mathrm{Pb}-\mathrm{I})_{J}, j_{z}\right\rangle$ não ser relevantes em torno do nível de Fermi, podemos omitir os termos de acoplamento associados ao dímero $\mathrm{Pb}-\mathrm{I}$. Ao utilizar as operações de simetria relevantes do grupo pontual $C_{3 v}$, os termos de acoplamento podem ser relacionados uns aos outros para unificar a determinação.

Ao considerar o limite $\vec{k} \rightarrow \Gamma$, é obtida a forma reduzida dos elementos de matriz da hamiltoniana

$$
\mathcal{H}_{1 / 2}(\vec{k})=-\varepsilon^{1 / 2}+h_{0}^{1 / 2}(\vec{k})+h_{R_{3}}^{1 / 2}(\vec{k})+h_{R_{1}}^{1 / 2}(\vec{k})
$$

e

$$
\mathcal{H}_{3 / 2}(\vec{k})=\varepsilon^{3 / 2}-h_{0}^{3 / 2}(\vec{k})+h_{R_{3}}^{3 / 2}(\vec{k})
$$

$\mathrm{Na}$ qual

$$
\begin{gathered}
h_{0}^{J}(\vec{k})=\frac{\hbar^{2}}{2 m_{J}^{*}} \vec{k}^{2}, \\
h_{R_{1}}^{J}(\vec{k})=\alpha_{R_{1}}(\vec{\sigma} \times \vec{k}) \cdot \hat{z} \\
h_{R_{3}}^{J}(\vec{k})=\alpha_{R_{3}}\left[\left(k_{x}+i k_{y}\right)^{3}+\left(k_{x}-i k_{y}\right)^{3}\right] \sigma_{z} \\
\mathcal{H}_{i n t}=\alpha_{i n t} \sigma_{z}\left(k_{x}+i k_{y}\right) .
\end{gathered}
$$



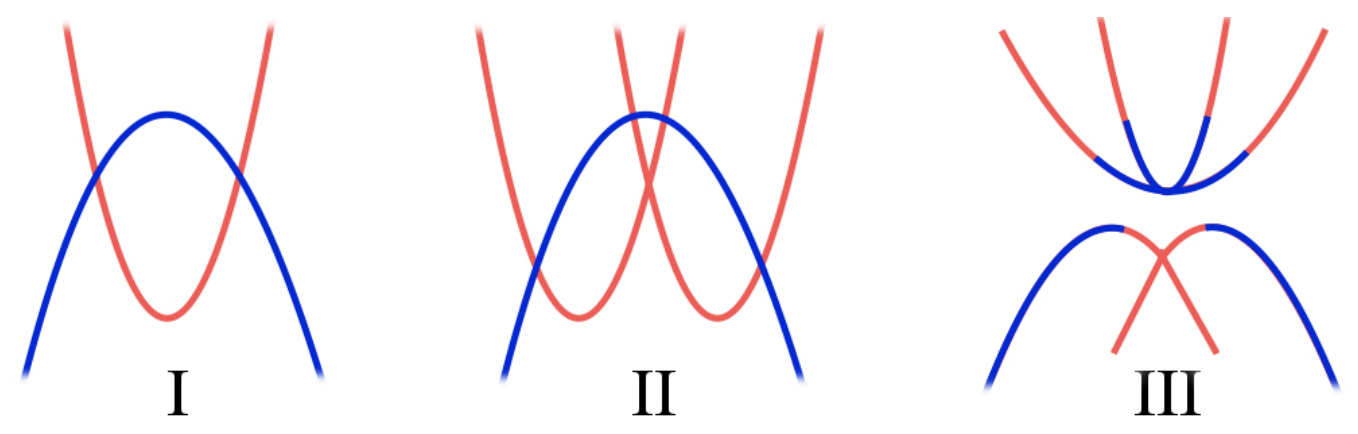

Figura 5.4: Figura esquemática da evolução da estrutura de bandas quando são incluídos os termos de: (I) Energia on-site e dispersão parabólica; (II) Efeito Rashba; (III) Termo de interação entre os estados com diferente momento angular total. A contribuição dos $J=1 / 2(J=3 / 2)$ são representadas pelas linhas vermelhas(azuis).

Estes termos surgem naturalmente como consequência das propriedades de simetria. Os parâmetros $\alpha_{R_{1}}, \alpha_{R_{3}}, \alpha_{\text {int }}$ e $m^{*}$ estão relacionados com os termos de acoplamento. Os parâmetros que ajustam o modelo ao resultado ab-initio estão na (tabela 5.1), os quais são obtidos através de um ajuste por mínimos quadrados.

\begin{tabular}{cccccccc}
\hline \hline$\varepsilon^{1 / 2}(\mathrm{eV})$ & $\varepsilon^{3 / 2}(\mathrm{eV})$ & $m_{1 / 2}^{*}\left(m_{e}\right)$ & $m_{3 / 2}^{*}\left(m_{e}\right)$ & $\alpha_{\text {int }}$ & $\alpha_{R_{1}}$ & $\alpha_{R_{3}}^{1 / 2}$ & $\alpha_{R_{3}}^{3 / 2}$ \\
\hline-0.1764 & 0.1440 & 0.3714 & 0.5294 & $3.5853 i$ & 3.0919 & 12 & 20 \\
\hline
\end{tabular}

Tabela 5.1: Valores dos parâmetros do modelo tigh-binding efetivo para o sistema PbBiI.

A seguir, são analisados separadamente os efeitos de cada termo da Hamiltoniana sobre a estrutura de bandas. O termo $h_{0}^{J}(\vec{k})$ é chamado de aproximação ao elétron quase-livre, que introduz o efeito de banda parabólica. O gap não trivial entre as bandas é de $0.14 \mathrm{eV}$ e é introduzido pelo deslocamento em energia que causa a variação do termo $\varepsilon^{J}$. Uma vez a energia on-site $\varepsilon^{J}$ e a massa efetiva $m_{J}^{*}$ possuam signos opostos, manifesta-se a inversão de banda. Portanto, sem os termos de interação e os termo de Rashba, o modelo da Hamiltoniana leva a um sistema metálico com banda-invertida, que consiste de duas bandas parabólicas como apresentado na figura (5.4-I).

O termo de Rashba $h_{R_{1}}^{J}(\vec{k})$ aparece na componente $\mathcal{H}_{1 / 2}(\vec{k})$, que descreve o topo da banda de valência para o ponto $\Gamma$. Este também representa a interação entre 
o spin, dado pelas matrizes de Pauli $\vec{\sigma}$, e o momento $\vec{k}$. Aqui $\alpha_{R_{1}}$ é a constante de Rashba e $\hat{z}$ o vetor unitário perpendicular plano do sistema. A componente $\mathcal{H}_{3 / 2}(\vec{k})$, descreve o fundo da banda de condução, o qual, não possui o termo $\alpha_{R_{1}}$, portanto, não reproduz o spin-splitting Rashba usual. Ao considerar o feito Rashba é obtida a estrutura de banda apresentada na figura (5.4-II).

A interação entre os estados $\left|B i_{1 / 2}, \pm 1 / 2\right\rangle$ e $\left|B i_{3 / 2}, \pm 3 / 2\right\rangle$ abrem um gap de energia com banda invertida (Fig 5.4-III). Esta interação induz a textura de spin com helicidade in-plane e a polarização do $\left\langle S_{z}\right\rangle$ observada no fundo da banda de condução. Afastado do ponto $\Gamma$, a falta de simetria de inversão estrutural gera termos não lineares no SOC. Portanto, aparecem termos de ordem maior na componente do efeito Rashba [88]. O termo de terceira ordem de Rashba $h_{R_{3}}(\vec{k})$ induz polarização de $\left\langle S_{z}\right\rangle$ e warping hexagonal [87], o qual é observado nas bandas de condução e valência dos resultados DFT, como observado nos cortes de energia e1 e e3 (figura 5.3b). Do nosso conhecimento, não há nenhum outro sistema 2D que experimente este efeito de warping hexagonal nos estados do bulk. De forma análoga aos estados de superfície do Bi2Te3 [87], em trono do nível de Fermi a polarização do $\operatorname{spin}\left\langle S_{z}\right\rangle$ e o warping hexagonal são nulos.

Sem os termos de Rashba de primeira e terceira ordem, o modelo da Hamiltoniana apresentado neste trabalho é equivalente ao modelo BHZ usado para descrever a fase QHS em poços quânticos de HgTe/CdTe [8]. O modelo BHZ considerando o termo de Rashba de primeira ordem $h_{R_{1}}^{J}(\vec{k})$ foi usado para descrever a fase QSH em poços quânticos assimétricos InAs/GaSb/AlSb [89]. As consequências do spin-splitting Rashba gigante e o termo de Rashba de terceira ordem em uma gap com banda invertida, dados como a textura de spin não usual reportados aqui foram ignorados.

Ao realizar os cálculos de estrutura eletrônica na nano-fita do PbBiI, foi verificado que a estrutura de bandas apresenta estados metálicos de borda entre o 

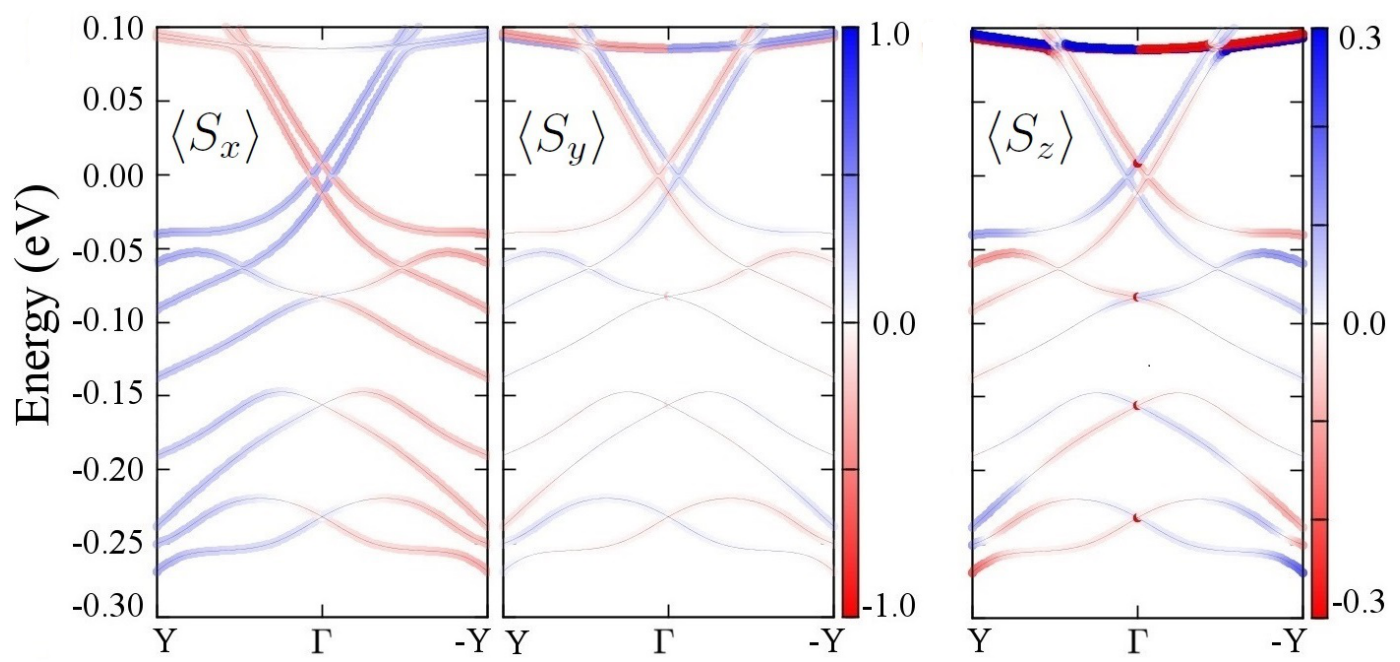

Figura 5.5: Estrutura de banda da nanofita armchair do PbBiI com $98.7 \AA$ de largura. A cor azul (vermelha) representa a projeção no spin up (down) para as componentes $x, y$ e $z$ do spin.

gap de energia presente no bulk. Na figura (5.5) é apresentada a textura de spin da nano-fita armchair. Sobre o ponto $\Gamma$, há dois cones de Dirac formados por bandas com spins opostos.

A simetria de espelho $M_{x}$ é quebrada na nano-fita, portanto, os cones de Dirac localizados nas diferentes bordas são não-degenerados. A textura de spin do bulk discutida anteriormente permanece na estrutura de banda da nano-fita (Fig 5.5). Ou seja, na região de energia mostrada, para qualquer linha constante de energia, os estados $\psi(\vec{k})$ e $\psi(-\vec{k})$ possuem spins opostos. Isto indica que, o retroespalhamento é proibido nos canais de condutividade tanto nos estados do bulk como da superfície. 


\subsection{Conclusão}

Propomos um novo material 2D - O sistema PbBiI - com geometria hexagonal, que apresenta efeito Hall quântico de spin e é mecanicamente estável. Este sistema possui um spin-splitting Rashba de $60 \mathrm{meV}$, gap não trivial de 0.14 eV e um efeito de warping hexagonal. Apresenta spin-splitting Rashba tanto na banda de condução como de valência com a mesma helicidade na textura de spin in-plane. Portanto, sempre que a simetria de reversão temporal é preservada, o retroespalhamento é proibido nos estados do bulk. Para entender as propriedades eletrônicas do sistema PbBiI foi proposto um modelo efetivo de quatro bandas. Neste modelo as bandas de condução e valência são formadas pelos estados efetivos correspondentes ao momento angular total $J=1 / 2$ e $J=3 / 2$, respetivamente. O termo de interação entre estes estados provoca uma transferência de spin do estado $J=1 / 2$ ao estado $J=3 / 2$.

Os estados de borda e do bulk são protegidos perla simetria de reversão temporal, contrário do que ocorre na maioria dos sistemas com fases topológicas não triviais e os estados de bulk não contribuem para o retroespalhamento. A presença simultânea do efeito de Rashba gigante e a topologia de banda não trivial levam à textura de spin não usual resultante, isso abre um caminho para realização de novas aplicações spintrônicas focadas no uso dos estados topológicos de borda. 


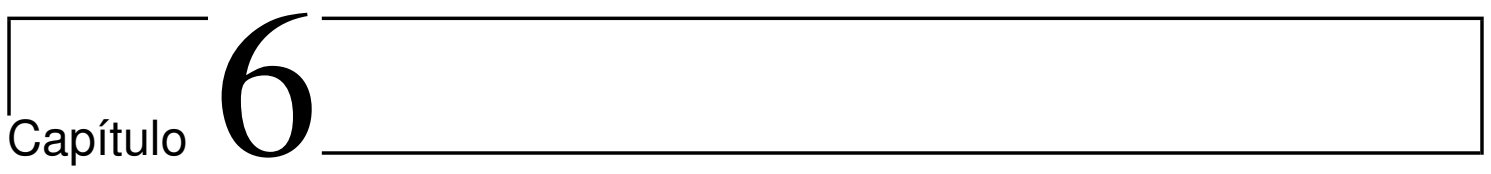

\section{Conclusões gerais}

Nesta dissertação abordamos os conceitos fundamentais da teoria dos isolantes topológicos. A descrição desses materiais foram baseados na teoria do funcional da densidade e no formalismo da equação de Dirac. Mostramos que a partir da assimetria de inversão estrutural surge o efeito Rashba quando o acoplamento spin-órbita é levado em conta. Enfatizamos nas consequências deste efeito na descrição das propriedades eletrônicas dos isolantes topológicos.

Nos resultados obtidos nesta pesquisa foi apresentado o estudo das propriedades estruturais e eletrônicas de dois sistemas. Reportamos o sistema PbBiI como um isolante topológico. O qual apresenta estabilidade mecânica e retroespalhamento proibido tanto nos estados de borda como do bulk, permitindo a geração, conservação e manipulação do spin em uma grande faixa em torno do nível de Fermi. O sistema $\mathrm{Sn} / \mathrm{Bi}_{2} \mathrm{Se}_{3}$ apresenta uma reconstrução na superfície devido a inversão de camadas atômicas. Este é entendido como a manifestação do efeito Rashba devido à quebra de simetria de inversão estrutural. Também foi observado que há um cone de Dirac adicional que pode ser entendido como uma variação de massa efetiva no sistema.

Estes dois sistemas podem ser entendidos com o uso de modelos teóricos 
simples. Estes modelos são propostos a partir das propriedades estruturais e conseguem reproduzir as propriedades eletrônicas destes materiais. 
Parte III

Apêndice 


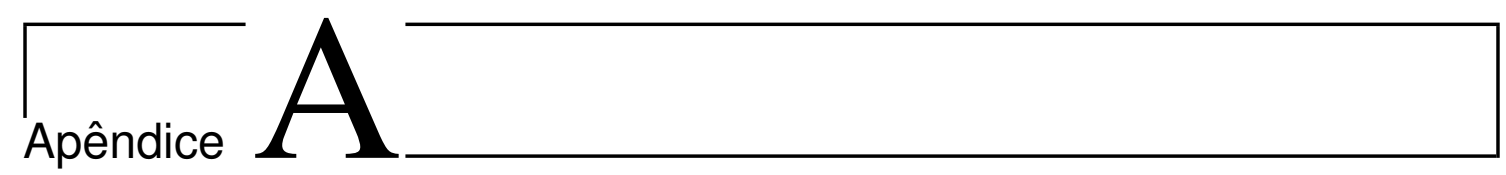

\section{Aproximação de Born Oppenheimer}

Os materiais cristalinos são constituídos por um número grande de núcleos e elétrons que interagem entre eles. Este problema é conhecido como problema de muitos corpos. Na mecânica quântica toda a informação de um sistema está continha numa função de onda $\Psi$ que pode ser encontrada pela solução da hamiltoniana que descreve o sistema. A hamiltoniana de muitos corpos é:

$$
\begin{array}{r}
H=-\sum_{i}^{M} \frac{\hbar^{2}}{2 M_{\vec{R}_{i}}} \nabla_{\vec{R}_{i}}^{2}-\frac{\hbar^{2}}{2 m} \sum_{j}^{N} \nabla_{\vec{r}_{j}}^{2}-\frac{1}{4 \pi \epsilon_{0}} \sum_{i}^{M} \sum_{j}^{N} \frac{Z_{\vec{R}_{i}} e^{2}}{\left|\vec{r}_{j}-\vec{R}_{i}\right|} \\
+\frac{1}{4 \pi \epsilon_{0}} \sum_{j}^{N} \sum_{l<j}^{N} \frac{e^{2}}{\left|\vec{r}_{j}-\vec{r}_{l}\right|}+\frac{1}{4 \pi \epsilon_{0}} \sum_{i}^{M} \sum_{k<i}^{M} \frac{Z_{\vec{R}_{i}} Z_{\vec{R}_{k}} e^{2}}{\left|\vec{R}_{i}-\vec{R}_{k}\right|}
\end{array}
$$

onde $R_{i}, M_{\vec{R}_{i}}$ e $Z_{\vec{R}_{i}}$ são as coordenadas, a massa e a carga do $i$-ésimo núcleo. $\vec{r}_{j}$ e $e$ são as coordenadas e a carga do elétron $j$, e $\epsilon_{0}$ é a permissibilidade do vácuo. É impossível encontrar uma solução exata para esta equação, por isso, é necessário usar métodos alternativos para sua simplificação. Levando em conta a grande diferença que existe entre as massas dos núcleos e os elétrons. Intuitivamente, o primeiro passo na simplificação de esta formulação é a separação dos movimentos nuclear e eletrônico. Então, é possível separar seus movimentos considerando que os núcleos se moverão bem mais lentamente que os elétrons. Esta aproximação é conhecida 
como aproximação de Born Oppenheimer [13]. O resultado dessa aproximação é a separação do problema inicial em dois: um eletrônico e outro nuclear. A função de onda total $\Psi$ do sistema seria um produto das funções de onda eletrônica $\psi$ e nuclear $\phi$, como segue:

$$
\Psi\left(\overrightarrow{r_{i}} ; \overrightarrow{R_{I}}\right)=\psi\left(\overrightarrow{r_{i}} ; \overrightarrow{R_{I}}\right) \phi\left(\overrightarrow{R_{I}}\right)
$$

A função de onda eletrônica $\psi\left(\overrightarrow{r_{i}} ; \overrightarrow{R_{I}}\right)$ depende explicitamente das coordenadas eletrônicas e parametricamente das coordenadas nucleares. Desta forma pode-se simplificar o problema reescrevendo a equação (A.1) como uma parte eletrônica mais um potencial constante que descreve a interação entre íons e elétrons.

$$
H_{\text {elec }}\left(\overrightarrow{R_{I}}\right) \psi\left(\overrightarrow{R_{I}}, \overrightarrow{r_{i}}\right)=\varepsilon_{\text {elec }}\left(\overrightarrow{R_{I}}\right) \psi\left(\overrightarrow{R_{I}}, \overrightarrow{r_{i}}\right)
$$

onde o Halmitoniano eletrônico $H_{\text {elec }}$ é:

$$
H_{\text {elec }}=\sum_{i=1}^{N}-\frac{1}{2} \nabla_{i}^{2}+\sum_{i}^{N} \sum_{I}^{M} \frac{Z_{I}}{\left|\overrightarrow{r_{i}}-\overrightarrow{R_{I}}\right|}+\sum_{i} \sum_{j<i} \frac{1}{\left|\overrightarrow{r_{i}}-\overrightarrow{r_{j}}\right|}
$$

Consequentemente, as funções de onda $\psi$ e as autoenergias $\varepsilon_{\text {elec }}$ dependerão parametricamente das coordenadas nucleares. A energia $\varepsilon_{\text {elec }}$ para um conjunto de pontos $\vec{R}_{I}$ gera uma hipersuperfícies de energia potencial (SEP; surface electrostatic potential). A correspondente SEP obtem-se usando procedimentos de interpolação. E dela a solução da parte nuclear como segue:

$$
\left[\sum_{I} \sum_{J<I} \frac{Z_{I} Z_{J}}{\overrightarrow{R_{I}}-\overrightarrow{R_{J}}}+\varepsilon_{\text {elec }}\left(\overrightarrow{R_{I}}\right)\right] \phi=E \phi
$$

Neste trabalho, o interesse é apenas na parte eletrônica. Mesmo dentro das aproximações, o problema eletrônico não é trivial, e sua solução exata não é conhecida. 


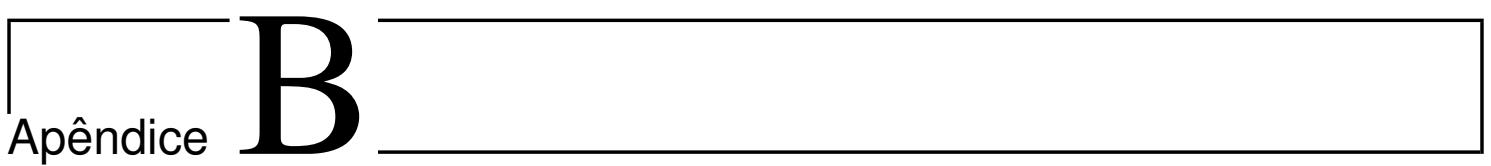

\section{Teoremas de Hohenberg-Kohn}

Teorema 3 O potencial externo $v(r)$ sentido pelos elétrons é um funcional único da densidade eletrônica $\rho(r)$.

Prova. O teorema é provado por redução ao absurdo. Considerando uma Hamiltoniana do tipo:

$$
H=T+U+\sum_{i=1}^{N} v\left(\boldsymbol{r}_{i}\right)
$$

com $\mathrm{T}$ y U os operadores de energia cinética e potencial elétron-elétron respectivamente, e a suma de cada potencial $v(\boldsymbol{r})$ correspondente aos núcleos do sistema o chamado potencial externo $V$. Supõe-se que há dois potenciais externos $V$ e $V^{\prime}$ que proporcionam as distintas hamiltonianas $H$ e $H^{\prime}$ com estados fundamentais $\left|\psi_{0}\right\rangle$ e $\left|\psi_{0}^{\prime}\right\rangle$ e energias $E_{0}$ e $E_{0}^{\prime}$, respectivamente. Se os estados são não degenerados e é usado $\left|\psi_{0}\right\rangle$ como $\left|\psi_{0}\right\rangle$ não é o estado que minimiza $H^{\prime}$ e $\left|\psi_{0}^{\prime}\right\rangle$ não é o estado que minimiza $H$, pode-se escrever

$$
\begin{aligned}
E_{0}^{\prime} & =\left\langle\psi_{0}^{\prime}\left|H^{\prime}\right| \psi_{0}^{\prime}\right\rangle \\
\left\langle\psi_{0}^{\prime}\left|H^{\prime}\right| \psi_{0}^{\prime}\right\rangle & <\left\langle\psi_{0}\left|H^{\prime}\right| \psi_{0}\right\rangle \\
& <\left\langle\psi_{0}|H| \psi_{0}\right\rangle+\left\langle\psi_{0}\left|V^{\prime}-V\right| \psi_{0}\right\rangle \\
& <E_{0}+\int d^{3} r \rho(\boldsymbol{r})\left[v^{\prime}(\boldsymbol{r})-v(\boldsymbol{r})\right]
\end{aligned}
$$




$$
\begin{aligned}
E_{0} & =\left\langle\psi_{0}|H| \psi_{0}\right\rangle \\
\left\langle\psi_{0}|H| \psi_{0}\right\rangle & <\left\langle\psi_{0}^{\prime}|H| \psi_{0}^{\prime}\right\rangle \\
& <\left\langle\psi_{0}^{\prime}\left|H^{\prime}\right| \psi_{0}^{\prime}\right\rangle+\left\langle\psi_{0}\left|V-V^{\prime}\right| \psi_{0}\right\rangle \\
& <E_{0}^{\prime}+\int d^{3} r \rho(\boldsymbol{r})\left[v(\boldsymbol{r})-v^{\prime}(\boldsymbol{r})\right]
\end{aligned}
$$

Somando as relações (B.2) e (B.3), chega-se em

$$
E_{0}+E_{0}^{\prime}<E_{0}+E_{0}^{\prime}
$$

Então, como foi assumido a mesma densidade $\rho(\boldsymbol{r})$ para $v \neq v^{\prime}$, obtêm-se um absurdo decorrente do fato que $\left|\psi_{0}\right\rangle \neq\left|\psi_{0}^{\prime}\right\rangle$. A unicidade de $\rho(\boldsymbol{r})$ exige considerar $\left|\psi_{0}\right\rangle=\left|\psi_{0}^{\prime}\right\rangle$ para o absurdo ser evitado.

Teorema 4 A densidade eletrônica exata de um sistema de muitos elétrons é a que minimiza o funcional de energia total $E[\rho]$.

Prova. O funcional de energia total pode ser escrito como:

$$
E[\rho]=F[\rho]+\langle\psi|V| \psi\rangle
$$

com $F[\rho]=\langle\psi|(T+U)| \psi\rangle$ um funcional universal, ou seja, independe do sistema. Em particular, o estado fundamental é

$$
E\left[\rho_{0}\right]=F\left[\rho_{0}\right]+\left\langle\psi_{0}|V| \psi_{0}\right\rangle
$$

Do princípio variacional de Rayleigh-Ritz, sabe-se

$$
E\left[\psi_{0}\right]<E[\psi]
$$


que também pode ser escrito como

$$
\left\langle\psi_{0}|(T+U)| \psi_{0}\right\rangle+\left\langle\psi_{0}|V| \psi_{0}\right\rangle\langle\langle\psi|(T+U)| \psi\rangle+\langle\psi|V| \psi\rangle \text {. }
$$

Ao utilizar a expressão (B.5), tem-se

$$
E\left[\rho_{0}\right]<E[\rho]
$$

e a densidade de energia do estado fundamental $\rho_{0}$ é a que minimiza o funcional $E[\rho]$. 


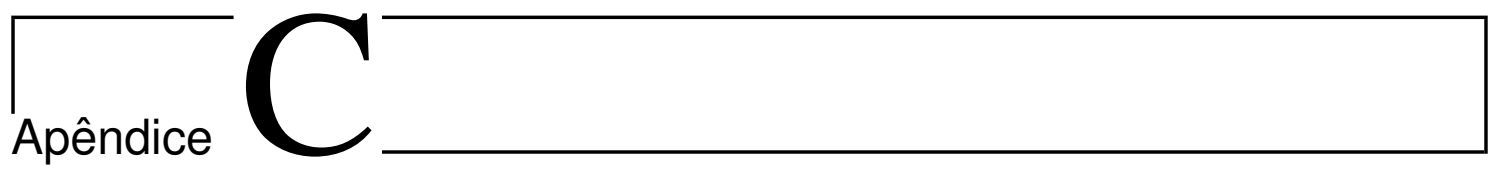

\section{Variação do parâmetro da rede}
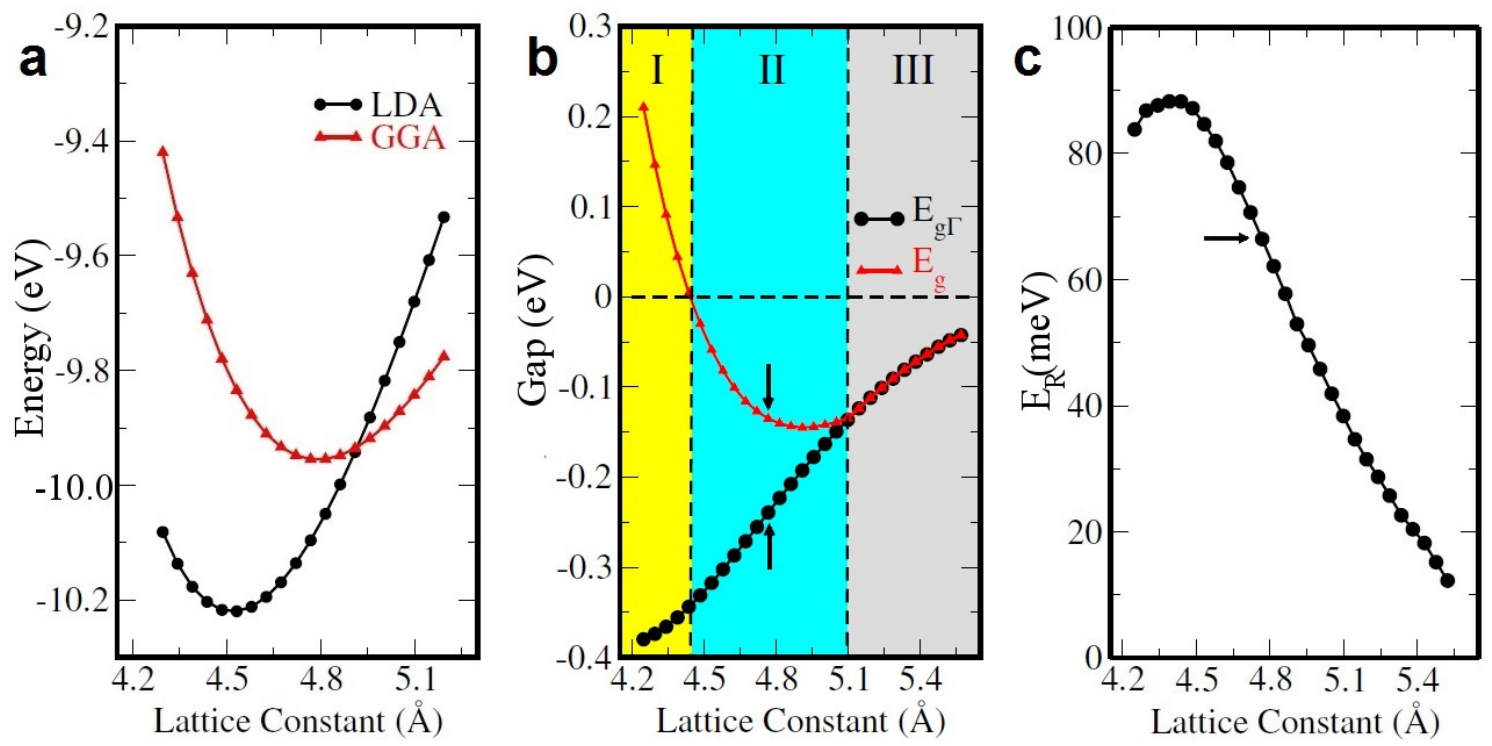

Figura C.1: (a) Energia total do sistema em função da constante de rede para os funcionais de troca-correlação aproximação da densidade local (LDA) e aproximação generalizada do gradiente (GGA). (b) Gap de energia em função da constante de rede, onde $E_{g}$ e $E_{g \Gamma}$ são o gap do sistema e o gap no ponto $\Gamma$, respectivamente (ver figura 5.2 ). Abaixo da linha tracejada a 0.0, $E_{g \Gamma}$ é invertido. (c) spin-splitting Rashba em função da constante de rede. A seta representa a constante de rede para quando o sistema é energeticamente favorável segundo o funcional GGA.

Usando o funcional de troca-correlação segundo a aproximação do gradiente generalizado (GGA), encontramos que a constante de rede $a$ é $4.77 \AA$, enquanto que, com a aproximação da densidade local (LDA) é $4.53 \AA$, conforme a 
Fig (C.1a).

Achamos que substituindo os átomos de $\mathrm{Pb}$ por átomos de $\mathrm{Sn}$ (ou mudando a constante de rede) permite o controle do gap (ver Fig C.1b) e o spinsplitting Rashba (ver Fig C.1c) A diminuição (aumento) da constante de rede pode ser entendido como uma deformação de compressão (elongação) do material. Dependendo da intensidade da deformação, podemos observar diferentes enfoques: Semi-metal, semicondutor de gap indireto e semicondutor de gap direto, como representado na figura (C.1b) para as regiões I, II e III, respectivamente. A posição de equilíbrio é representada pela seta (Fig C.1). Como mencionado anteriormente, na região II, no ponto onde o sistema está em equilibro com o parâmetro da rede, $E_{g \Gamma}$ é negativo indicando uma banda invertida para o ponto $\Gamma$.

Na regão onde é aplicada a deformação de compressão ( a < 4.77), conforme comprimimos o sistema, o fundo da banda de condução, localizado na direção $M \rightarrow K$, decresce e cruza o topo da banda de valência para $a=4.3 \AA$, formando um semi-metal (região I). Na regão onde é aplicada a deformação de elongação ( $\mathrm{a}>4.77 \AA$ ), o fundo da banda de condução, que inicialmente é localizado na direção $M \rightarrow K$, aumenta. Para o ponto (Constante de rede $>5.1 \AA$ ) o $E_{g} \mathrm{e}$ $E_{g \Gamma}$ passam a ser o mesmo, formando um semicondutor de gap direto no ponto $\Gamma$ (região III).

Para qualquer valor da deformação considerada aqui, o carácter das bandas entorno do ponto $\Gamma$ permanecem invertidas. Assim, dado que a inversão de banda é associada com o estado topológico, podemos dizer que a topologia não trivial do sistema é robusto a estas deformações, sempre que a deformação não seja maior que aquelas consideradas aqui, como mostrado na figura C.1. 


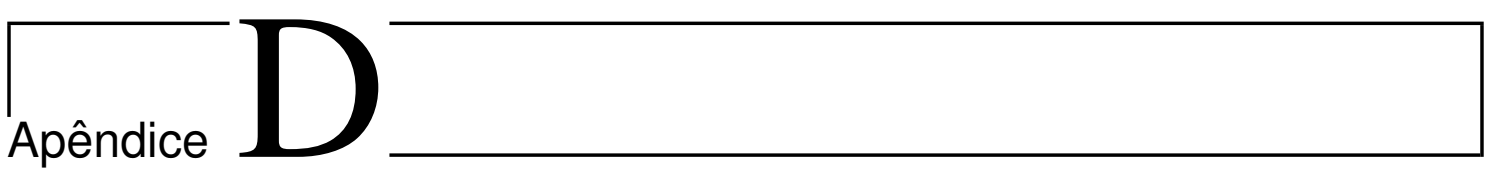

\section{Estabilidade mecânica do PbBiI}

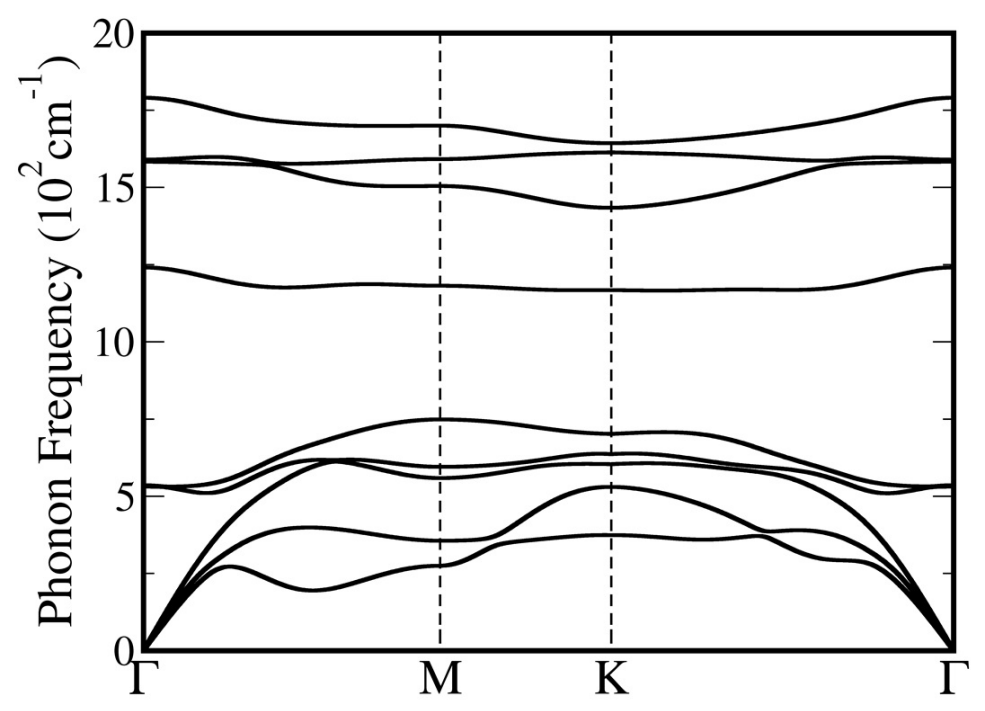

Figura D.1: Dispersão de fônons para o sistema PbBiI calculado por primeiros princípios.

Conforme o observado na figura (D.1), no existem frequências negativas de vibração para o sistema PbBiI. A energia de formação é de $-0.77 \mathrm{eV}$, calculada acorde a $E_{F}=E_{\mathrm{PbBiI}}-\mu_{\mathrm{Pb}}-\mu_{\mathrm{Bi}}-\mu_{\mathrm{I}}$, onde $E_{\mathrm{PbBiI}}$ é a energia do sistema PbBiI e $\mu_{\mathrm{Pb}, \mathrm{Bi}, \mathrm{I}}$ é o potencial químico da fase mais estável dos respetivos bulk $\mathrm{Pb}, \mathrm{Bi}$ e I. Estas propriedades são necessárias e suficientes para concluir que o a estrutura PbBiI apresentado aqui é mecanicamente estável. 


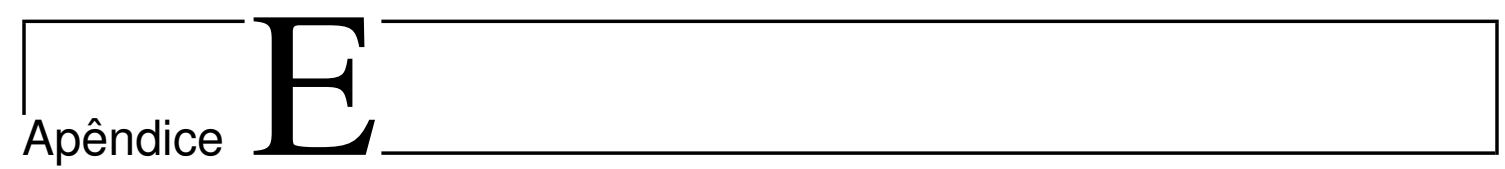

Artigo publicado 


\title{
Unconventional spin texture in a noncentrosymmetric quantum spin Hall insulator
}

\author{
C. Mera Acosta, ${ }^{1, *}$ O. Babilonia, ${ }^{1}$ L. Abdalla, ${ }^{2}$ and A. Fazzio ${ }^{1,3}$ \\ ${ }^{1}$ Instituto de Física, Universidade de São Paulo, CP 66318, 05315-970, São Paulo, SP, Brazil \\ ${ }^{2}$ University of Colorado, Boulder, Colorado 80309, USA \\ ${ }^{3}$ Centro de Ciências Naturais e Humanas, Universidade Federal do ABC, Santo André, CP 09210-170, São Paulo, Brazil
}

(Received 12 January 2016; revised manuscript received 2 May 2016; published 15 July 2016)

\begin{abstract}
We propose that the simultaneous presence of both Rashba and band inversion can lead to a Rashba-like spin splitting formed by two bands with the same in-plane helical spin texture. Because of this unconventional spin texture, the backscattering is forbidden in edge and bulk conductivity channels. We propose a noncentrosymmetric honeycomb-lattice quantum spin Hall (QSH) insulator family formed by the IV, V, and VII elements with this property. The system formed by $\mathrm{Bi}, \mathrm{Pb}$, and $\mathrm{I}$ atoms is mechanically stable and has both a large Rashba spin splitting of $60 \mathrm{meV}$ and a large nontrivial band gap of $0.14 \mathrm{eV}$. Since the edge and the bulk states are protected by the time-reversal (TR) symmetry, contrary to what happens in most doped QSH insulators, the bulk states do not contribute to the backscattering in the electronic transport, allowing the construction of a spintronic device with less energy loss.
\end{abstract}

DOI: 10.1103/PhysRevB.94.041302

The main objective of spintronics is to understand the mechanisms by which it is possible to achieve efficient control of both spin configurations and spin currents $[1,2]$. In the past decade, the way to achieve this objective has experienced a breakthrough due to (i) the discovery and understanding of mechanisms to generate spin currents in conductors with magnetic order and in paramagnetic conductors and semiconductors [3-5], (ii) the experimental observation of theoretically proposed spin injector systems [6-8], and (iii) the synthesis of two-dimensional (2D) materials with long spin relaxation time [1,9]. The generation of spin currents, spin injections, and spin conservation are mediated by the spin-orbit coupling (SOC), mainly via Rashba effect and/or nontrivial topological phases [10-13], such as the quantum spin Hall (QSH) effect [14]. Therefore, the search for systems experiencing these properties is a primary concern for the development of spintronics.

QSH insulators support helical metallic edge states, forming topological Dirac fermions protected by the time-reversal (TR) symmetry on an insulating bulk [6,7]. The topological transition from trivial insulating to topological insulators is evidenced as a band inversion at the TR invariant $k$-point mediated by the SOC. The topological band dispersion has been experimentally characterized via angle-resolved photoemission spectroscopy (ARPES) and local scanning tunneling microscopy (STM) in three-dimensional (3D) topological insulators [6] and via transport measurements in $\mathrm{HgTe} / \mathrm{CdTe}$ quantum wells $[15,16]$. On the other hand, the Rashba effect, arising from the lack of inversion symmetry, leads to parallel spin-polarized band dispersion curves with opposite in-plane chiral spin texture [17], allowing the control of the spin direction through an electric field $[10,12,13]$. These dispersion curves and Fermi contours have been characterized by spectroscopic measurements for many surfaces and interfaces [18-21]. Large Rashba spin splitting are found in materials formed by heavy elements with strong intrinsic SOC such as $\mathrm{Bi}, \mathrm{Pb}$, and $\mathrm{W}$, among others [21-25]. In this work,

\footnotetext{
*acosta@if.usp.br
}

we look at the consequences of the simultaneous presence of a Rashba spin splitting and a inverted band gap. Such properties appear simultaneously in thin films and heterostructures of 3D topological insulators [26-31].

Here, we show that bulk states can be protected against backscattering in nanoribbons of QSH insulators with bulk inversion asymmetry. This behavior is a consequence of the simultaneous presence of both Rashba and band inversion in a QSH insulator. In our model, both the conduction and the valence bands are formed by two bands with the same in-plane helical spin texture and opposite $\left\langle S_{z}\right\rangle$ spin component. We propose a stable, noncentrosymmetric honeycomb-lattice QSH insulator that presents this unconventional bulk spin texture. This system is formed by the $\mathrm{Bi}, \mathrm{Pb}$, and I elements and has a large nontrivial band gap of $0.14 \mathrm{eV}$ and a huge Rashba spin splitting of $60 \mathrm{meV}$. To construct the Hamiltonian exhibiting the proposed spin texture, we will use the PbBiI system.

Figure 1 summarizes the crystalline structure and the results we obtain from $a b$ initio calculations, which are performed within the density functional theory (DFT) framework as implemented in the SIESTA code [32] and in the VIENNA AB INITIO SIMULATION PACKAGE (VASP) [33]. We consider the on-site approximation for the SOC [34,35] in the SIESTA code. The local density approximation [36] and the PerdewBurke-Ernzenhof generalized gradient approximation [37] are used for the exchange-correlation functional. Interpreting the hexagonal lattice as two triangular sublattices $\mathrm{A}$ and $\mathrm{B}$, the system has a $\mathrm{V}$ atom type on the sublattice $\mathrm{A}$ and a IV-VII dimer in the sublattice B [Fig. 1(a)]. These noncentrosymmetric systems have a buckled format and fulfill the symmetry operations of the $C_{3 v}$ symmetry: (i) threefold rotation symmetry $R_{3}$ along the $z$ axis, (ii) mirror symmetry $M_{x}(x \rightarrow-x)$ in the $y z$ plane, and (iii) TR symmetry $\mathcal{T}$. We predict that the $\mathrm{PbBiI}$ system is mechanically stable, imaginary frequencies does not exist in the ab initio phonon spectrum (see the Supplemental Material [38]), and the formation energy $\left(E_{F}=E_{\mathrm{PbBiI}}-\mu_{\mathrm{Pb}}-\mu_{\mathrm{Bi}}-\mu_{\mathrm{I}}\right)$ is about $-0.77 \mathrm{eV}$.

At the $\Gamma$ point, the top of the valence band is dominated by the $p_{x, y} \mathrm{Bi}$ orbitals and the bottom of the conduction band mainly consists of the $p_{z} \mathrm{Bi}$ orbitals, as shown in Fig. 1(b). 

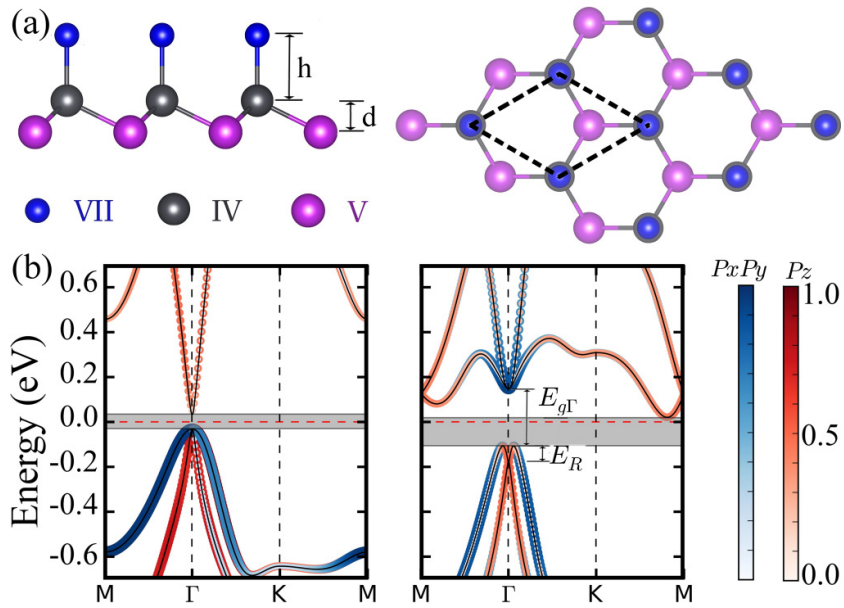

FIG. 1. (a) Top and side views of the PbBiI atomic structure. In the lowest energy configuration the buckled, $d$, is $1.3 \AA$ in length and, the $\mathrm{Bi}-\mathrm{Pb}$ and $\mathrm{Pb}-\mathrm{I}(h)$ bounds are $3.04 \AA$ and $1.35 \AA$ in length, respectively. (b) Band structure without SOC (left) and with SOC (right). The color scales represent the weight of the orbital projection in the wave function $\psi_{n}(\vec{k})$. The projections in the $p_{z}\left(p_{x}\right.$ and $\left.p_{y}\right) \mathrm{Bi}$ orbitals are indicated by red (blue). The Rashba spin splitting and the band inversion are characterized by $E_{R}$ and $E_{g \Gamma}$, respectively.

When the SOC is taken into account, the $p$ orbitals are mixed to form effective orbitals preserving the total angular momentum and a band inversion occurs when $\lambda_{\text {SOC }}=0.65$, where $\lambda_{\text {SOC }}=0(1)$ means the absence (full presence) of SOC. We implemented the evolution of Wannier center of charges as an alternative method to the $Z_{2}$ invariant calculation using $a b$ inito simulations [39-41]. We find that there is no horizontal reference line that crosses the evolution of the WCCs at least an odd number of times (see the Supplemental Material), showing a value of $Z_{2}=1[39,41]$, and hence confirming that the PbBiI system is a QSH insulator. On the other hand, according to the symmetry operation, the wave function at the $\Gamma$ point is given by the $\left\{\left|\Lambda_{J}, j_{z}\right\rangle\right\}$ effective states, where $J$ is the total angular momentum, $j_{z}$ is the projection along the $z$ axes, and $\Lambda$ corresponds to the $\mathrm{Bi}$ and $\mathrm{Pb}-\mathrm{I}$ contributions. To preserve the total angular momentum, the $\left|\Lambda_{3 / 2}, \pm 3 / 2\right\rangle$ effective states should be a linear combination of the $p_{+}=p_{x}+i p_{y}$ and $p_{-}=p_{x}-i p_{y}$ effective orbitals and the $\left|\Lambda_{J}, \pm 1 / 2\right\rangle$ effective states should be a linear combination of the $p$ orbitals, mainly $p_{z}$ orbitals. In this inverted band gap the conduction band mainly consists of $p_{x, y} \mathrm{Bi}$ orbitals and the valence band is formed by the $p$ orbitals, mainly dominated by $p_{z}$ Bi orbitals, as shown in Fig. 1(b). Therefore, at the $\Gamma$ point, the valence (conduction) band is described by the effective states $\left\{\left|\mathrm{Bi}_{J}, j_{z}\right\rangle\right\}$ with $J=3 / 2(J=1 / 2)$ and hence, we write the Hamiltonian using the full SOC basis $\left\{\left|\mathrm{Bi}_{1 / 2}, 1 / 2\right\rangle,\left|\mathrm{Bi}_{1 / 2},-1 / 2\right\rangle,\left|\mathrm{Bi}_{3 / 2}, 1 / 2\right\rangle,\left|\mathrm{Bi}_{3 / 2},-1 / 2\right\rangle\right\}$. by

The tight-binding Hamiltonian matrix elements are given

$$
[\mathcal{H}(\vec{k})]_{i j}=\varepsilon_{i j} \delta_{i j}+\sum_{\nu=1}^{6} t_{\vec{a}_{\nu}}^{i j} e^{i \vec{k} \cdot \vec{a}_{\nu}},
$$

where $i=\left(\mathrm{Bi}, J, j_{z}\right), j=\left(\mathrm{Bi}, J^{\prime}, j_{z}^{\prime}\right)$ and $\varepsilon$ is the onsite energy. Since the $\left|(\mathrm{Pb}-\mathrm{I})_{J}, j_{z}\right\rangle$ effective states' contribution is not relevant near the Fermi energy, we omit the terms associated with the nearest neighbors (Pb-I dimer) and hence $t_{\vec{a}_{v}}^{i j}=$ $\left\langle\vec{n}, \mathrm{Bi}_{J}, j_{z}|H| \vec{a}_{\nu}, \mathrm{Bi}_{J^{\prime}}, j_{z}^{\prime}\right\rangle$ represents the next nearest neighbor hopping terms, with $\vec{n}$ indicating the lattice site and $\vec{a}_{v}$ corresponding to the $v$ th of the six next nearest neighbor vectors. Different from buckled honeycomb-lattice systems, such as germanene, and silicene among others, in which the nearest neighbor hopping terms are essential to their descriptions, in the $\mathrm{PbBiI}$ the $\mathrm{Pb}-\mathrm{I}$ dimers effect is introduced within the $\mathrm{Bi}$ atoms hopping term. Therefore, the $\mathrm{PbBiI}$ Hamiltonian is strikingly different from the Kane-Mele model [14]. Using the relevant symmetry operations of the $C_{3 v}$ point group, these hopping terms can be related to each other and are uniquely determined (see the Supplemental Material), which leads to an approximate description of the DFT band structure [see Fig. 2(a)].

Considering the $\vec{k} \rightarrow \Gamma$ limit we obtain a reduced form for the tight-binding Hamiltonian matrix elements,

$$
\mathcal{H}_{J}(\vec{k})=(-1)^{2 J+1} \varepsilon_{J}+(-1)^{2 J} h_{J, 0}(\vec{k})+h_{J, R_{3}}(\vec{k})+h_{J, R_{1}}(\vec{k}),
$$

where $h_{0}^{J}(\vec{k})=\xi \vec{k}^{2}, h_{R_{3}}^{J}(\vec{k})=\alpha_{R_{3}}^{J}\left[\left(k_{+}\right)^{3}+\left(k_{-}\right)^{3}\right] \sigma_{z}, h_{R_{1}}^{J}(\vec{k})=$ $\alpha_{R_{1}}^{J}(\vec{\sigma} \times \vec{k}) \cdot \hat{z}$, and $\mathcal{H}_{\text {int }}=\gamma \vec{\sigma} \cdot \vec{k}$. Here, $\mathcal{H}_{1 / 2}(\vec{k})$ and $\mathcal{H}_{3 / 2}(\vec{k})$ are the effective terms that described the $\left|\mathrm{Bi}_{1 / 2}, \pm 1 / 2\right\rangle$ and $\left|\mathrm{Bi}_{3 / 2}, \pm 3 / 2\right\rangle$ states, respectively, and $\mathcal{H}_{\text {int }}(\vec{k})$ is the interaction between these states. The parameters are related to the hopping terms and are obtained via a least squares standard approach in order to match the DFT calculation (see the Supplemental Material). Since we find that $\alpha_{R_{1}}^{3 / 2}(\vec{k}) \approx 0$ and considering $\xi^{1 / 2} \approx \xi^{3 / 2}=\xi$, we rewrite the Hamiltonian as

$$
\mathcal{H}(\vec{k})=\left(\begin{array}{cccc}
-\varepsilon+\xi k^{2} & i \alpha_{R_{1}} k_{-} & 0 & \gamma k_{-} \\
-i \alpha_{R_{1}} k_{+} & -\varepsilon+\xi k^{2} & \gamma k_{+} & 0 \\
0 & \gamma k_{-} & \varepsilon-\xi k^{2} & 0 \\
\gamma k_{+} & 0 & 0 & \varepsilon-\xi k^{2}
\end{array}\right) .
$$

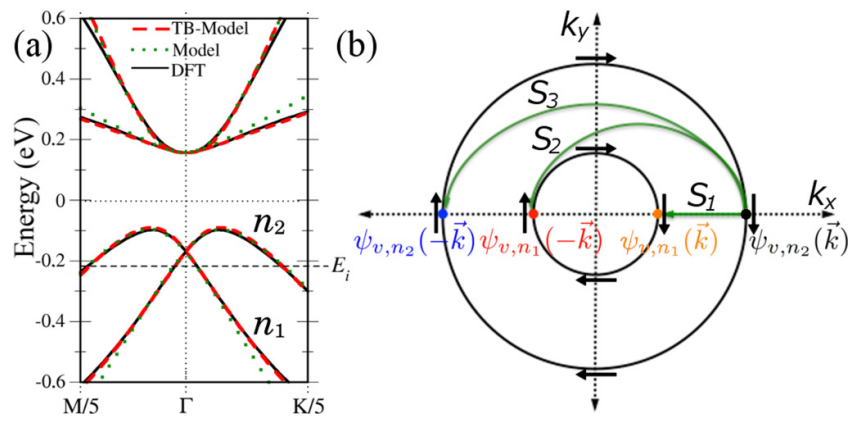

FIG. 2. (a) Band structure calculated with DFT, complete tightbinding model and simplified model. (b) Fermi contour at the energy plane $E_{i}$ obtained from the simplified model. The backscattering processes and the spin textures are represented by green and black arrows, respectively. 
We plot the Fermi contours obtained from this Hamiltonian and represent the backscattering processes in Fig. 2(b). In the valence band, an energy plane below (above) the band crossing consists of two concentric circles with the same (opposite) inplane helical spin texture. Likewise, in the conduction band, an energy plane consists of two concentric circles with the same in-plane helical spin texture. Because of this bulk spin texture, the elastic and inelastic backscattering processes represented by $S_{2}$ and $S_{3}$, respectively, are forbidden.

In order to quantify the probability of backscattering, we calculate the scattering rate due to a single Coulomb impurity considering the bare Coulomb potential [42], $\mathcal{S}_{\vec{k}^{\prime} n^{\prime}, \vec{k} n}=\frac{2 \pi}{\hbar} \frac{q_{e}^{4}}{4 A^{2} \kappa^{2} \beta^{2}}\left(1-\cos \theta_{\vec{k}^{\prime}, \vec{k}}\right) \boldsymbol{I}_{\vec{k}^{\prime}, n^{\prime}, \vec{k}, n} \delta\left(E_{n}-E_{n^{\prime}}\right)$. Here, $A$ is the unit area, $q_{e}$ is the single-electron charge, $\kappa$ is the static dielectric constant, $\beta=\left|\vec{k}-\vec{k}^{\prime}\right|$, and $I_{\vec{k}^{\prime}, n_{1}, \vec{k}, n_{2}}=$ $\left|\left\langle\psi_{E_{n_{1}}}\left(\vec{k}^{\prime}\right) \mid \psi_{E_{n_{2}}}(\vec{k})\right\rangle\right|^{2}$ is the overlap integral, which is calculated using the normalized wave function,

$$
\psi_{E_{n_{\lambda}}}(\vec{k})=\sqrt{N}\left(\begin{array}{c}
1 \\
-i \frac{k_{+}}{\alpha_{R_{1}} k^{2}} \frac{\left(\varepsilon-\xi k^{2}\right)^{2}-E_{n_{\lambda}}^{2}+\gamma^{2} k^{2}}{\varepsilon-\xi k^{2}-E_{n_{\lambda}}} \\
i \frac{\gamma}{\alpha_{R_{1}}} \frac{\left(\varepsilon-\xi k^{2}\right)^{2}-E_{n_{\lambda}}^{2}+\gamma^{2} k^{2}}{\left(\varepsilon-\xi k^{2}-E_{n_{\lambda}}\right)^{2}} \\
\frac{-\gamma k_{+}}{\left(\varepsilon-\xi k^{2}-E_{n_{\lambda}}\right)}
\end{array}\right),
$$

where $N=\frac{\left|\varepsilon-\xi k^{2}-E_{n_{\lambda}}\right|^{2}}{2\left[\left(\varepsilon-\xi k^{2}-E_{n_{\lambda}}\right)^{2}+\alpha_{\text {int }} k^{2}\right]}$. We verify that $I_{-\vec{k}^{\prime}, n_{1}, \vec{k}, n_{2}}=$ $I_{-\vec{k}, n_{\lambda}, \vec{k}, n_{\lambda}}=0$ and therefore the scattering rates $\mathcal{S}_{-\vec{k}^{\prime}, n_{1}, \vec{k}, n_{2}}$ and $\mathcal{S}_{-\vec{k}, n_{\lambda}, \vec{k}, n_{\lambda}}$ are null, proving that backscattering processes are unlikely. On the other hand, in an ordinary out-plane spin-polarized Rashba material, such as the thin films of the BiTeI 3D topological insulator [28], in an energy plane below (above) the band crossing the bands have the opposite (same) in-plane chiral spin texture [Fig. 3(a)] and therefore the elastic backscattering $S_{3}$ is forbidden. Unlike the PbBiI, in a Rashba semiconductor, below the band crossing the inelastic backscattering $S_{2}$ is allowed and $I_{-\vec{k}^{\prime}, n_{1}, \vec{k}, n_{2}} \approx 1[17,43]$, as represented by the green arrows in Fig. 3(a).

Far from the $\Gamma$ point, the $R_{3}$ symmetry breaking generates nonlinear terms in the SOC such as the three-order Rashba term $h_{R_{3}}(\vec{k})$, which induces $\left\langle S_{z}\right\rangle$ spin polarization and hexagonal warping effect in the bulk states $[44,45]$, as represented in Fig. 3(b). Although $S_{z}$ spin polarized increases, the expected value of $S_{z}$ is still near zero. Because the $\left\langle S_{x}\right\rangle$ and $\left\langle S_{y}\right\rangle$ spin flips are required so that the inelastic scattering process $\mathcal{S}_{-\vec{k}^{\prime}, n_{1}, \vec{k}, n_{2}}$ occurs [see Fig. 3(b)], the overlap integral $I_{-\vec{k}^{\prime}, n_{1}, \vec{k}, n_{2}}$ is still small compared to the value found in an ordinary Rashba semiconductors and the probability that the scattering process $S_{2}$ occurs remains low. Analogous to the surface states of $\mathrm{Bi}_{2} \mathrm{Te}_{3}$ [45], near the $\Gamma$ point, the $\left\langle S_{z}\right\rangle$ spin polarization and the warping hexagonal tend to zero and the only contribution coming from the lack of inversion symmetry is the first-order Rashba term, $h_{R_{1}}(\vec{k})$. Thus, to obtain the unconventional spin texture near the $\Gamma$ point is only enough to consider both Rashba effect and band inversion, as was done in Eq. (3). According to our DFT results, the Rashba spin splitting is about $60 \mathrm{meV}$, which is huge compared with the values found in semiconductors and surprisingly is among the highest found in $3 \mathrm{D}$ systems $[21-25,28]$. This value can be increased up to
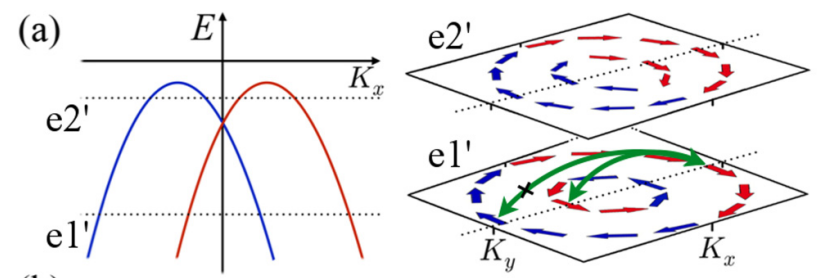

(b)
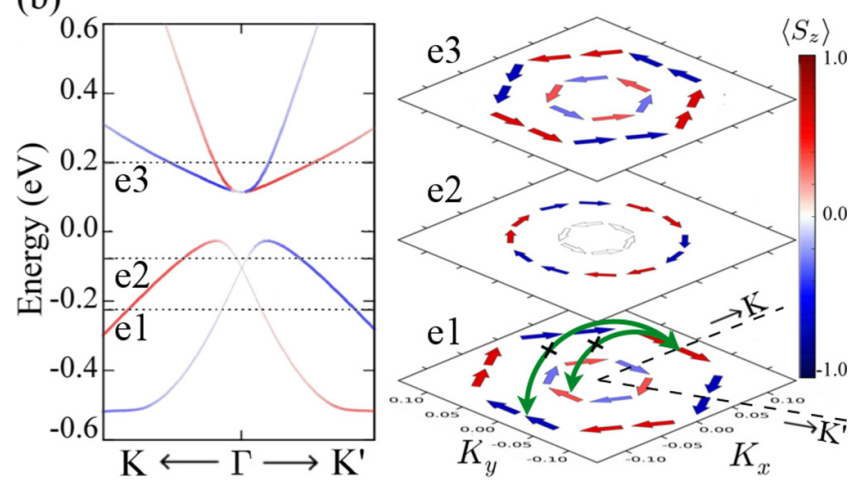

FIG. 3. Band structure and spin texture of (a) an out-plane spinpolarized Rashba material and (b) the PbBiI system. The dotted lines in the band structure represent the energy planes $\left(e 1-e 3\right.$ and $\left.e 1^{\prime}-e 2^{\prime}\right)$ in which we show the in-plane spin texture, whose magnitudes are represented by the size of the arrows. In the arrows and the band structure, the color code stands for the normalized $\left\langle S_{z}\right\rangle$ spin polarization. The backscattering processes $S_{2}$ and $S_{3}$ are represented by green arrows.

$E_{R} \approx 90 \mathrm{meV}$ by applying large compressive strain (see the Supplemental Material).

On the other hand, since the out-plane spin polarization oscillates according to the $C_{3 v}$ symmetry, as occurs in thin films of $\mathrm{Bi}_{2} \mathrm{Te}_{3}$ [45], at the $\Gamma \rightarrow M$ symmetry path, the $S_{z}$ spin component is zero (see Fig. 3) and therefore inelastic backscattering processes are completely suppressed. The armchair nanoribbon BZ is parallel to the $\Gamma \rightarrow M$ symmetry path at the $k_{y}$ axis of the hexagonal BZ. Thus, scattering processes are dominated by the $S_{x}$ spin component and hence elastic and inelastic backscattering is forbidden for bulk and edge states, as shown in Fig. 4. Similarly, the zig-zag nanoribbon BZ is parallel to the $k_{x}$ axis and therefore $\left\langle S_{x}\right\rangle=0$. Because of the nonzero $\left\langle S_{z}\right\rangle$ spin components, there is a low probability of inelastic backscattering in accordance with the bulk behavior discussed above (see the Supplemental Material). Because of the strong SOC, the spin and momentum are constrained to be perpendicular. This spin-momentum locking implies that Dirac cones of different edges are required to have the same $S_{x}$ spin texture and different $S_{z}$ spin texture of spin in the armchair nanoribbon, as represented in Fig. 4(c).

The protected bulk states near the $\Gamma$ point only appear in the energy region in which the Rashba effect and the band inversion point take place. This energy region overlaps with the bottom of the conduction band, which is at $\Gamma \rightarrow M$ symmetry path, as shown in Fig. 4(b). The bottom of the conduction band and hence the energy range in which the unusual spin texture is present can be modified by applying tensile strain (see the Supplemental Material). Indeed, the inverted band gap at $\Gamma$ can be equal to the band gap $E_{g}$ when strain is applied. 


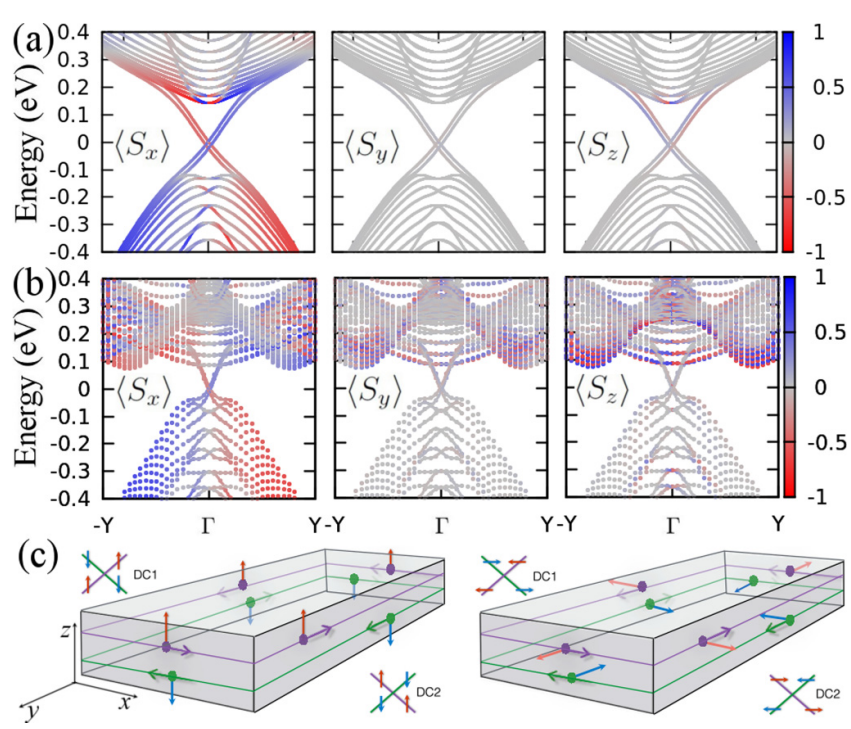

FIG. 4. (a) Tight binding and (b) DFT spin-texture band structure of an armchair nanoribbon $98.7 \AA$ wide. The color code stands for the spin polarization. (c) Pictorial representation of edge states with $\left\langle S_{z}\right\rangle$ (left) and $\left\langle S_{x}\right\rangle$ (right) spin polarization.

It is well established that the $\mathrm{Bi}-\mathrm{Pb}$ alloy can be realized experimentally by maintaining the $R \overline{3} m$ space group [46,47]. The $\mathrm{Pb}-\mathrm{Bi}$ rhombohedral alloy along the [111] direction can be considered as a stack of $\mathrm{PbBi}$ honeycomb lattices that are weakly bonded (mainly ruled by van der Waals-type interaction) to each other, similarly to the bismuth bilayers [48]. The dangling bonds that appear at the $\mathrm{Pb}$-rich $\mathrm{PbBi}$ surface can be eliminated by bonding to iodine atoms and hence the proposed spin texture could be observed in the PbBiI system via STM experiments analogously to the observation of Bi-bilayers' edge states [48].

The $C_{3 v}$ symmetry in the $\mathrm{PbBiI}$ system leads to a interaction term different from the BHZ model used to describe the QSH phase in $\mathrm{HgTe} / \mathrm{CdTe}$ quantum wells [49]. Although the $\mathrm{BHZ}$ model considering the Rashba effect has been used to describe asymmetric InAs/GaSb/AlSb quantum wells [50], the consequences of a huge Rashba spin splitting and the three-order Rashba term in a bulk inverted band gap, such as the unconventional spin texture reported here, have been ignored.

In summary, the simultaneous presence of a huge Rashba effect and an inverted band gap in systems with $C_{3 v}$ symmetry leads to conduction and valence bands with a Rashba-like spin splitting with the same helical in-plane spin texture and with null $S_{z}$ spin polarization at the $\Gamma \rightarrow M$ symmetry paths. Thus, the spin texture in the nanoribbons depends on its orientation. We find that bulk states are protected by the TR symmetry and, contrary to what happens in most doped QSH systems, the bulk states do not contribute to the backscattering, opening the way for realizing novel applications of topological edge states. Additionally, we proposed a honeycomb-lattice QSH insulator that is mechanically stable: the PbBiI system, which has a large Rashba splitting of $60 \mathrm{meV}$, a large nontrivial gap of $0.14 \mathrm{eV}$, and hence the predicted unconventional spin texture.

This work was supported by the Sao Paulo Research Foundation (Grant No. 2014/12357-3). We would like to thank Dr. Luis G. G. V. Dias da Silva for the discussions. We also acknowledge Dr. Soluyanov and Dr. Vanderbilt for sharing the code to calculate the WCCs with VASP.
[1] D. Pesin and A. H. MacDonald, Nat. Mater. 11, 409 (2012).

[2] I. Žutić, J. Fabian, and S. Das Sarma, Rev. Mod. Phys. 76, 323 (2004).

[3] A. Brataas, A. D. Kent, and H. Ohno, Nat. Mater. 11, 372 (2012).

[4] T. Liang, Q. Gibson, M. N. Ali, M. Liu, R. J. Cava, and N. P. Ong, Nat. Mater. 14, 280 (2015).

[5] L. Liu, O. J. Lee, T. J. Gudmundsen, D. C. Ralph, and R. A. Buhrman, Phys. Rev. Lett. 109, 096602 (2012).

[6] M. Z. Hasan and C. L. Kane, Rev. Mod. Phys. 82, 3045 (2010).

[7] X.-L. Qi and S.-C. Zhang, Rev. Mod. Phys. 83, 1057 (2011).

[8] C. Brune, A. Roth, H. Buhmann, E. M. Hankiewicz, L. W. Molenkamp, J. Maciejko, X.-L. Qi, and S.-C. Zhang, Nat. Phys. 8, 485 (2012).

[9] L. V. Kulik, A. V. Gorbunov, A. S. Zhuravlev, V. B. Timofeev, S. Dickmann, and I. V. Kukushkin, Sci. Rep. 5, 10354 (2015).

[10] S. Maekawa, S. O. Valenzuela, E. Saitoh, and T. Kimura, Spin Current, Series on Semiconductor Science and Technology, Vol. 17 (Oxford University Press, Oxford, UK, 2012).

[11] R. Jansen, Nat. Mater. 11, 400 (2012).

[12] A. Manchon, H. C. Koo, J. Nitta, S. M. Frolov, and R. A. Duine, Nat. Mater. 14, 871 (2015).

[13] D. Bercioux and P. Lucignano, Rep. Prog. Phys. 78, 106001 (2015).

[14] C. L. Kane and E. J. Mele, Phys. Rev. Lett. 95, 146802 (2005).
[15] M. Knig, S. Wiedmann, C. Brne, A. Roth, H. Buhmann, L. W. Molenkamp, X.-L. Qi, and S.-C. Zhang, Science 318, 766 (2007).

[16] E. B. Olshanetsky, Z. D. Kvon, G. M. Gusev, A. D. Levin, O. E. Raichev, N. N. Mikhailov, and S. A. Dvoretsky, Phys. Rev. Lett. 114, 126802 (2015).

[17] Y. A. Bychkov and E. I. Rashba, JETP Lett. 39, 78 (1984).

[18] S. LaShell, B. A. McDougall, and E. Jensen, Phys. Rev. Lett. 77, 3419 (1996)

[19] C. R. Ast, J. Henk, A. Ernst, L. Moreschini, M. C. Falub, D. Pacilé, P. Bruno, K. Kern, and M. Grioni, Phys. Rev. Lett. 98, 186807 (2007).

[20] Y. M. Koroteev, G. Bihlmayer, J. E. Gayone, E. V. Chulkov, S. Blügel, P. M. Echenique, and P. Hofmann, Phys. Rev. Lett. 93, 046403 (2004).

[21] J. Nitta, T. Akazaki, H. Takayanagi, and T. Enoki, Phys. Rev. Lett. 78, 1335 (1997).

[22] T. Hirahara, T. Nagao, I. Matsuda, G. Bihlmayer, E. V. Chulkov, Y. M. Koroteev, P. M. Echenique, M. Saito, and S. Hasegawa, Phys. Rev. Lett. 97, 146803 (2006).

[23] S. Mathias, A. Ruffing, F. Deicke, M. Wiesenmayer, I. Sakar, G. Bihlmayer, E. V. Chulkov, Y. M. Koroteev, P. M. Echenique, M. Bauer, and M. Aeschlimann, Phys. Rev. Lett. 104, 066802 (2010). 
[24] H. Yuan, M. S. Bahramy, K. Morimoto, S. Wu, K. Nomura, B.-J. Yang, H. Shimotani, R. Suzuki, M. Toh, C. Kloc, X. Xu, R. Arita, N. Nagaosa, and Y. Iwasa, Nat. Phys. 9, 563 (2013).

[25] J. H. Dil, F. Meier, J. Lobo-Checa, L. Patthey, G. Bihlmayer, and J. Osterwalder, Phys. Rev. Lett. 101, 266802 (2008).

[26] Z.-H. Zhu, G. Levy, B. Ludbrook, C. N. Veenstra, J. A. Rosen, R. Comin, D. Wong, P. Dosanjh, A. Ubaldini, P. Syers, N. P. Butch, J. Paglione, I. S. Elfimov, and A. Damascelli, Phys. Rev. Lett. 107, 186405 (2011).

[27] Y.Zhang, K. He, C.-Z. Chang, C.-L. Song, L.-L. Wang, X. Chen, J.-F. Jia, Z. Fang, X. Dai, W.-Y. Shan, S.-Q. Shen, Q. Niu, X.-L. Qi, S.-C. Zhang, X.-C. Ma, and Q.-K. Xue, Nat. Phys. 6, 584 (2010).

[28] K. Ishizaka, M. S. Bahramy, H. Murakawa, M. Sakano, T. Shimojima, T. Sonobe, K. Koizumi, S. Shin, H. Miyahara, A. Kimura, K. Miyamoto, T. Okuda, H. Namatame, M. Taniguchi, R. Arita, N. Nagaosa, K. Kobayashi, Y. Murakami, R. Kumai, Y. Kaneko, Y. Onose, and Y. Tokura, Nat. Mater. 10, 521 (2011).

[29] M. S. Bahramy, B. J. Yang, R. Arita, and N. Nagaosa, Nat. Commun. 3, 679 (2012).

[30] T. Das and A. V. Balatsky, Nat. Commun. 4, 1972 (2013).

[31] J.-J. Zhou, W. Feng, Y. Zhang, S. A. Yang, and Y. Yao, Sci. Rep. 4, 3841 (2014).

[32] J. M. Soler, E. Artacho, J. D. Gale, A. García, J. Junquera, P. Ordejón, and D. Sánchez-Portal, J. Phys. Condens. Matter 14, 2745 (2002).

[33] G. Kresse and J. Furthmüller, Phys. Rev. B 54, 11169 (1996).

[34] C. M. Acosta, M. P. Lima, R. H. Miwa, A. J. R. da Silva, and A. Fazzio, Phys. Rev. B 89, 155438 (2014).

[35] L. Fernandez-Seivane, M. A. Oliveira, S. Sanvito, and J. Ferrer, J. Phys. Condens. Matter 18, 7999 (2006).

[36] J. P. Perdew and A. Zunger, Phys. Rev. B 23, 5048 (1981).
[37] J. P. Perdew, K. Burke, and M. Ernzerhof, Phys. Rev. Lett. 77, 3865 (1996).

[38] See Supplemental Material at http://link.aps.org/supplemental/ 10.1103/PhysRevB.94.041302 for details on the phonon spectrum calculation, band gap and Rasha splitting behavior as a function of the lattice constant, deduction of the model, scattering rate calculations and the evolution of Wannier Center of Charges.

[39] R. Yu, X. L. Qi, A. Bernevig, Z. Fang, and X. Dai, Phys. Rev. B 84, 075119 (2011).

[40] A. A. Soluyanov and D. Vanderbilt, Phys. Rev. B 83, 035108 (2011).

[41] A. A. Soluyanov and D. Vanderbilt, Phys. Rev. B 83, 235401 (2011).

[42] G. Yin, D. Wickramaratne, Y. Zhao, and R. K. Lake, Appl. Phys. Lett. 105, 033118 (2014).

[43] K. Sakamoto, T.-H. Kim, T. Kuzumaki, B. Müller, Y. Yamamoto, M. Ohtaka, J. R. Osiecki, K. Miyamoto, Y. Takeichi, A. Harasawa, S. D. Stolwijk, A. B. Schmidt, J. Fujii, R. I. G. Uhrberg, M. Donath, H. W. Yeom, and T. Oda, Nat. Commun. 4, 2073 (2013).

[44] S. Vajna, E. Simon, A. Szilva, K. Palotas, B. Ujfalussy, and L. Szunyogh, Phys. Rev. B 85, 075404 (2012).

[45] L. Fu, Phys. Rev. Lett. 103, 266801 (2009).

[46] N. Gokcen, J. Phase Equilib. 13, 21 (1992).

[47] H. C. W. Huang and C. M. Serrano, J. Vac. Sci. Technol. A 1, 1409 (1983).

[48] I. K. Drozdov, A. Alexandradinata, S. Jeon, S. Nadj-Perge, H. Ji, R. J. Cava, B. Andrei Bernevig, and A. Yazdani, Nat. Phys. 10, 664 (2014).

[49] B. A. Bernevig, T. L. Hughes, and S.-C. Zhang, Science 314, 1757 (2006).

[50] C. Liu, T. L. Hughes, X.-L. Qi, K. Wang, and S.-C. Zhang, Phys. Rev. Lett. 100, 236601 (2008). 


\section{Bibliografia}

[1] S. A. Wolf, D. D. Awschalom, R. A. Buhrman, J. M. Daughton, S. Von Molnár, M. L. Roukes, A. Y. Chtchelkanova, D. M. Treger, Spintronics: A Spin-Based Electronics Vision for the Future, Science v294 55461488 (2001)

[2] Pesin, Dmytro and MacDonald, Allan H, Spintronics and pseudospintronics in graphene and topological insulators, Nat Mater, 115 409-416 (2012)

[3] Igor and Fabian, Jaroslav and Das Sarma, S, Spintronics: Fundamentals and applications, Rev. Mod. Phys, 762 323-410 (2004)

[4] R. Winkler, Spin-Dependent Transport of Carriers in Semiconductors (Handbook of Magnetism and Advanced Magnetic Materials). John Wiley Son, New York, 2007.

[5] J. Fabian, A. Matos-Abiague, C. Ertler, P. Stano, I. Zutic, Semiconductor Spintronics, Acta Phys. Slovaca 57, 565-907 (2007)

[6] Min, Hongki and Hill, J. E. and Sinitsyn, N. A. and Sahu, B. R. and Kleinman, Leonard and MacDonald, A. H., Intrinsic and Rashba spin-orbit interactions in graphene sheets Phys. Rev. B, 7416165310 (2006)

[7] Hirahara, T. and Nagao, T. and Matsuda, I. and Bihlmayer, G. and Chulkov, E. V. and Koroteev, Yu. M. and Echenique, P. M. and Saito, M. and Hasegawa, 
S., Role of Spin-Orbit Coupling and Hybridization Effects in the Electronic Structure of Ultrathin Bi Films, Phys. Rev. Lett., 9714146803 (2006)

[8] Bernevig, B. Andrei and Hughes, Taylor L. and Zhang, Shou-Cheng, Quantum Spin Hall Effect and Topological Phase Transition in HgTe Quantum Wells, Science, 3145806 1757-1761 (2006)

[9] Nitta, Junsaku and Akazaki, Tatsushi and Takayanagi, Hideaki and Enoki, Takatomo, Gate Control of Spin-Orbit Interaction in an Inverted $\operatorname{In}_{0.53} G \mathrm{a}_{0.47} A s / \mathrm{In}_{0.52} A \mathrm{l}_{0.48}$ As Heterostructure, Phys. Rev. Lett., 787 1335-1338 (1997)

[10] Kulik, L. V. and Gorbunov, A. V. and Zhuravlev, A. S. and Timofeev, V. B. and Dickmann, S. and Kukushkin, I. V, Super-long life time for $2 D$ cyclotron spin-flip excitons, Sci. Rep, 5 pg 10354 (2015)

[11] Y. A. Bychkov and E. I. Rashba, Oscillatory effects and the magnetic susceptibility of carriers in inversion layers J. Phys. C 17, 6039 (1984).

[12] Bychkov, Y. A. and Rashba, E. I, Properties of a $2 D$ electron gas with lifted spectral degeneracy, JETP Lett, 39278 (1984)

[13] Born, M. Oppenheimer, R. Zur quantentheorie der molekeln. Ann. Phys. (Leipzig), v84 20457 (1927)

[14] Hohenberg, P. Kohn, W. Inhomogeneous electron gas. Physical Review, v136 B864, (1964)

[15] Thomas,L. H. "The calculation of atomic fields". Mathematical Proceedings of the Cambridge Philosophical Society. v23 05 542-548 (1927)

[16] Fermi, E. "Statistische Berechnung der Rydbergkorrektionen ders-Terme". Zeitschrift für Physik 49, 550-554 (1928) 
[17] Dirac,P. A. M, "Note on Exchange Phenomena in the Thomas Atom". Mathematical Proceedings of the Cambridge Philosophical Society, v26 03 376-385 (1930)

[18] Kohn, W. Sham, L. Self-consistent equations including exchange and correlation effects. Physical Review A, v140 1133, (1965)

[19] J.P. Perdew and Y. Wang, Accurate and simple density functional for the electronic exchange energy: Generalized gradient approximation. Phys. Rev. B $33,8800(1986)$

[20] D. M. Ceperley and B. J. Alder, "Ground state of the electron gas by a stochastic method" Phys. Rev. Lett., v45 566 (1980).

[21] J. P. Perdew and Alex Zunger, "Self-interaction correction to density-functional approximations for many-electron systems". Phys. Rev. B $23,5048(1981)$

[22] Perdew, J. P.; Burke, K.; Ernzenhof, M. Generalized gradient approximation made simple. Physical Review Letters, v77 3865 (1996)

[23] H. Zhang, Chao-Xing Liu, Xiao-Liang, Xi Dai, Z. Fang, S. C. Zhang. Topological insulators in Bi2Se3, Bi2Te3 and Sb2Te3 with a single Dirac cone on the surface, Nature Physics 5, 438 - 442 (2009).

[24] P. A. M. Dirac, "The Quantum Theory of the Electron" Proceedings of the Royal Society of London. Series A, Containing Papers of a Mathematical and Physical Character, Volume 117, Issue 778, pp. 610-624 (1928)

[25] Thomas, L. H. "Motion of the spinning electron". Nature 117: 514. (1926)

[26] K.V. Samokhin, Spin-orbit coupling and semiclassical electron dynamics in noncentrosymmetric metals, Ann. Phys. (N.Y.) 324112385 (2009) 
[27] G. Dresselhaus, Spin-Orbit Coupling Effects in Zinc Blende Structures, Phys. Rev. 100, 580 (1955)

[28] S. D. Kevan and R. H. Gaylord, High-resolution photoemission study of the electronic structure of the noble-metal (111) surfaces, Phys Rev B 365809 (1987)

[29] Sz. Vajna, E. Simon, A. Szilva, K. Palotas, B. Ujfalussy, and L. Szunyogh, Higher-order contributions to the Rashba-Bychkov effect with application to the Bi/Ag(111) surface alloy. Phys. Rev. B 85, 075404 (2012)

[30] Ast, Christian R. and Henk, Jürgen and Ernst, Arthur and Moreschini, Luca and Falub, Mihaela C. and Pacilé, Daniela and Bruno, Patrick and Kern, Klaus and Grioni, Marco, Giant Spin Splitting through Surface Alloying, Phys. Rev. Lett., 9818186807 (2007)

[31] Emmanouil Frantzeskakis, Stéphane Pons, Hossein Mirhosseini, Jürgen Henk, Christian R. Ast, and Marco Grioni, Tunable Spin Gaps in a Quantum-Confined Geometry, Phys. Rev. Lett. 101, 196805 (2008)

[32] Murakami, S., Nagaosa, N., and Zhang S.C., Dissipationless Quantum Spin Current at Room Temperature, Science, 301, 1348 (2003)

[33] Sinova, J., Culcer, D., Niu, Q., Sinitsyn, N. A., Jungwirth, T., and Mac-Donald, A. H., Universal Intrinsic Spin Hall Effect, Phys. Rev. Lett. 92, $126603(2004)$

[34] Sadamichi Maekawa and Sergio O. Valenzuela and Eiji Saitoh and Takashi Kimura, Spin Current, (2012)

[35] M. I. Dyakonov and V. I. Perel', "Current-induced spin orientation of electrons in semiconductors". Phys. Lett. A 35, 459 (1971) 
[36] Fu, Liang and Kane, C. L., Time reversal polarization and a $\mathbb{Z}_{2}$ adiabatic spin pump, Phys. Rev. B, 7419195312 (2006)

[37] Yu, Rui and Qi, Xiao Liang and Bernevig, Andrei and Fang, Zhong and Dai, $\mathrm{Xi}$, Equivalent expression of $\mathbb{Z}_{2}$ topological invariant for band insulators using the non-Abelian Berry connection. Phys. Rev. B, 847075119 (2011)

[38] Kane, C. L. and Mele, E. J., $\mathbb{Z}_{2}$ Topological Order and the Quantum Spin Hall Effect, Phys. Rev. Lett., 9514146802 (2005)

[39] Liang Fu and C. L. Kane, Topological insulators with inversion symmetry, PRB 76,045302 (2007)

[40] M. Z. Hasan, Observation of a large-gap topological-insulator class with a single Dirac cone on the surface, Nature Physics 5, 398 - 402 (2009) .

[41] L. Seixas, L. B. Abdalla, T. M. Schmidt, A. Fazzio1 and R. H. Miwa, Topological states ruled by stacking faults in $B i_{2} \mathrm{Se}_{3}$ and $B i_{2} \mathrm{Te}_{3}$,J. Appl. Phys. 113, $023705(2013)$

[42] C. Mera Acosta, Matheus P. Lima, Antônio J. R. da Silva, C. H. Lewenkopf, and A. Fazzio. Microscopic origin of bulk-states near the Dirac-cone in rhombohedrical topological insulators. (sumited).

[43] O Jepsen, Electronic structure and thermoelectric properties of bismuth telluride and bismuth selenide, J. Phys. Condens. Matter 9461 (1997)

[44] Leonardo Batoni Abdalla, "Propriedades eletrônicas dos Isolantes Topológico". Tese de doutorado, Instituto de Física, Universidade de São Paulo (2015)

[45] Yoichi Ando, Topological Insulator Materials, J. Phys. Soc. Jpn. 82, 102001 $(2013)$ 
[46] Chao-Xing Liu, Xiao-Liang Qi, HaiJun Zhang, Xi Dai, Zhong Fang, and Shou-Cheng Zhang. Model Hamiltonian for topological insulators, PHYSICAL REVIEW B 82, 045122 (2010)

[47] Kazuto Ebihara, Keiji Yada, Ai Yamakage, Yukio Tanaka. Finite size effects of the surface states in a lattice model of topological insulator. Physica E 44 (2012) 885-890

[48] Hor, Y. S. and Roushan, P. and Beidenkopf, H. and Seo, J. and Qu, D. and Checkelsky, J. G. and Wray, L. A. and Hsieh, D. and Xia, Y. and Xu, S.-Y. and Qian, D. and Hasan, M. Z. and Ong, N. P. and Yazdani, A. and Cava, R. J., Development of ferromagnetism in the doped topological insulator $\mathrm{Bi}_{2-x} \mathrm{Mn}_{x} \mathrm{Te}_{3}$, Phys. Rev. B, 8119195203 (2010)

[49] Rui Yu1, Wei Zhang, Hai-Jun Zhang, Shou-Cheng Zhang, Xi Dai, Zhong Fang, Quantized Anomalous Hall Effect in Magnetic Topological Insulators, Science 329, 61 (2010)

[50] Kriener, M. and Segawa, Kouji and Ren, Zhi and Sasaki, Satoshi and Ando, Yoichi, Bulk Superconducting Phase with a Full Energy Gap in the Doped Topological Insulator $\mathrm{Cu}_{x} \mathrm{Bi}_{2} \mathrm{Se}_{3}$, Phys. Rev. Lett. 106, 127004 (2011)

[51] Hor, Y. S. and Williams, A. J. and Checkelsky, J. G. and Roushan, P. and Seo, J. and Xu, Q. and Zandbergen, H. W. and Yazdani, A. and Ong, N. P. and Cava, R. J. Superconductivity in $\mathrm{Cu}_{x} \mathrm{Bi}_{2} \mathrm{Se}_{3}$ and its Implications for Pairing in the Undoped Topological Insulator, Phys. Rev. Lett. 104, 057001 (2010)

[52] Yang, Fan and Ding, Yue and Qu, Fanming and Shen, Jie and Chen, Jun and Wei, Zhongchao and Ji, Zhongqing and Liu, Guangtong and Fan, Jie and Yang, Changli and Xiang, Tao and Lu, Li, Proximity effect at superconducting $S n-B i_{2} S_{3}$ interface, Phys. Rev. B 85104508 (2012) 
[53] Feng-feng Zhu, Wei-jiong Chen, Yong Xu, Chun-lei Gao, Dan-dan Guan, Can-hua Liu, Dong Qian, Shou-Cheng Zhang and Jin-feng Jia, Epitaxial growth of two-dimensional stanene, Nature Materials 14, 1020-1025 (2015)

[54] Acosta, C. Mera and Lima, Matheus P. and Miwa, R. H. and da Silva, Antônio J. R. and Fazzio, A., Topological phases in triangular lattices of Ru adsorbed on graphene: Ab initio calculations, Phys. Rev. B, 8915155438 (2014)

[55] Fernández-Seivane, Lucas and Oliveira, Miguel A and Sanvito, Stefano and Ferrer, Jaime, On-site approximation for spin-orbit coupling in linear combination of atomic orbitals density functional methods, J. of Phys.: Condens. Matter, 18347999 (2006)

[56] Guanhua Zhang, Huajun Qin, Jing Teng, Jiandong Guo, Qinlin Guo, Xi Dai, Zhong Fang and Kehui Wu, Quintuple-layer epitaxy of thin films of topological insulator $\mathrm{Bi}_{2} \mathrm{Se}_{3}$, Appl. Phys. Lett. 95, 053114 (2009)

[57] Fu, Liang and Kane, C. L., Topological insulators with inversion symmetry, Phys. Rev. B, 764045302 (2007)

[58] Lee, C.; Yang, W.; Parr, R. G. Development of the colle-salvetti correlation energy formula into a functional of the electron density. Physical Review B, v. 37, p. 785, 1988.

[59] Becke, D. Density-functional thermochemistry. iii. the role of exact exchange. Journal Chemical Phys, v. 98, p. 5648, (1993)

[60] W. Gerlach, O. Stern, Der experimentelle Nachweis des magnetischen Moments des Silberatoms, Zeitschrift für Physik, Volume 9, Issue 1, pp.349-352

[61] Shijun Mao, Ai Yamakage, and Yoshio Kuramoto, Tight-binding model for topological insulators: Analysis of helical surface modes over the whole Brillouin zone, Phys. Rev. B 84, 115413 (2011) 
[62] Liang Fu, C. L. Kane, and E. J. Mele, Topological Insulators in Three Dimensions, Phys Rev Lett 98, 106803 (2007)

[63] J. E. Moore and L. Balents, Topological invariants of time-reversal-invariant band structures, Phys Rev B 75, 121306 (2007)

[64] Rahul Roy, Z2 classification of quantum spin Hall systems: An approach using time-reversal invariance, Phys. Rev. B 79, 195321 (2009)

[65] Liang, Tian and Gibson, Quinn and Ali, Mazhar N. and Liu, Minhao and Cava, R. J. and Ong, N. P, Ultrahigh mobility and giant magnetoresistance in the Dirac semimetal Cd3As2, Nat Mater, 143 280-284 (2015),

[66] Liu, Luqiao and Lee, O. J. and Gudmundsen, T. J. and Ralph, D. C. and Buhrman, R. A, Current-Induced Switching of Perpendicularly Magnetized Magnetic Layers Using Spin Torque from the Spin Hall Effect, Phys. Rev. Lett, 1099096602 (2012),

[67] Brataas, Arne and Kent, Andrew D. and Ohno, Hideo, Current-induced torques in magnetic materials, Nat Mater, 115 372-381 (2012)

[68] Qi, Xiao-Liang and Zhang, Shou-Cheng, Topological insulators and superconductors, Rev. Mod. Phys, 834 1057-1110 (2011)

[69] Hasan, M. Z. and Kane, C. L, Colloquium : Topological insulators, Rev. Mod. Phys, 824 3045-3067 (2010)

[70] Jansen, Ron, Silicon spintronics, Nat Mater, 115 400-408 (2012)

[71] Manchon, A. and Koo, H. C. and Nitta, J. and Frolov, S. M. and Duine, R. A., New perspectives for Rashba spin-orbit coupling, Nat Mater, 149 871-882 (2015) 
[72] Dario Bercioux and Procolo Lucignano, Quantum transport in Rashba spin-orbit materials: a review, Reports on Progress in Physics, 7810106001 $(2015)$

[73] Ishizaka K. and Bahramy M. S. and Murakawa H. and Sakano M. and Shimojima T. and Sonobe T. and Koizumi K. and Shin S. and Miyahara H. and Kimura A. and Miyamoto K. and Okuda T. and Namatame H. and Taniguchi M. and Arita R. and Nagaosa N. and Kobayashi K. and Murakami Y. and Kumai R. and Kaneko Y. and Onose Y. and Tokura Y., Giant Rashba-type spin splitting in bulk BiTeI, Nat Mater, 107 521-526 (2011)

[74] Zhu, Z.-H. and Levy, G. and Ludbrook, B. and Veenstra, C. N. and Rosen, J. A. and Comin, R. and Wong, D. and Dosanjh, P. and Ubaldini, A. and Syers, P. and Butch, N. P. and Paglione, J. and Elfimov, I. S. and Damascelli, A., Rashba Spin-Splitting Control at the Surface of the Topological Insulator $\mathrm{Bi}_{2} \mathrm{Se}_{3}$, Phys. Rev. Lett.,107 18186405 (2011)

[75] Zhang, Yi and He, Ke and Chang, Cui-Zu and Song, Can-Li and Wang, Li-Li and Chen, Xi and Jia, Jin-Feng and Fang, Zhong and Dai, Xi and Shan, Wen-Yu and Shen, Shun-Qing and Niu, Qian and Qi, Xiao-Liang and Zhang, Shou-Cheng and Ma, Xu-Cun and Xue, Qi-Kun, Crossover of the three-dimensional topological insulator Bi2Se3 to the two-dimensional limit Nat Phys, 68 584-588 (2010)

[76] Bahramy, M. S. and Yang, B. -J. and Arita, R. and Nagaosa, N., Emergence of non-centrosymmetric topological insulating phase in BiTeI under pressure, Nat Commun, 3679 (2012)

[77] Das, Tanmoy and Balatsky, A. V., Engineering three-dimensional topological insulators in Rashba-type spin-orbit coupled heterostructures, Nat Commun, 4 $1972(2013)$ 
[78] Zhou, Jin-Jian and Feng, Wanxiang and Zhang, Ying and Yang, Shengyuan A. and Yao, Yugui, Engineering Topological Surface States and Giant Rashba Spin Splitting in BiTeI/Bi2Te3 Heterostructures, Scientific Reports, 43841 (2014)

[79] Soler, José M and Artacho, Emilio and Gale, Julian D and García, Alberto and Junquera, Javier and Ordejón, Pablo and Sánchez-Portal, Daniel, The SIESTA method for ab initio order- $N$ materials simulation, Journal of Physics: Condensed Matter, 14112745 (2002)

[80] Kresse, G. and Furthmüller, J., Efficient iterative schemes for ab initio total-energy calculations using a plane-wave basis set, Phys. Rev. B, 5416 11169-11186 (1996)

[81] Perdew, John P and Zunger, Alex, Self-interaction correction to density-functional approximations for many-electron systems, Phys. Rev. B, 23 105048 (1981)

[82] Perdew, John P. and Burke, Kieron and Ernzerhof, Matthias, Generalized Gradient Approximation Made Simple, Phys. Rev. Lett., 7718 3865-3868 (1996)

[83] Mathias, S. and Ruffing, A. and Deicke, F. and Wiesenmayer, M. and Sakar, I. and Bihlmayer, G. and Chulkov, E. V. and Koroteev, Yu. M. and Echenique, P. M. and Bauer, M. and Aeschlimann, M., Quantum-Well-Induced Giant Spin-Orbit Splitting, Phys. Rev. Lett., 1046066802 (2010)

[84] Yuan, Hongtao and Bahramy, Mohammad Saeed and Morimoto, Kazuhiro and Wu, Sanfeng and Nomura, Kentaro and Yang, Bohm-Jung and Shimotani, Hidekazu and Suzuki, Ryuji and Toh, Minglin and Kloc, Christian and Xu, Xiaodong and Arita, Ryotaro and Nagaosa, Naoto and Iwasa, Yoshihiro, Zeeman-type spin splitting controlled by an electric field, Nat Phys, 99 563-569 (2013) 
[85] Dil, J. Hugo and Meier, Fabian and Lobo-Checa, Jorge and Patthey, Luc and Bihlmayer, Gustav and Osterwalder, Jürg, Rashba-Type Spin-Orbit Splitting of Quantum Well States in Ultrathin Pb Films, Phys. Rev. Lett., 10126266802 (2008)

[86] Sakamoto, Kazuyuki and Kim, Tae-Hwan and Kuzumaki, Takuya and Müller, Beate and Yamamoto, Yuta and Ohtaka, Minoru and Osiecki, Jacek R. and Miyamoto, Koji and Takeichi, Yasuo and Harasawa, Ayumi and Stolwijk, Sebastian D. and Schmidt, Anke B. and Fujii, Jun and Uhrberg, R. I. G. and Donath, Markus and Yeom, Han Woong and Oda, Tatsuki, Valley spin polarization by using the extraordinary Rashba effect on silicon, Nat Commun, $42073(2013)$

[87] Fu, Liang, Hexagonal Warping Effects in the Surface States of the Topological Insulator $\mathrm{Bi}_{2} \mathrm{Te}_{3}$, Phys. Rev. Lett., 10326266801 (2009)

[88] Vajna, Sz. and Simon, E. and Szilva, A. and Palotas, K. and Ujfalussy, B. and Szunyogh, L., Higher-order contributions to the Rashba-Bychkov effect with application to the Bi/Ag(111) surface alloy, Phys. Rev. B, 857075404 (2012)

[89] Liu, Chaoxing and Hughes, Taylor L. and Qi, Xiao-Liang and Wang, Kang and Zhang, Shou-Cheng, Quantum Spin Hall Effect in Inverted Type-II Semiconductors, Phys. Rev. Lett., 10023236601 (2008) 